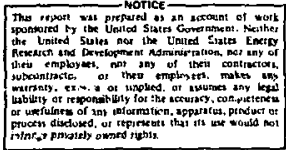

Rem tene; verba sequentur.

Cato, The Elder

Grasp the subject, the words will follow. 
TABLE OF JONTENTS

Abstract

I. Introduction

II. PAC Formalism

A. The Unperturbed Directional Correlation Function . . . 5

P. The Perturbed Directional Correlation Function for

Static Interactions . . . . . . . . . . . 7

C. Applied Magnetic Fields . . . . . . . . . . . 10

III. Apparatus. . . . . . . . . . . . . . . . . 11

A. The Four-Counter System Electronics . . . . . . . . 11

B. The Eight-Counter System Electronics. . . . . . . . 13

C. The High-Pressure Cell, Tenperature Regulator,

and Miscellaneous Equipment . . . . . . . . 15

IV. Data Analysis. . . . . . . . . . . . . . . . . 21

A. Extraction of the Factor $\mathrm{A}_{22} \mathrm{G}_{22}(\mathrm{t})$. . . . . . . . 21

B. Least Squares Fitting of $\bar{A}_{22} \mathrm{G}_{22}(\mathrm{t})$. . . . . . . . 22

Acknowledgments . . . . . . . . . . . . . . . 25

V. Systematics of Hyperfine Fields Observed by PAC in

Cd-Doped Transition Metal Antiferromagnets . . . . . 28

A. Introduction . . . . . . . . . . . . . 28

B. Deteztor System . . . . . . . . . . . . . 29

C. LCAO-MD Model . . . . . . . . . . . . . . . 36

D. Systematics of the Cd Hyperfine Fields and

Spin Density Parameters . . . . . . . . . 53

E. Conclusion . . . . . . . . . . . . . . 62

References . . . . . . . . . . . . . . . . 63 
VI. Measurements of Solid State Phenomena . . . . . . . . 67

A. Measurement of Spin Deviations, Pressure Induced Hyperfine

Field Shifts; and Sublattice Magnetizations Using

Perturbed Angular Correlations . . . . . . . 67

Acknowledgments . . . . . . . . . . . . . . . . . 83

References . . . . . . . . . . . . . . . . 84

B. F ramagnetic Shifts and Spin-Flop in Supertransferred

Hyperfine Structure of ${ }^{111 \mathrm{~m}} \mathrm{Cd}$ in $\mathrm{RbMhF}_{3}$. . . . . 87

References . . . . . . . . . . . . . . . . . 92

VII. Rare-Earth Valence State Studies of the Series

$\mathrm{RIn}_{3}$ and $\mathrm{KSn}_{3}$ Derived from Quadrupole Coupling

Constants . . . . . . . . . . . . . . . 94

A. Introduction . . . . . . . . . . . . . . . 94

B. Experimental . . . . . . . . . . . . . 96

C. Results . . . . . . . . . . . . . . . . 98

D. Interpretation . . . . . . . . . . . . . . 103

Acknowledgments . . . . . . . . . . . . . . . . 112

References . . . . . . . . . . . . . . . . 113 


\title{
APPLICATIONS OF TIME-DIFFERENTIAL PERTURBED
}

ANGULAR CORRELATIONS TO THE STUDY OF SOLIDS

Gary Paul Schwartz

(Ph. D. Thesis)

Department of Chemistry, and Lawrence Berkeley Laboratory University of California, Berkeley, Cúlifornia 94720

\author{
Apri1 1975
}

\section{ABSTRACT}

Time-differential perturbed angular correlation techniques were applied to a systematic study of insulating antiferromagnets and rareearth intermetallic alloys doped with either ${ }^{111} \mathrm{~m}$ Cd or ${ }^{111_{1 n}}$. The internal magnetic fields and electric field gradients at the radioactive nucleus are deduced from the cyperimentally measured perturbation factors. The analysis of fluoride, chloride, oxide, and sulfide data shows the systematic variation of the observed supertransferred hyperfine fields with the intervening anion covalency and allows one to extract covalency parameters after the adoption of a simple model. A comparison of the transferred hyperfine field data between fluoride perovskites and the $\mathrm{CO}^{\mathrm{i}}$ responding quadratic layer compounds produces a value for the zero-point spin deviation in magnetically two-dimensional antiferromagnets which is in qualitative agreement with existing theoretical estimates. Paramagnetic shifts due to transferred hyperfine field and field-induced spin-flopping have also been observed. By careful temperature regulation we have been able to plot out the temperature dependence of the sublattice magnetization next to a diamagne:ic impurity in $\mathrm{RbMnF}_{3}$ and $\mathrm{MnF}_{2}$. A shift in the 


\section{-vi-}

transferred hyperfine field at $\mathrm{Cd}$ doped into $\mathrm{MnS}$ has been measured under the application of moderate pressures up to $22 \mathrm{kbar}$. Analysis of the electric field gradients at the In and $\mathrm{Sn}$ sites in the rare-earth series $\mathrm{RIn} n_{3}$ and $\mathrm{RSn}_{3}$ as functions of temperature and pressure has permitted us to check for valence fluctuations in certain of these alloys. 


\section{INTRODUCTION}

The early 20th century saw the advent of the directed valence bond proposals of pauiing ${ }^{l}$ and its associated concept of covalent bonding between atoms. It was not known to what degree the idea of covalent bonding could be applied to salts contajning strongly electronegative anions, and Van vleck ${ }^{2}$ had developed a relatively successful model to explain the magnecic properties of a number of transition metal salts based on a purely "ioniz" crystal field analysis. The postwar application of the new resonance techniques (MR, EPR, ENDOR) to these materials, previously considered to be purely ionic, was to prove a terting ground for the general applicability of the covalency concept and for the further elucidation of the fundamental nature of the chemical bond.

The nature of the chenical bond was not the only problem which drew attention to the transition metal salts; concurrent theoretical advances by Kramers ${ }^{3}$ involving the role of nomagnetic atoms in explaiining the existence of long range magnetic order had been put forth and developed subsequently by many authors. The concept of superexchange 4 associated with the change in the paranagnetic ion wave function due to admixture of syme ry allowed ligand orbitals was integrally tied to the discussion of covalenc; in these compounds.

The late entry of neutron diffraction, Aössbauer, and perturbed angular correlation studies into this field can be understood in terms of the experimental difficulties which must be sumounted. For PAC measurements the insulating nature of these compounds requires a nuclear cascade which proceeds via either beta decay or an isomeric trarsition in order to avoid multiply charged electronic staties following the decay 
which would wash out the corresation. The radioactive visleus must have in addition an ionic radius and oxidation state comensurate with that of the magnetic impurity which it replaces. These stringent requirements narrow the field of available nuclei to essentially one curdidate, ${ }^{11} \mathrm{~lm} \mathrm{Cd}$, with its attendant half-life of 49 minutes. Since $C d p$ cr d orbitals can contribute to nur.lear magnetic fields only through core folarization, transferred spin density into $C d s$ shells via the Fermi contact term will dominate the magnetic hyperfine interaction. The spherical symmetry exhibited by $s$ shells results in a $a$ or $s$ bond specific interaction, thereby providing a distinct advantage over other techniques.

It vas this combination of unique bonding information available to PAC and the continued need to study concentrated magnetic systems exhibiting long range order which provided the impetus for undertaking the measurements. We thus systematically studied a large range of insulting ant iferromagnets which encompassed , lworides, oxides, chlorides, and sulfides as aniors. In order to make comparisons between compcunds with differen. covalency parameters and to check the predictions of superexchange theories, we have further required the impurity to be octahedrally coordinated with only linear metal-1igand-impurity bonds.

our interests have ranged beyond the data available from a simple chemical bond analysis; we have also used the transferred hyperfine field at the cd nueleus in order to probe a variety of solid state phenomena. In this vein we have detected spin deviatiors in magnetically two-dimensional antiferromagnets due to quantum mechanical zero-point motion as well as probing the terperature dependence of the sublattice magnetization next to a diallignetic impurity. Measurenents in an applied 
external field have yielded paramagnetic shifts due to transferred fields and the observation of the flipping of the magnetization axis at a critical field in $\mathrm{RbNhF}_{3}$.

The quest to appiy PAC to the study of physical phenomena has not been 1 imited to utilizing transferred magnetic fields. Using the electric field gradient at the tetragonal site in the rare-earth series $\operatorname{RIn}_{3}$ and $\mathrm{RSn}_{3}$, we have demonstrated a high sensitivity method for discriminating between rare-earth valence states ani have checked for temperature and pressure induced valence fluctuations in a number of $\mathrm{Ce}$, $\mathrm{Eu}$, and $\mathrm{Yh}$ compounds. 


\section{I1. PAC FORMALISM}

The exact quantum mechanical description of the angular distribution of radiations emitted from an ensemble of nuclei is based on the transformation properties of spherical tensors, the manipularions of Racas: algebra, and the statistical formaiism of the density matrix. I plan to forego duplicating this involved description and will pursue instead the basic physical ideas involved and will describe in a general way the results which will be pertinent to the data analysis. The reader who requires more rigor will find that the iiterature $5,6,7$ fairly abounds in comprehensive tracts which include all of the formal details of the theory.

Our basic picture centers around a statistical ensemble of nuclei which will emit radiation isotropically if they have no preferred direction in space. We wish to explain in physical terms how this ensemble is prepared so that the associated nuclear angular momenta are preferentially oriented with the result that the enitted radiation will in general be anisotropic.

If one considers a deexcitation taking place between two nuclear levels with angular momentum $I_{i}$ and $I_{f}$ with projection quantum numbers $M_{i}$ and $M_{f}$, then the quantur mechanical description of the radiated angular manentum of a photon field contains in it the selection rules for allowed multipole transitions between quantum states. A photon carrying $h$ l units of angular momentum with projection quantum number $m$ connects the initial and final states such shat

$$
\begin{aligned}
& \left|I_{i}-I_{f}\right| \leqslant \ell \leqslant\left|I_{i}+I_{f}\right| \\
& m=M_{i}-M_{f} .
\end{aligned}
$$


In principal the emitted radiation will contain a11. the mulripole character consistent with the allowed values of $\ell$, but in practice the transition probabilities are scaled by a factor of order $(a / \lambda)^{2 \ell}$, where $a$ and $\lambda$ are parameters characteristic of the nuclear cisameter and the wavelength of the emitted radiation. For the actual cascades (Fig. 1) which rere used in this work, the radiations are essentially untired and carry away the mirimen alle angular momentum. By performing a measurement which detects the emitted photon, we select out of space a quantization axis with respect to the ensemble of nuclei. In the simplest case $(\ell=1$, dipole radiation), the projection of the photons angular momentum along the quantizarion axis is $m= \pm 1$. In this case the state $m=0$ is not allowed since a transverse field cannot carry angular monentum along its direction of propagation. Inspection of the selection rules (Eq. 1) for the allowed transicions shows that certain transitions are now forbidden and thus the final state subpopulations associared with the $M_{f}$ quantum numbers will be unequal. As equivalent statement is that the final state now shows a preferential direction in space specified by the quantization axis, and subsequent photon emission from this state will not be generally isotropic.

\section{A. The Unperturbed Directional Correlation Furction}

The techniques of Racah algebra and the density matrix formalisn! for handling an ensemble of statistical spins can be used to quantify the sc.ple picture of a two-photon cascade and the emission anisotropy of the second radiation. It is a general result ${ }^{8}$ of the angular momentum properties of a photon fjeld that the directional correlation function for a $\gamma_{1}-\gamma_{2}$ cascade can be written as 


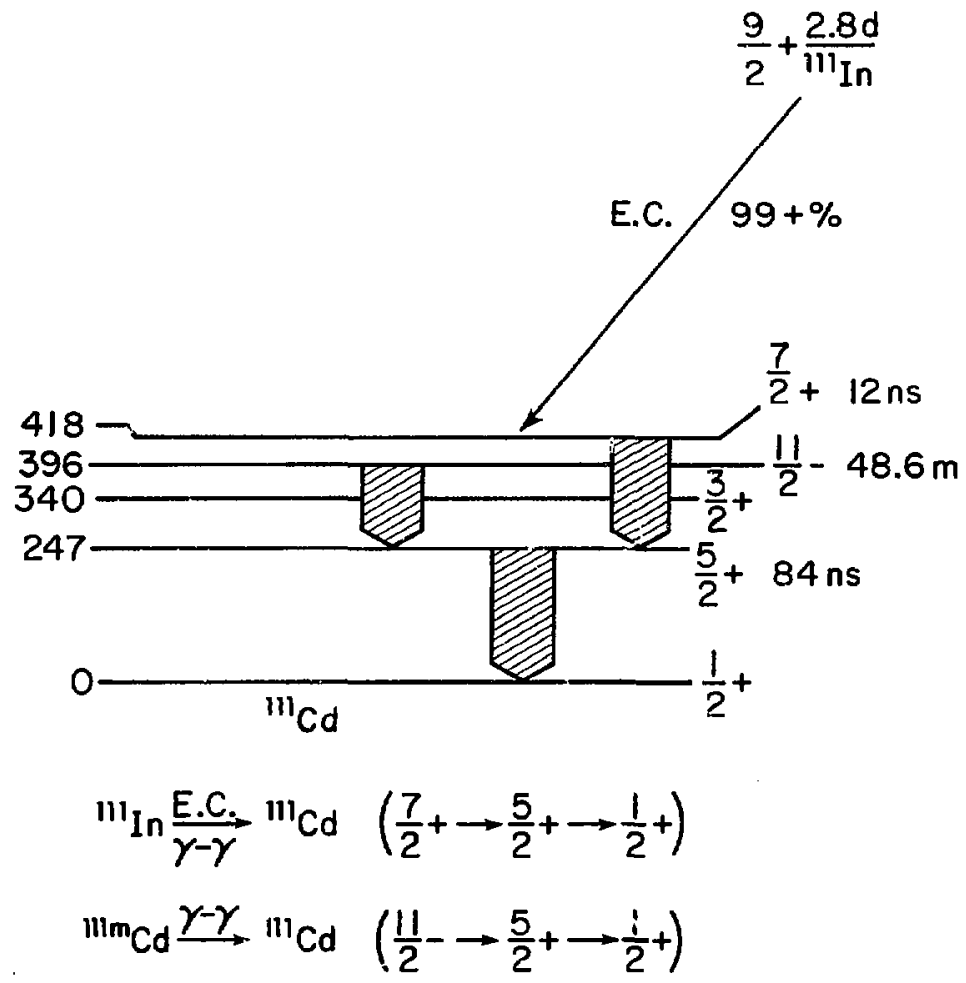

Fig. 11.1 


$$
W(\theta)=\sum_{k} A_{k_{1}} A_{k_{2}} P_{k}(\cos i)
$$

where $k$ takes on only even values for photon-photon correlations and obtains a maximum cutoff value of $2 \mathrm{I}, 2 \ell_{1}$, or $2 \ell_{2}$, whicherer is smallest. Here $I$ is the intermediate level spin and $\ell_{1}$ and $\ell_{2}$ ar: the multipolarities of the radiation for the first and secono photons. The $\mathbf{P}_{k}\{\cos \theta)$ are the Legendre polynonials, and for pure multipole radiation the $A_{k}$ 's are functions of the multipole order and the initial and final spins of the states connected by the transition. For purposes of the ${ }^{111} \mathrm{~m} \mathrm{Cd}$ and ${ }^{111}$ In cascades (Fig. 1), $\mathrm{k}$ can be effectively truncated at 2 and the normalized unperturbed correlation function written as:

$$
W(\theta)=1+A_{22} P_{2}(\cos \theta) \text {, where } A_{22} \equiv A_{k_{1}} A_{k_{2}} \text { for } k=2 \text {. }
$$

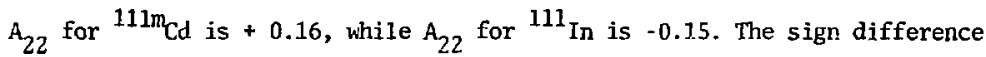
is due to the nuclear spin sequences and photon nultipolarities involved in the two different decay schemes.

B. The Perturbed Directicnal Correlation Function for Static Interactions

A few words are in order concerning the physical meaning of a "perturbed" directional angular correlation. Directicnal correlations in the absence of static magnetic fields or electric field gradients will not depend on the time e?apsed by the nucleus in the intermediate state. However for a nuclear state which has a non-zero spin or finite quadrupole moment, the presence of static magnetic fields or electric field gradients will cause the nuclear state to precess relative to the quantization axis defined by the perturbing field. In order for the perturbing field to be effective in causing a finite rotation of the nuclear state prior to the emission of a gamma quantum, the state must have a finite lifetime comparable to the time associated wjth the precession 
frequency. This precession of the nuclear state prior to the emission of the second detected photon impresses a quantum beat modulation onto the observed directional correlation pattern due to time dependent repopulation shifts in the emittidig state sublevels.

Under the assumption of "static" perturbing fields the time-modulated perturbed angular correlation becomes ${ }^{6}$

$$
\begin{aligned}
w(\theta, t) & =\sum_{k_{1} N_{1}} \sum_{k_{2} N_{2}} A_{k_{1}} A_{k_{2}}\left[\left(2 k_{1}+1\right)\left(2 k_{2}+1\right)\right]^{-\frac{1}{2}} \\
& \times G_{k_{1} k_{2} N_{2}}^{N_{1} N_{2}}{ }^{Y_{k_{1}}}{ }^{*}\left(\vec{k}_{1}\right) Y_{k_{2}}^{N_{2}}\left(\vec{k}_{2}\right) .
\end{aligned}
$$

For a polycrystalline sample one needs to iniegrate over all possible orientations with the constraint that the angle $\theta$ between the emission directions $\vec{k}_{1}$ and $\vec{k}_{2}$ be held fixed. Truncating the maximum $k$ values at 2 and performing the integration yields

$$
w(6, t)=1+A_{22} G_{22}(t) P_{2}(\cos \theta) .
$$

The perturbation factor $G_{22}(t)$ contains all the time dependence and for static fields has the form ${ }^{6}$

$$
\begin{aligned}
& G_{22}(t)=\sum_{N=-2}^{+2} \sum_{1}, n_{2} m_{1}, m_{2}(-1)^{2 I+m_{1}+m_{2}} \exp \left(-\frac{i}{h}\left[E_{n_{1}}-E_{n_{2}}\right) t\right] \\
& \times\left(\begin{array}{ccc}
I & I & 2 \\
\mid n_{1} & -m_{1} N
\end{array}\right)\left(\begin{array}{lll}
I & I & 2 \\
m_{2}^{\prime}-m_{2} N
\end{array}\right)\left(n_{1}\left|m_{2}\right\rangle^{*}\left(n_{1} \mid m_{1}\right)\left(n_{2}\left|m_{2}\right\rangle\left\langle n_{2}\right| m_{1}\right)^{*} .\right.
\end{aligned}
$$

Here $\left|m_{1}\right\rangle,\left|m_{1}^{\prime}\right\rangle,\left|m_{2}\right\rangle,\left|m_{2}^{\prime}\right\rangle$ are eigenfunctions and $\left|n_{1}\right\rangle,\left|n_{2}\right\rangle$ 
eigenvectors of the Hamiltonian belonging to the eigenvalues $E_{n_{1}}$ and $\mathrm{E}_{\mathrm{n}_{2}}$ respectively. In the presence of both an internal magnetic field $\mathrm{H}_{\text {int }}$ and an slectric field gradient $\mathrm{eq}_{\mathrm{zz}}$, the Hamiltonian describing the interaction with the nuclear spins and quadrupole moment is

$$
\mathcal{C}=-\gamma \mathrm{h} \overrightarrow{\mathrm{H}}_{\text {int }} \cdot \overrightarrow{\mathrm{I}}+\frac{e^{2} q_{z z} Q}{4 I(2 I-1)}\left\{3 I_{z}^{2}-I(I+1)+n_{1}\left(I_{x}^{2}-I_{\gamma}^{2}\right)\right\} .
$$

The components of $\vec{I}$ refer to the principal axes of the field gradient tensor. Our experiments essentially spanned the three possibilities for the Hamiltonian of Eq. 7, i.e. a pure magnetic interaction, a pure electric field gradient, and a combined interaction of the most general sort where the principal axis of the electric field gradient does not coincide with the internal magnetic field direction.

For the purely magnetic interaction involving an intermediate level of spin $5 / 2$ the perturbation factor for a polycrystalline sample is a simple analytic function given by

$$
G_{22}(t)=1 / 5\left(1+2 \cos \omega_{L} t+2 \cos 2 \omega_{L} t\right),
$$

where $\omega_{L}$ is the Lamor precession frequency $g \mu_{N} H_{i n t} / \hbar$.

The Haniltonian for the pure quadrupole case is not diagonal unless the electric field gradient has axial symetry, in which case $n=0$ and the perturbation function for spin $5 / 2$ once again has a simple analytic form

$$
G_{22}(t)=\frac{1}{5} \quad 1+\frac{13}{7} \cos \omega Q t+\frac{10}{7} \cos 2 \omega Q^{t}+\frac{5}{7} \cos \omega 3 Q^{t}
$$

and

$$
\omega_{Q}=3 c^{2} q_{z z} Q / 12 I(2 I-1) .
$$


For electric field gradients lacking axial symetry n will not be zero and $\mathrm{G}_{22}(\mathrm{t})$ must be generated numerically. This is also true of the combined interaction, for which one must diagonajize the Hamiltonian (7) in order to calculate the eigenvalues and eigenvectors needed for the evaluation of $G_{22}(t)$.

C. Applied Magnetic Fields

Certain situations dictate experiments in the parangnetic state, i.e. $H_{\text {int }}=0$. One can still extract data pertinent to the magnetic properties by applying a magnetic field perpendicular to the plane of the dttectors. There is now ore field fixed in space perpendicular to the correlation plane rather than an internal field whose axis is randomly oriented relative to the correlation plane. The perturbed correlation function for a static magnetic field $\mathrm{H}$ perpendicular to the plane of the detectors is given by ${ }^{9}$

$$
W\left(\theta, t, H_{\perp}\right)=\sum_{k \text { even }} b_{k} \cos k\left(\theta-\omega_{L} t\right) .
$$

The same fundamental information is availaile from botn the applied

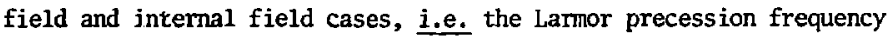
$\omega_{\mathrm{L}}=\mathrm{g} \mu_{\mathrm{n}} \mathrm{H} / \mathrm{h}$, but the Fourier coefficienis of the perturbation factors for the two cases are now different. 
III. APPARATUS

Data for this thesis were collected on two separate $Y-Y$ coincidence spectrometers, one employing four counters and the other eight. We found it advantageous to run both spectrometers simultaneously, usually reserving the eight-counter system for those compounds which would require more statistics in the analysis, e.g. in the case of combined interactions. A given detector is used as both a START and a STOP in the eight-counter system, and it was able to accumulate data at a rate of nearly six times that of the older four-counter systern.

\section{A. The Four-Counter Systen Electronics}

Figure 2 shows the basic schematic for the fou.'-counter systen. The detectors were $1 \times 1 \frac{1}{2}$ " NaI(T1) crystals followed by Amperex 56 DVP photomultipliers and LBL designed preamps. The fast signals were clipped with a shorted delay line and fed into EGGG TD101 differential discriminators. Both START and STOP output pulses from the discriminators strobed an Ortec 437A time-to-amplitude converter (TAC), but the STOP signals were run through a passive delay box prior to entering the TAC in order to provide a time region in which only chance coincidences could be azcumalated. The slow side of the preamps fed LBL designed linear amplifiers and singlem channel analyzers (SCA) and the output signals were fed into a slow coincidence unit. The output of this slow coincidence unit was used to trigser a mixor-delay gate whose input was the TAC output signal, and the gate output which represented the slow-fast coincidence data was fed into a Scipp 1600 channel analyzer with appropriate routing to record two $180^{\circ}$ and twc $90^{\circ}$ spectra. After the spectra had been accumulated on the Scipp, they here 


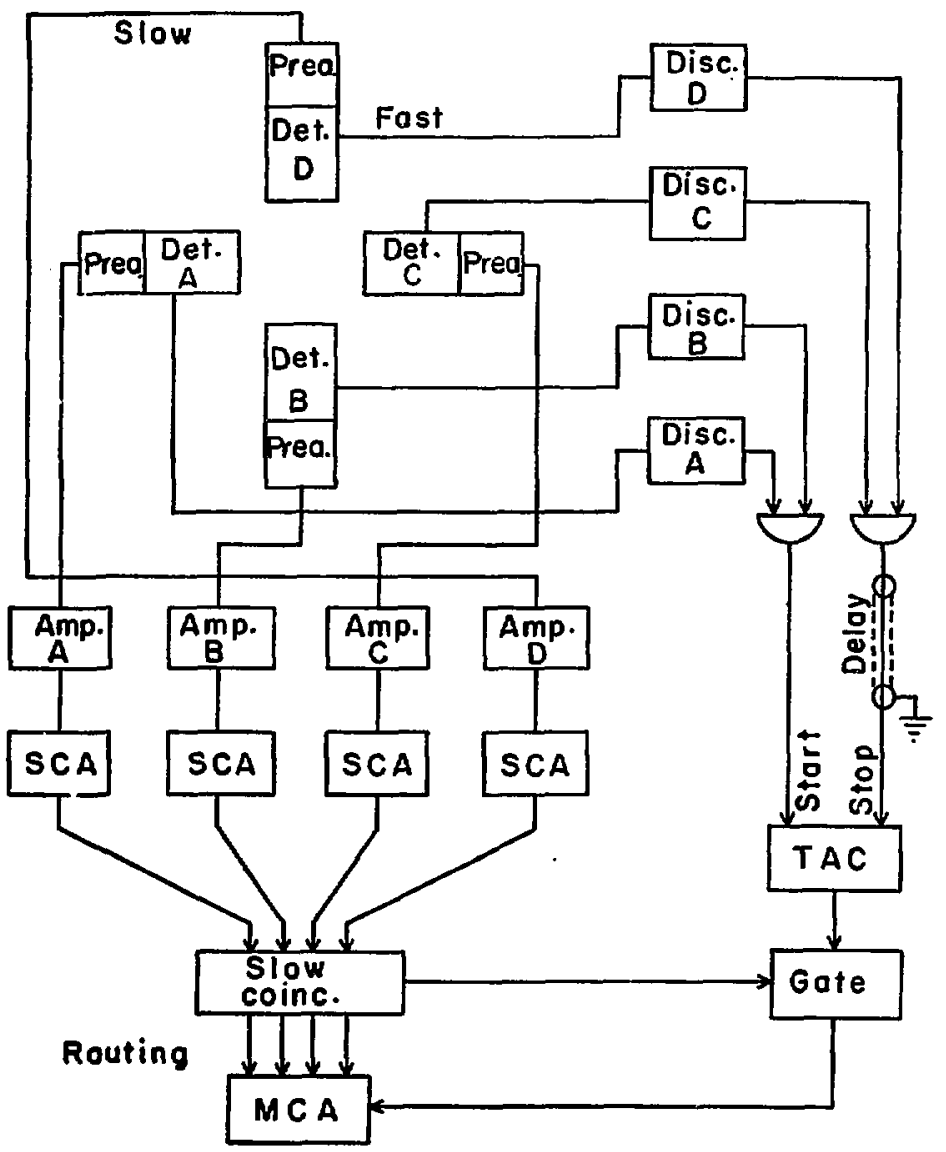

Fig. III-2. 
recorded on a Kennedy Incremental 1500 tape unit for subsequent analysis. The typical time resolution for this system was $1.8 \mathrm{~ns}$ FatM on the ${ }^{22} \mathrm{Na}$ 511-511 cascade with the SCA windows set up for the ${ }^{111 \mathrm{~m}} \mathrm{Cd}$ cascade. An Eldorado 610 Digital Delay Generator and alibrated lengths of delay cable were used to estavish the time calibration.

B. The Eight-Counter System Electronics

The eight-counter system was designed to produce higher counting efficiency and better time resolution than the older system. Figure 3 shows the essential pulse sequencing for one detector. The use of two SCA's plus additional circuitry allowed each detector to be used as both a START and a STOP detector. $1 \times 1 \frac{1}{2}$ ' NaI(Tl) scintillators were used with RCA 8850 photomultiplier tubes which had been selected for minimal gain shifts at high counting rates. The LBL preamps were modified so that the final dynodes were held at a fixed voltage by Zener dioras to further improve the high counting rate capacity of the system. The fast output of the preamps fed constant fraction discriminators of a type described by Maier and Sperr. ${ }^{10}$ The fast pulse shaper produces outputs which feed circuits to suppress multiple coincidences as well as feeding the basic fast timing data into a high speed coincidence circuit simiiar to that described by Gerholm et al. ${ }^{11}$ With this circuitry each detector functioned both as a start and a stop and triple and quadrupole coincidences were suppressed to better than $0.05 \%$ even at high counting rates. The fast coincidence data fed an LBL designed TAC whose output was directed through a delay into the input of a linear gate. The gate trigger was strobed by the slow logic and multiple coincidence suppression circuitry, and the fin; 1 timing information was directed to an 8192 channel Hewlett 


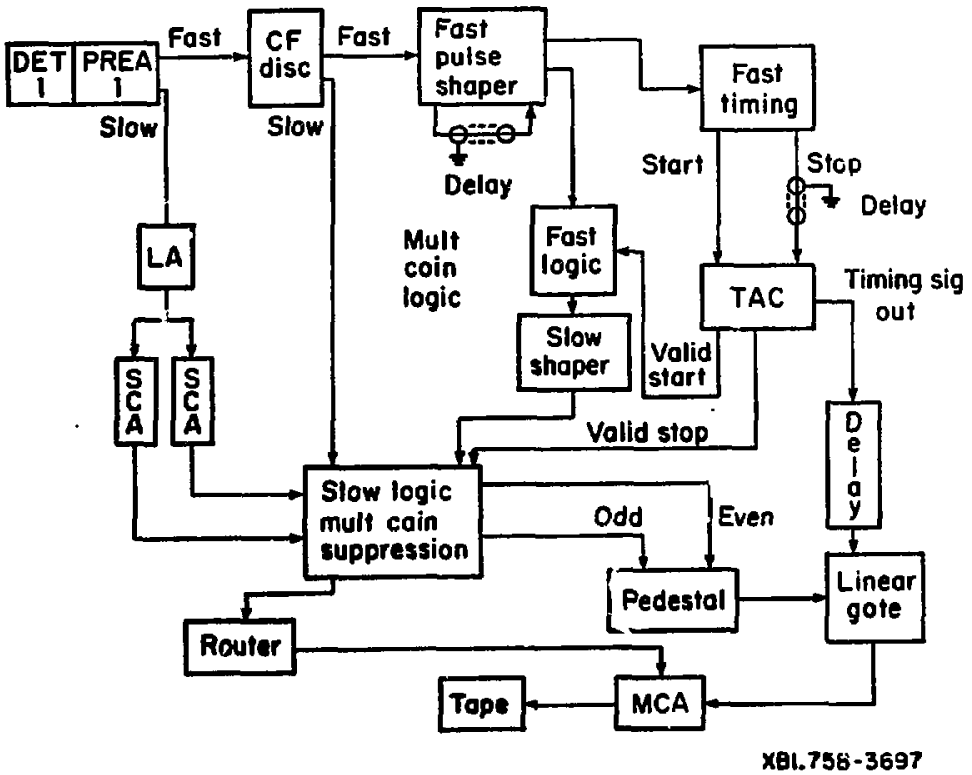

Fig, III-3. 
Packard PHA along with the appropriate :outing signals. A Kennedy 1500 tape unit was coupled to the PHA for purposes of data reduction.

The eight-counter system recorded eight $180^{\circ}$ and eight $90^{\circ}$ spectra and circuit-wise could be split into two (an even and an odd) fourcounter systems. The purpose of the pedestal was to add in the ever side a region which reflected only chance coincidences. This could not be incorporated into a simple delay box as with the odd side because of the way the TAC was designed. The characteristic time resolutior: of this system was 1.05-1.15 ns FWM on ${ }^{22} \mathrm{Na} 511-511$ cascade with the SCA's set up for the ${ }^{111 \mathrm{n}}$ Cd cascade. The Eldorado 610 Digital Delay Generator was used to calibrate the TAC.

C. The High-Pressure Cell, Temperature Regulator, and Miscellaneous Equipment

In order to measure the pessure dependence of t.de transferred hy perfine field, we built a simple clamping-type pressure cell which is shown schematically in Fig. 4. "Windows" were machined out of the cell in the vicinity of the sample in order to obtain the highest possible coincidence rate. We chose to make the cell body out of A286, a steel which does not fracture at liquid helium temperatures. The clamping nut and backup plugs are also of $\mathbf{1 2 8 6}$, while the pistons were ground from tungsten carbide. The cell worked reliably to 22 kbar but at pressures around 30 kbar the cell body and inner borr tended to stretch. After something like thirty nus the inner bore had stretched non-uniformly from 187.5 mils to 196 mils. Ihe pressure locked into the cell after tightening the clamping nut was cill ibraterl by measuring the shift in the quadrupole coupling constallt for 111 ln in tid netal. 

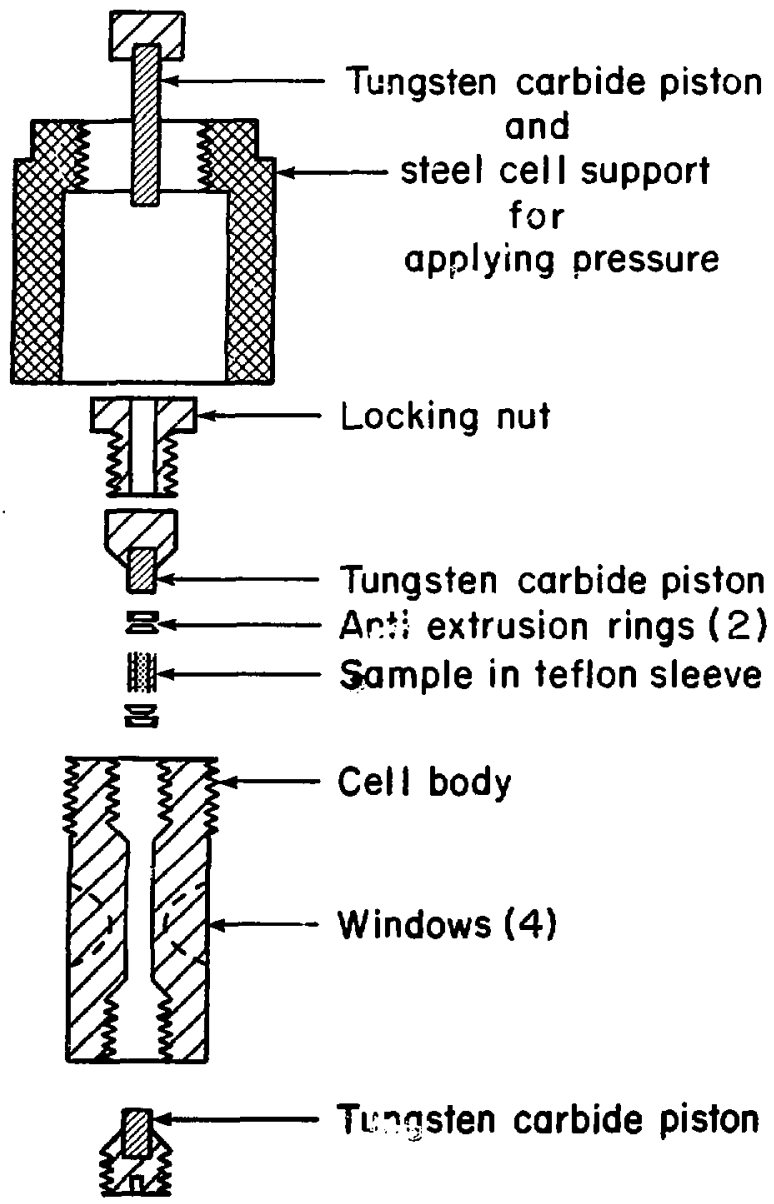

lig. 11-4.

XBL 758-3696 
For experimerits in which the temperature of the sample needed to be regulated, we modified a standard metal dewar with windows for low znergy $\gamma$-ray counting by necking down the tailstock to $\frac{1}{2} "$ id. Into this reduced tailstock we inse:ted our sample holder and temperature regulator of the type first described by Welber and Tynan. ${ }^{12}$ Figure 5 shows our adaptation, which cousists of nothing more than a thin-walled stainless steel tube connected to four inches of nylon rod and terminated in a copper bcbbin to which the aluminum sample housing wes connected. The nylon rod was pressed through a four inch cylinder of styrofoan which fit snugly into the tailsto:k and prevented liquid helium from passing to the sample holder. The copper bobbin had a heater and fu-Fe thermocouple attached to it for purposes of temperature control and measurement. We calibrated the thermocouple against a gemanium thermometer purchased from Cryo. Cal, Inc The calibration of the germanitun themometer was traceable from NBS standards from 1.5 to 100 degrees Kelvin.

Figure 6 shows a simple schematic for the circuit employed in the actual temperature regulation. During a measurement the thermocouple voltage was fixed on a Leeds Northrup $\mathrm{K}-3$ potentiometer and the out of balance signal displayed on a galvanometer. Fluctuations of the out of balance signal were detected by a light sensing djode and fed back into the henter on the copper bobbin. Regulation was stable over several hairs to $\pm 0.2^{\circ} \mathrm{K}$ for temperatures greater than $15^{\circ} \mathrm{K}$, but mlow this temperature the response of the feedb. ck was insufficient to regulate properly.

Two different magnet configurntions were used in the work. Fur the paramignetic shift experiments a C-frame iron core electronggnet with a 


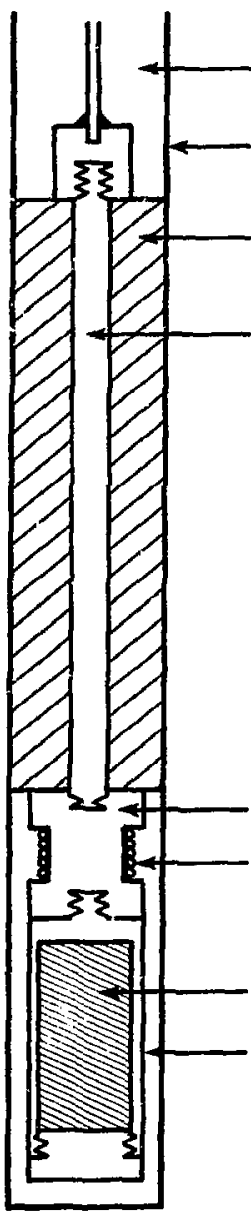

He bath

Dewar wall

Styrofoam

Nylon rod

Cu bobbin

Heater and thermocouple

Sample

Al sample holder

Fig, $111-5$. 


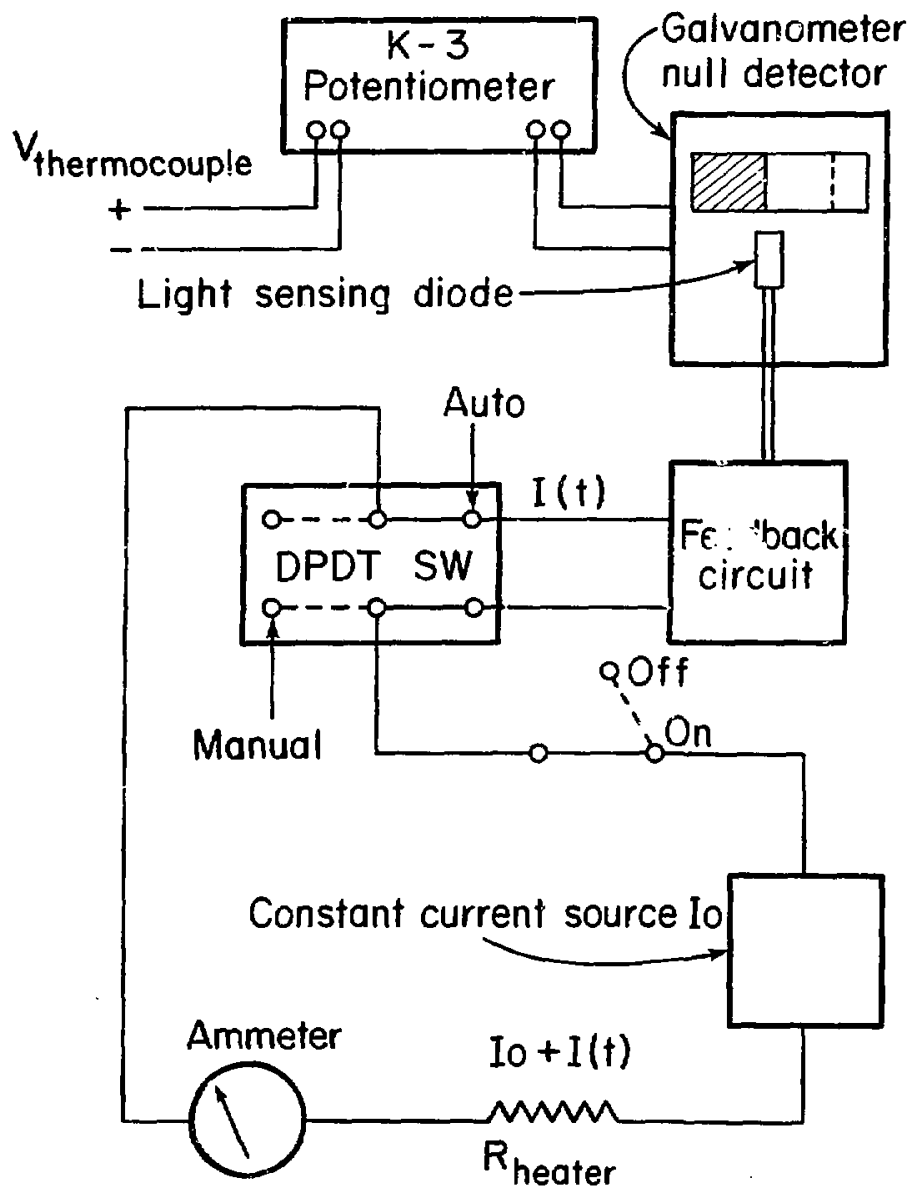

Fig. III-6. 
maximm field of 38 kOe with a one inch gap sufficed. The magnet was powered by a Spectromagnetic Industries Model TC 200-300 D.C. power supply with a stability of $1 \%$ over several days. The spin-flop experiment required counting along the magnetic field axis, and for this purpose we bored out the pole pieces of a small Varian NMR magnet and inserted the counters parallel to the applied field.

The fields of the magnets were necured using a rotating coil gaussmeter which had been calibrated against an NMR probe in a high homogeneity electromagnet. The field errors were principallv due to current supply drift and nonreproducible sampling of the voliune between the pole pieces. 


\section{DATA ANALYSIS}

Since the eight-counter circuitry is consistent with a description as two high-speed four counter systems, it shall suffice to describe how the data are analyzed for a set of four counters placed on a square array and labeled cyclically A,B,C,D. If a given detector can function as only a STAR' or a STOP, then there would be two $180^{\circ}$ coincidences $(A C, B D)$ and four $90^{\circ}$ coincidences $(A B, B C, C D, D A)$. The eight-counter electronics allow a counter to function as both a START and a STOP detector, thereby doubling the number of allowed combinatjons. In practice all of the $180^{\circ}$ spectra are used but only half of the $90^{\circ}$ combinations are selected for analysis. The coincidence counting rate between two detectors, e.g. $A$ and $C$, is related to the polycrystalline correlation function $\left(k_{\max }=2\right)$ found in kq. 5 by the following expression

$$
N_{A C}(t)=N_{0} e^{-t / T} E_{A C}\left\{1+Q_{A C} A_{22} G_{22}(t) P_{2}\left(\cos \theta_{A C}\right)\right\}+C_{A C}
$$

$\mathrm{N}_{0}$ reflects the source strength when the experiment begins, and $E_{A C}$ is an "effective" detector efficiency for the detection of the $\gamma_{1}-\gamma_{2}$ pair by NaI (T1) crystals of fixed thickness. The factor $e^{-t / \tau} N$ describes the fact that even without a perturbation a statistical ensemble of muclei, each with a mean life $\tau_{N}$, will decay exponentially, $Q_{A C}$ accounts for the finite solid angle which the detectors subtend, and the constant $\mathrm{C}_{\mathrm{AC}}$ reflects the background of chance coincidences.

\section{A. Extraction of the Factor $\mathrm{A}_{22} \mathrm{C}_{22}(\mathrm{t})$}

It was realized ${ }^{13}$ some $t$ ime ago that the coincidence counts of the onbinitions $\mathrm{N}_{\mathrm{AC}}\left(180^{\circ}\right), \mathrm{N}_{\mathrm{BD}}\left(180^{\circ}\right), \mathrm{N}_{\mathrm{AD}}\left(90^{\circ}\right)$, and $\mathrm{N}_{\mathrm{BC}}\left(90^{\circ}\right)$ could be arranged 
in such a way that the source strength $N_{0}$, the nuclear lifetime $\tau_{N}$, and to a good approximation the coincidence efficiencies $E_{A C}$, etc. could be made to cancel. This is not true if one has only two or three counters, where unequal coincidence efficiencies and solid angle factors necessitate large corrections. This is accomplished by using detectors $A$ and $B$ as STARTS, and $C$ and $D$ as STOPS, and by forming the ratio

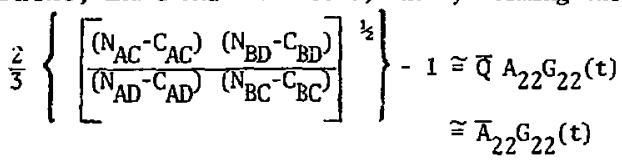

Here $\bar{Q}$ is an average solid angle factor which can be incorporated into the theoretical $A_{22}$ value derived from the spin levels and maltiplicities to yield an "effective" amplitude $\bar{A}_{22}$.

B. Least Squares Fitting of $\bar{A}_{22} G_{22}(t)$

Having extracted the factor $\bar{A}_{22} G_{22}(t)$, it is necessary to fit the perturbation in order to obtain the Lamor and quadrupole radial frequencies ${ }^{L} \mathrm{~L}$ and $\omega_{\mathrm{Q}}$. For a pure magnetic interaction the spectra were fit to

$$
\bar{A}_{22} G_{22}(t)=\frac{\bar{A}_{22}}{5}\left\{1+2 \cos \omega_{L}\left(t-t_{0}\right)+2 \cos 2 \omega_{L}\left(t-t_{0}\right)\right\}+\text { back. }
$$

and for a pure quadrupole interaction with axial symetry $(n=0)$

$$
\begin{aligned}
A_{22} G_{22}(t) & =\frac{\bar{A}_{22}}{5}\left\{1+\frac{13}{7} \cos \omega_{Q}\left(t-t_{0}\right)+\frac{10}{7} \cos 2 \omega_{Q}\left(t-t_{0}\right)\right. \\
& \left.+\frac{5}{7} \cos 3 \omega_{Q}\left(t-t_{0}\right)\right\}+ \text { Back. }
\end{aligned}
$$

The fit parameters were $\vec{A}_{22}, \omega_{L}, \omega_{Q}, t_{0}$, a zero channel for starting the 
the digital data, and Back, a backoround generally due to self-absorption in the samples. Occasionally we experienced a distribution of fields, and this was accounted for by introducing a factor multiplying the $\cos n \omega\left(t-t_{0}\right)$ coefficients by $e^{-\left|n \omega \sigma\left(t-t_{0}\right)\right|}$ or $e^{-\frac{1}{5}\left(\sigma n\left(t-t_{0}\right)\right)^{2}}$ for Lorentzian or Gaussian distributions. In this case $\sigma$, the full width at half maximum, became an additional parameter.

For a quadrupole interaction with a non-zero asymetry parameter, the ratio of the frequencies in Eq. 15 are no longer 1:2:3. A numerical solution of the secular equation then yielded the eigenvectors for the Hamiltonian. In order to guess a reasonable value for the asymmetry parameter $\eta$, we would Fourjer transform the data in order to extract the ratios of the frequiencies $\omega_{1}: \omega_{2}: \omega_{3}$. These ratios could then be compared with the derived ${ }^{14}$ ratios for various values of $n$ for a spin $5 / 2$. It was generally necessary to have an initial guess for the parameter values which was fairly close to the true values in order for the fitting program to converge. This was especially true when performing five and six parameter fits.

For the general case of combined interactions, the Fourier transform of the autocorrelation function of the digital data was taken and compared with a set of computer generated theoretical curves for various values of the magnetic field and electric field gradient parameters. It was necessary to run the spectrum above the magnetic transition in order to obtain a value for $\omega_{Q}$ and $n$ which would be close to the ones obtained in the liquid helium spectra. It was also necessary to guess the angle $\theta$ between the principal axis of the electric field gradient and the internal magnet ic ficld irom symet $y$ considerat ions. With those constraints one could 
run theoretical Fourier transform spectra as a function of internal magnetic field only and compare thent to the experinental transforms. This matchup would then constitute a set of initial guesses for the parameters in the least squares fitting routine. 


\section{ACIADLLLDCEAENTS}

At this poiat is is natural to stop and reflect on the years spent in ary thes is effort. My parting impressions are concemed less with the projects undertaken thin with the my people tho helped to shore and shape the experiences of the last several years. It is witi particular gratitude that I wisk to thank bavid $A$. Shirley and therpert Riuncherg for their guidance, patience and encouragement in directing my rescarch. of no less inportance are those close friends and acquaintances whose contributions cannot be weighed in terass of ideas shared or technical support rendered. I take a special pleasure in thanking Baylor and $L$ inda Triplett, Larn Bermstein, Wini leppler, Corla Bolz, Betty James and Gus Apai for helping to make the years pass in a pleasant and friendly fashion. I would also like to single out those zechnical support groups whose chergy and competence place then above the already excellent levels available at LBI.. Necolades are due the entire staff of the Chenistry Department Machine Shop, the Ikalth Chemistry monitors who regularly participated in my target preparations, and Ruth Mary Lariner and larry Harrington of the 88 inch LBL cyclotron. 


\section{REFIRRACES}

1. Linus Pauling, J. Am. Chenl. Soc. 53, 1367 (1931); Phys. Rev. 37, 1185 (1931); J. Am. Chem. Soc. S4, 988 (1932).

2. J. H. Van Vleck, Phys, Rev, 41, 208 (1932).

3. H. A. Kraners Physica 1, 182 (1934).

^. P. W. Anderson, Solid State Phys. 14, 99 (1963); Informal Proc. Buhl Intem. Conf. Mater. Pittsburgh, p. 17 (1963).

5. H. Frauenfelder and R. M. Steffen, Angular Correlations in Alpha-, Beta-, and Gama-Ray Spectroscopy, K. Siegbahn, editor (North Holland Pub. Co., Amsterdam, 1965), p. 997 ff.

6. E. Karlsson, E. Matthias, and K. Siegbahn, editors, Perturbed Angular Correlations (North Holland Pub. Co., Ansterdan, 1964).

7. B. M. Brisk and G. R. Satchler, Angular Momentum (Clarendon Press, Oxford, 1968).

8. S. G. Cohen, Chap. 12, Hyperfine Interactions, A. J. Freeman and R. ᄀ. Frankel, editors, (Acadenic Press, New York, London, 1967).

9. A. J. Ferguson, Angular Correlation Methods in Gamma-Ray Spectroscopy (North Holland Pub. Co., Ansterdan, 1965).

10. M. R. Maier and P. Sperr, Nucl. Instr. Methods 87, 13 (1970).

11. T. R. Gerholn, Z. H. Cho, L. Eriksson, L. Gidefeldt, and B. G. Pettersson, Nucl. Instr. Methods 100, 33 (1972).

12. B. Welber and E. E. Tynan, Rev. Sci. Instrum. 38(1), 137 (1967).

13. B. Erik Karlsson, Arkiv. Fysik 22, 1 (1962).

14. E. Gerdau, J. Wolf, H. Winkler, and J. Braunsfurth, Proc. Roy. Soc. A. $\underline{311}, 197$ (1969). 
FIGURE CAPTIONS

Fig. 1. Two photon decay cascades for ${ }^{111} \mathrm{~m}$ d and ${ }^{111}$ In used ir this work.

Fig. 2. Electronic schematic of the four-counter system showing the components and their sequencing.

Fig. 3. Block diagram for a single NaI(T1) counter in the elght-counter system showing the electronic sequencing of the fast and slow signals.

Fig. 4. High pressure clamping type pressure cell. The body is constructed of A286 steel and four "windows" have been machined in tine vicinity of the sample to reduce gama-ray attenuation.

Fig.. . The temperature regulator employed in the sublattice magnetization experiments shown enclosed in the tailstock of a liquid helium dewar.

Fig. 6. Block diagram of the temperature regulator electronics. The light sensing diode was linked to a feedback circuit which pulsed the sample heater and regulated the temperature over several hours to $\pm 0.2^{\circ} \mathrm{K}$. 


\section{SYSTTYATICS OF IMPERFINE FIILLS OHSLRVED BY PAC} IN Cl-DOPIJ) IRANSITION HETAL ANTIFERROAMONETS

\section{A. INTRODUCTION}

The properties of the simple 3d transition metal salts with $d^{5}$ through $d^{10}$ configurations are generally consistent with localized elestron behavior, ${ }^{1}$ for which LCAD-ND calculations can describe the nature of the chemical bonding with reasonible success. Within the framework of the Mo model, we have examined the systematic variation of the hyperfine fields transferred through the ligands into a diamagnetic dopant $\left({ }^{111} \mathrm{~m} c d\right)$ for the transition metal using $\gamma$-ray perturbed angular correlation (PAC). The variations of the MD spin density paraneters with changes in both the ligand and the transition metal are correlated with the transferred hyperfine fields.

The PAC data are complenentary to those previuusly derived from MR, ESR, ENDOR, neutron diffraction, and Mössbauer techniques, but there are differences among the methods which deserve some coment. Especially relevant to PAC is a symmetry argument ${ }^{2}$ based on the assumption that the hyperfine field at the Cd impurity is prinarily derived from overlap and covalency effects of the cd ns shells. The symetry of the shells leads to PAC being $\sigma, s$ bond specific. For $d^{5}$ ions the importance of this difference is highlighted by the measurements of $M R_{\text {, }}$ ENDOR, and ESR, which measure $f_{\sigma}-f_{\pi}$, and for neutron diffraction which measures $2 f_{\pi}+f_{\sigma}+f_{S}$. This has hampere? the understanding of the Non compounds, because $f_{\sigma}$ and $f_{\pi}$ are generally of the same order of magnitude. Mösbauer spectroscopy and

This section of work was done in conjunction with H.H. Rinneherg and D. A. Shirley 
PAC are potentially competitive, but there has been little overlap due to the linitations on available Nossbauer nuclei which can be uszd to replace the transition metal substitutionally.

There is an additional problen concerning the comparison of data collected in magnetically dilute (ENOR, ESR) and magnetically concentrated (MR, neutron diffraction, PAC, Mössbauer) systens. Our data are consistent with the vicw that it is val id to compare derived spin density parameters between dilute and concentrated systens within the experimental errors inherent in the data analysis.

Experimental details are given in Section B, while Section C outlines the $\mathrm{NO}$ model and inherent $\mathrm{limitations}$ in its application to the properties of these salts. Section $D$ contains our results and their interpretation relative to the Mo model.

\section{B. EXPERIMENTAI}

\section{Detector System}

Data for this study were accumulated on both four and eight counter $\gamma-\gamma$ coincidence spectrometers which have previously been described. 3,4 Both systens employ the familiar fast-slow logic, and only $180^{\circ}$ and $90^{\circ}$ correlations were observed and combined to yield the perturbation factor independent of counter efficiencies and the lifetime of the intermediate nuclear state. 5

Standard $1 \times 11_{2}^{\prime \prime} \mathrm{NaI}(\mathrm{T} 1)$ crystals were used as photon detectors for the ${ }^{111 m} \mathrm{Cd}$ cascade. The time-to-amplitude converters for the two spectrometers were calibrated with cables and an Eldorado 610 digital delay generator. With the single channel analyzer windows set on the ${ }^{111 \mathrm{~m}} \mathrm{Cd}$ energies $(150,247 \mathrm{keV})$, the time resolution using ${ }^{22} \mathrm{Na} 511-511$ radiation 
was 1.8 and 1.1 ns FWM for the four and eight counter spectroneters respectively.

\section{Sample Preparation}

The preparation of some of the compounds $\left(\mathrm{KNiF}_{3}, \mathrm{KCOF}_{3}, \mathrm{RbMhF}_{3} \mathrm{i}^{2}\right.$ $\mathrm{MnO}, \mathrm{COO}, \mathrm{NiO})^{4}$ had been described previously. In general the $111 \mathrm{~m}$.d was obtained by neutron ir.adiation of ${ }^{110} \mathrm{CdO}$, converted to the appropriate aihalide by dissolving in acid and evaporating to drymess, and subsequently thenrally diffused into the flisorides and chlorides listcd in Tables $\mathrm{IA}$ and $\mathrm{BB}$. Since the $\mathrm{A}_{2} \mathrm{MX}$ compounds melt incongruent1y, it was necessary to sinter the activity in at temperatures well below the peritectic. The remaining chlorides and fluorides melt congruently. Single-phase samples were obtained by diffusing the activity into the lattice at temperatures close to the melting points. The hygroscopic rature of many of the reactants required us to perform the preparative operations under dry nitrogen conditions.

The double fluorides and chlorides $\mathrm{AMX}_{3}$ and $\mathrm{A}_{2} \mathrm{MX}_{4}$ were prepared by the corresponding solid-state reactions:

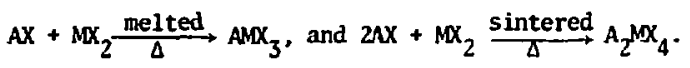

High purity, anhydrous $\mathrm{KF}, \mathrm{RbF}, \mathrm{RbCl}, \mathrm{CoF}_{2}$, and $\mathrm{MnF}_{2}$ were commercially availab]e. T1F was prepared by dissolving $99.999 \mathrm{O} \mathrm{Tl}$ metal in aqueous HF. The product was dried by melting in a platinum cruicible under vacuum. Anhydrous $\mathrm{CuF}_{2}$ and $\mathrm{NiF}_{2}$ were obtained by passing $\mathrm{F}_{2}$ over the respective anhydrous dichlorides at $400^{\circ} \mathrm{C}$. Anhydrous $\mathrm{FeF}_{2}$ was prepared from $\mathrm{FeCl}_{2}$ in a stream of dry $H F$ gas at $650^{\circ} \mathrm{C}$. The transition metal dichlorides had previously been prepared by the dehydration of $\mathrm{AnCl}_{2} \cdot 4 \mathrm{H}_{2} \mathrm{O}, \mathrm{FeCl}_{2} \cdot 4 \mathrm{H}_{2} \mathrm{O}$ 
by a strean of $\mathrm{IHCl}$ gas at $200-300^{\circ} \mathrm{C}$.

a-Nins doped with activity was obtained by coprecipitation under an oxygen-free atmosphere following the procedure given by Brauer. ${ }^{6}$ The precipitate was dried and ther heated to $1000^{\circ} \mathrm{C}$ in a platinum thoe under $\mathrm{H}_{2} \mathrm{~S}$.

\section{Data Aralysis}

For a polycrystalline sample with unique but randomly-oriented axes, the perturbed angular correlation for a $\gamma-\gamma$ cascade is given by ${ }^{7}$

$$
W(\theta, t)=\sum_{k \text { even }}^{\sum} A_{k k} G_{k k}(t) P_{k}(\cos \theta) .
$$

For the ${ }^{111 \mathrm{~m}} \mathrm{Cd}$ cascade it is sufficient to truncate the series at $k=2$ yielding for a normalized correlation

$$
W(\theta, t)=1+A_{22} G_{22}(t) P_{2}(\cos \theta) .
$$

Here $A_{22}$ is the anisotropy of the $150-247 \mathrm{keV}$ cascade, $P_{2}(\cos \theta)$ is the second Legendre polynomial associated with the angle $\theta$ between the direction of the two garma rays, and $G_{22}(t)$ is the polycrystalline perturbation factor. This function is obtained from the magnetic and electric hyperfine interactions of the $\mathrm{Cd}$ nucleus in the intermediate $247-\mathrm{keV}$ state $\left(I=5 / 2, \tau_{N}=121 \mathrm{nsec}\right)$. For a pure ragnetic dipole interaction one obtains an analytical function for the perturbatior. factor ${ }^{7}$

$$
G_{22}(t)=\frac{1}{5}\left\{1+2 \cos \left(\omega_{L} t\right)+2 \cos \left(2 \omega_{L} t\right)\right\}
$$

with $w_{L} \equiv \frac{g_{N}{ }_{n} H_{i n t}}{h}$. i.e., the Larmor precession frequency of the nuclear moment due to the internal magnetic field $H_{\text {int }}$.

In the more general case of a combined magnetic dipole and electric quadrupole interaction, the perturbation factor $G_{22}(t)$ can still be 
calculated as a stm of cosine terms ${ }^{7}$ after obtaining the eigenvalues of the Hamiltonian

$$
H=-g \mu_{n} \vec{H}_{\text {int }} \cdot \vec{I}+\frac{e^{2} q_{z z} Q}{4 I(2 I-1)}\left\{3 I_{z}^{2}-I(I+1)+n\left(I_{x}^{2}-I_{y}^{2}\right)\right\}
$$

The components of refer to the principai axes of the field gradient tensor $\left(e q_{x x}, e q_{y y}, e q_{z z}\right)$ and $n$ is the asymmetry parameter defined by $1=\frac{q_{x x}-q_{y y}}{q_{z z}}$.

In general only for pure magnetic dipole and pure quadrupole interaction with axial symetry $(\eta=0)$ will the frequencies contained in $G_{22}(t)$ be integral multiples of each other and a periodic oscillation of the intensity of the second $\gamma$-radiation be observed. For an asymmetric electric field gradient or a combined magnetic dipole-electric quadrupole interaction, the transition frequencies are no longer overtones of a single frequency due to the shifts of the energy levels of the Hamiltonian, and $G_{22}(t)$ becomes an aperiodic function of time. The spectrun of $\mathrm{KCuF}_{3}$ in the paramagnetic state displays (Fig. 1) a non-axial electric field gradient, while $\mathrm{K}_{2} \mathrm{NiF}_{4}$ (Fig. 2) at $4.2^{\circ} \mathrm{K}$ shows a combined interaction in the antiferromagnetic state, for which the hyperfine field of the inpurity is parallel to the unique axis of the field gravient. In order to anal'ze combined interactions, it is of considerable advantage to calculate ${ }^{8}$ the power spectral density $P(\omega)$ of the perturbition factor rather than $G_{22}(t)$ itself. This is illustrated in Fig. 3 for $\mathrm{K}_{2} \mathrm{NiF}_{4}$ where the solid ine represents a least-squares fit of the power spectral density to that obtained from the experimental data (dotted line). 


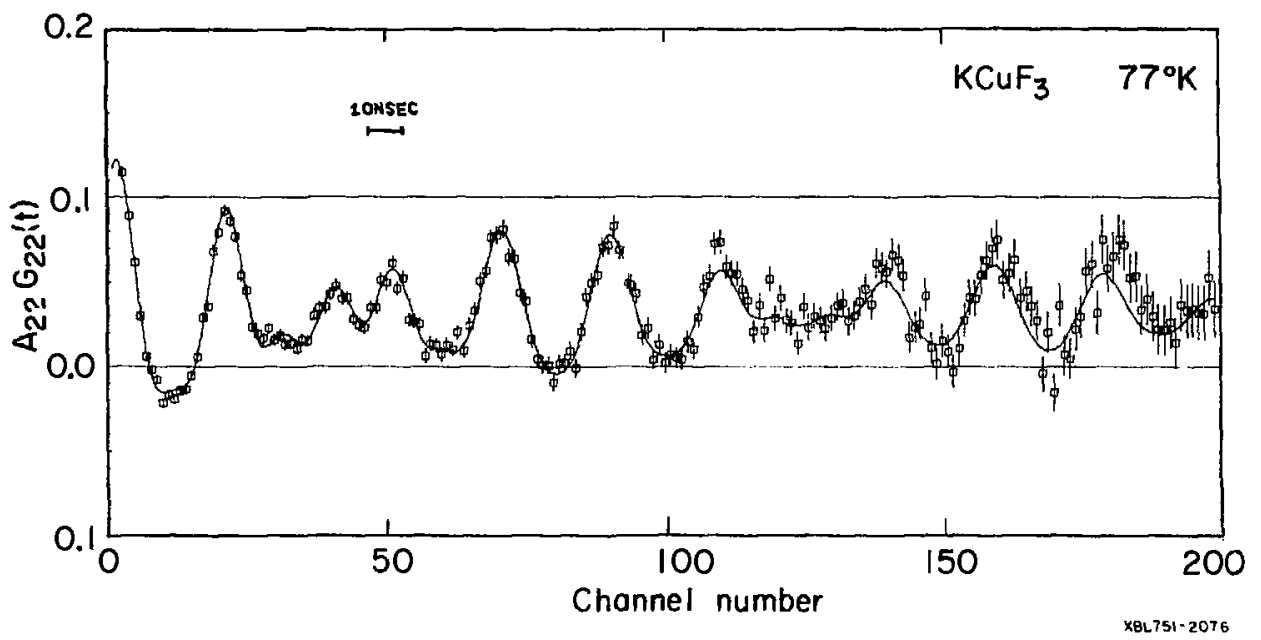

Fig. V-1. 


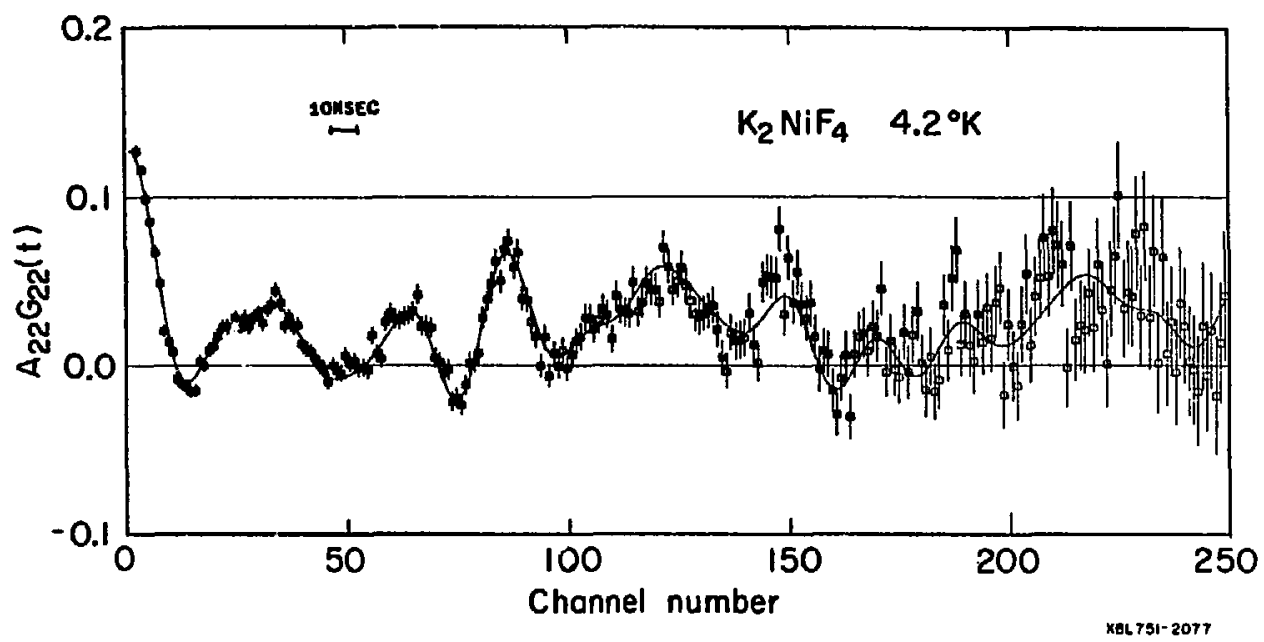

Fig. V-2. 


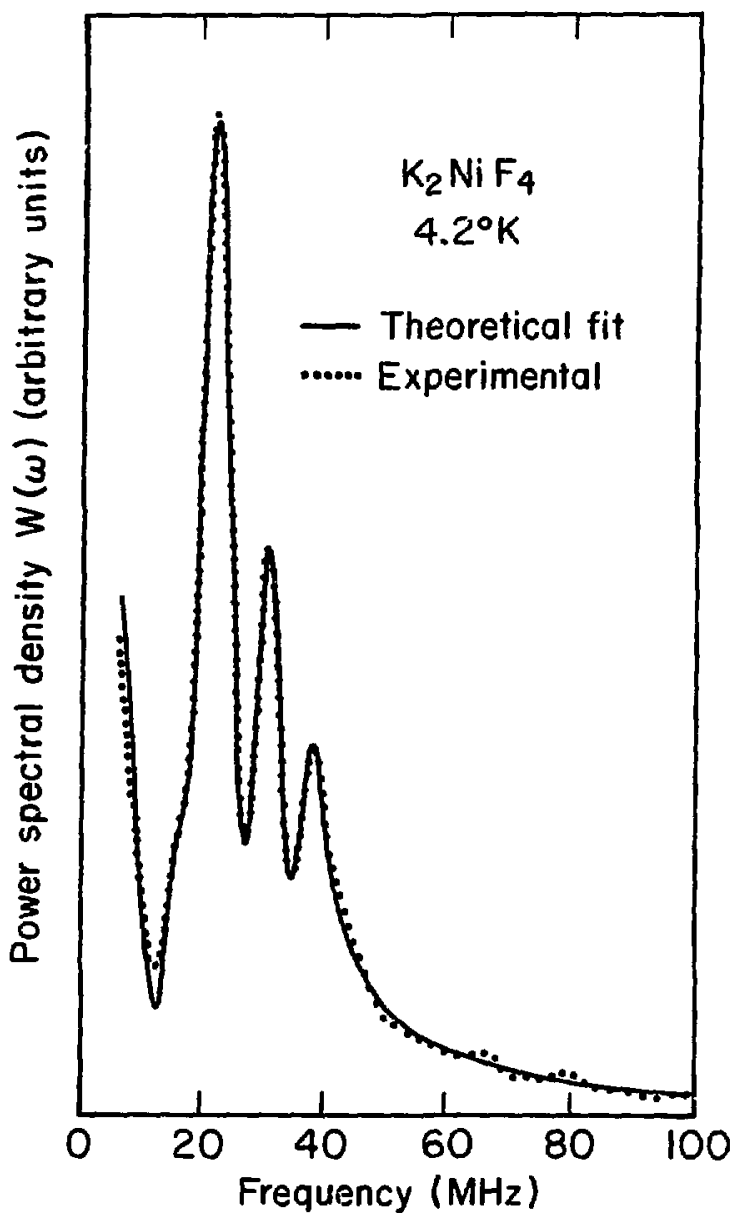

Fig. V-3. XBL75B-3702 
Tables IA,B contain the results of our measurements in insulating ant iferromagnets.

C. LCAO-MD MODEL

Considerable theoretical effort $9,10,11,12,13$ has gone into understanding the electronic energy level diagram of the octahedrally coordinated id transition metal cluster $\left(\mathrm{ML}_{6}\right)^{-\mathrm{q}}$, prinarily with $\mathrm{L}=\mathrm{F}^{-}$or $\mathrm{O}^{2-}$. We will assume the basic correctness of the energy level diagram of Fig. 4 and focus on the properties of the sigma bonds possible between the metal ion and the ligands.

The filled bonding orbitals are primarily of ligand character,

$$
\psi_{\sigma}^{B}=N_{\sigma}^{B}\left(x_{p \sigma}+\gamma_{\sigma} d_{\sigma}+\gamma_{s} x_{s}\right),
$$

where $x_{p o}, x_{s}$ are symmetry-allowed linear combinations of ligand $s$, po orbitals, and $\gamma_{\sigma}, \gamma_{S}$ are covalency mixing parameters which describe the admixing of metal $d_{\sigma}$ orbitals into the ligand $p_{\sigma} s$ orbitals. The ligand orbitals are generally restricted to $2 \mathrm{p}_{\sigma}$ and $2 s$ for $\mathrm{F}^{-}$and $\mathrm{O}^{2-}$, and $3 \mathrm{p}_{\sigma}$ and $3 \mathrm{~s}$ for $\mathrm{Cl}^{-}$and $\mathrm{s}^{2-}$.

The partially-filled antibonding orbitals are mainly metal-like, and contribute to the magnetic properties.

$$
\psi_{\sigma}^{A}=N_{\sigma}^{A}\left\{d_{\sigma}-\left(\lambda_{\sigma} x_{p, J}+\lambda_{s} x_{s}\right)\right\} .
$$

Nomalization and orthogonality of bonding and antibonding orbitals are used to derive relations connecting the parameters. To first order one obtains

$$
\lambda_{\sigma}=\gamma_{\sigma}+s_{\sigma} \quad \text { and } \quad \lambda_{s}=\gamma_{s}+s_{s} .
$$


Tables 1. A,B. Magnetic and quadrupole interactions of ${ }^{111 \mathrm{~m}} \mathrm{Cd}$ in insulat ing host ant ifcrmagnets 1.A. Host matcrials with 3 dimensional magnet ic structure

\begin{tabular}{|c|c|c|c|c|c|c|c|c|}
\hline Host & $\begin{array}{c}T_{N} \\
(K)\end{array}$ & $\frac{H_{\exp }}{(K \theta e \pm 1.5)}$ & $\begin{array}{c}H_{h f} \\
(K \theta e \pm 2.5)\end{array}$ & $\begin{array}{c}{ }^{\mathrm{Q}} \\
(\mathrm{MHz}=0.2)\end{array}$ & $\eta$ & $\begin{array}{l}\text { Crystal } \\
\text { structure }\end{array}$ & $\begin{array}{l}\text { Magnetic } \\
\text { structure }\end{array}$ & Remarks \\
\hline $\mathrm{KMnF}_{3}$ & 95 & 102.6 & & 0 & 0 & \multirow{8}{*}{$\begin{array}{r}\text { Perovskite } \\
\mathrm{KNiF}_{3} \\
\text { type }\end{array}$} & Complex & Spin canting \\
\hline $\mathrm{Rb}^{\prime} \mathrm{HF}_{3}$ & 83 & 113.8 & 117.4 & 0 & 0 & & G & No distortions \\
\hline $\operatorname{TuMnF}_{3}$ & 76 & 118.7 & 122.5 & 0 & 0 & & $\mathbf{G}$ & \\
\hline $\mathrm{KFeF}_{3}$ & 112.5 & 100.1 & 104.2 & $\mathbf{0}$ & 0 & & Complex & Spin canting \\
\hline $\mathrm{KCoF}_{3}$ & 114 & 74.1 & 78.2 & $\mathbf{0}$ & 0 & & \multirow{3}{*}{ G } & \multirow{3}{*}{$\begin{array}{l}\text { Small lattice } \\
\text { distortions }\end{array}$} \\
\hline $\mathrm{RbCOF}_{3}$ & $\sim 101$ & 67.8 & 71.5 & $\mathbf{0}$ & 0 & & & \\
\hline $\mathrm{T} \mathrm{COF}_{3}$ & 94 & 67.9 & 71.6 & 0 & 0 & & & \\
\hline $\mathrm{KNiF}_{3}$ & 253 & 104.8 & 113.7 & $\mathbf{0}$ & $\mathbf{0}$ & & G & No distortion \\
\hline $\mathrm{KCl}_{3}$ & 38,20 & $<4$ & & 19.3 & 0.74 & $\mathrm{KCuF}_{3}$ & A & 2 Modifications \\
\hline Mno & -118 & 202 & 208 & 0 & 0 & \multirow{3}{*}{$\mathrm{NaCl}$} & Mno & $\vec{s} \|\langle\|\|\rangle$ \\
\hline $\mathrm{CoO}$ & $\sim 293$ & 177 & 187 & 0 & $\mathbf{0}$ & & Complex & \\
\hline $\mathrm{NiO}$ & $\sim 520$ & 196 & 212 & 0 & 0 & & $M \neq 10$ & $\vec{s} \|\langle\|\rangle$ \\
\hline $\mathrm{FeCl}_{2}$ & 23.5 & 4.8 & & 5.8 & 0 & \multirow{2}{*}{$\mathrm{CdCl}_{2}$} & $\stackrel{\mathrm{S}}{\mathrm{HC}}$ & \\
\hline $\mathrm{CoCl}_{2}$ & 25 & $\sim 0$ & & 5.8 & $\mathbf{0}$ & & șlC & \\
\hline$\alpha-\operatorname{MrS}$ & 156 & 244.4 & 258 & 0 & 0 & $\mathrm{NaCl}$ & Mno & \\
\hline
\end{tabular}


Table I.B. Host materials with 2 dimensional magnetic structure

\begin{tabular}{|c|c|c|c|c|c|c|c|c|}
\hline Host & $\begin{array}{l}\mathrm{T}_{\mathrm{N}} \\
{ }^{\mathrm{N}} \mathrm{K} \\
\end{array}$ & $\begin{array}{c}11 \exp \\
\left(K \theta \mathrm{e}^{ \pm} 1.5\right)\end{array}$ & $\begin{array}{c}l_{\mathrm{nf}} \\
\left(\mathrm{K} \theta \mathrm{e}^{ \pm} 2.5\right)\end{array}$ & $\begin{array}{c}{ }_{\mathrm{Q}} \\
\left(M \mathrm{t}^{ \pm} 0.2\right)\end{array}$ & $n$ & $\begin{array}{l}\text { Crystal } \\
\text { structure }\end{array}$ & $\begin{array}{l}\text { Magnetic } \\
\text { structure }\end{array}$ & Remarks \\
\hline$K_{2} \operatorname{Mnn} F_{4}$ & -45 & 71.6 & 79.4 & 3.4 & 0 & \multirow{7}{*}{$\mathrm{K}_{2} \mathrm{NiF}_{4}$} & \multirow{7}{*}{ sıc } & \multirow{4}{*}{$\begin{array}{l}\text { Heisenberg } \\
\text { ant iferromagnets } \\
\text { Ising systems- no } \\
\text { zero pt spin deviation } \\
\text { dipole correcticn only }\end{array}$} \\
\hline $\mathrm{Rb}_{2} \mathrm{NnF}_{4}$ & 38.5 & 67.9 & 75.4 & 4.7 & $\mathbf{0}$ & & & \\
\hline $\mathrm{K}_{2} \mathrm{CoF}_{4}$ & 107 & 51.5 & 52.7 & 5.5 & 0 & & & \\
\hline $\mathrm{Rb}_{2} \mathrm{CoF}_{4}$ & 101 & 48.9 & 50.0 & 8.2 & $\mathbf{0}$ & & & \\
\hline $\mathrm{K}_{2} \mathrm{NiF}_{4}$ & -97 & 54.9 & 69.3 & 3.7 & 0 & & & \multirow{2}{*}{ Heisenberg } \\
\hline $\mathrm{Rb}_{2} \mathrm{NiF}_{4}$ & 95 & 51.3 & 64.7 & 7.4 & 0 & & & \\
\hline $\mathrm{Rb}_{2} \mathrm{SnCl}_{4}$ & 57 & 97.4 & 106.7 & 8.1 & 0 & & & Ant iferromagnets \\
\hline
\end{tabular}




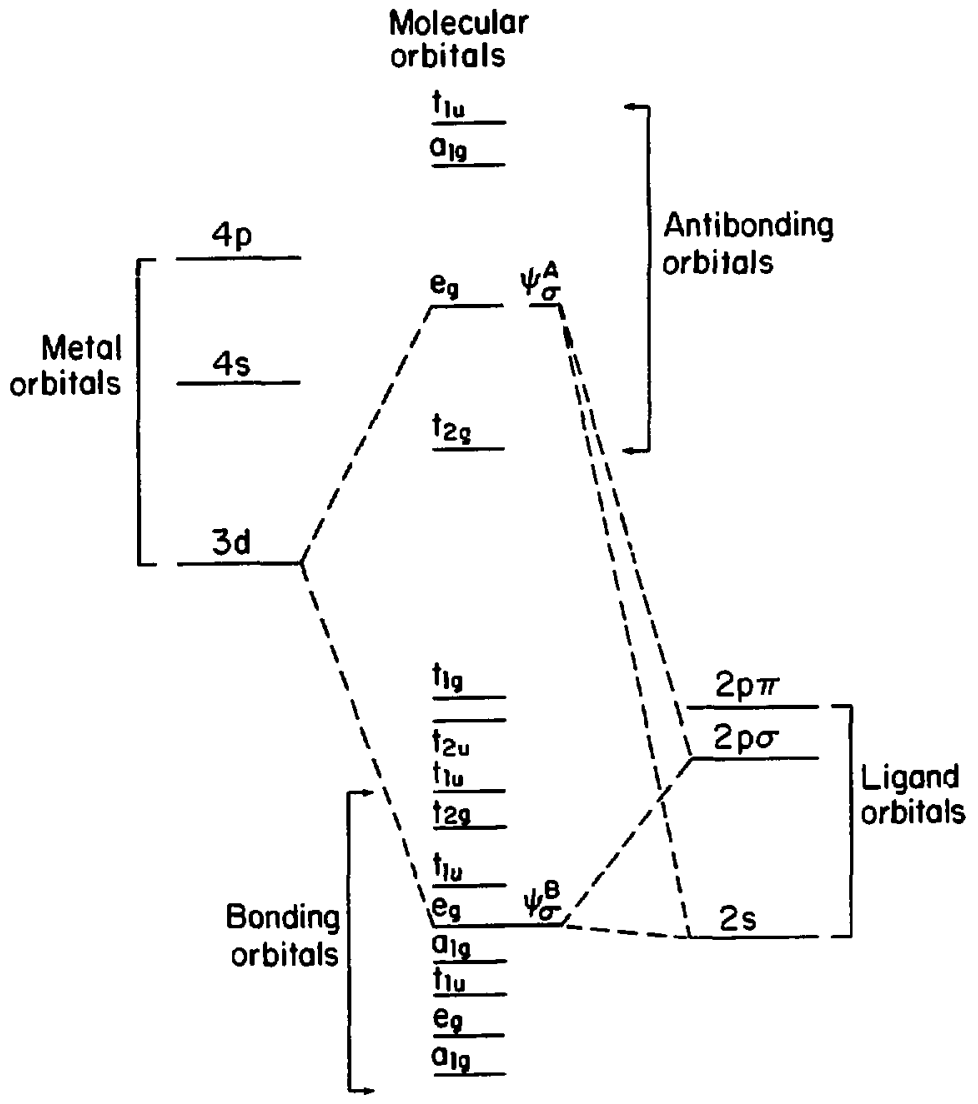

Fig. V-4. 
The overlap integrals $S_{\sigma}$ and $S_{S}$ come from the normalization procedure. They are defined by $s_{\sigma}=\left\langle d_{\sigma} \mid x_{p \sigma}\right\rangle$ and $s_{s}=\left\langle d_{s} \mid x_{s}\right\rangle$.

The unpaired $\sigma$ spin density at the liegand has been the object of numerous experimental endeavors because its precise detemination is fundamental to theoreticai efforts to understand the bonding in these compounds. Using the framework of the LCAO-MO model, Sawatzky and coworkers ${ }^{14,15,16}$ have pointed $\because$, he necessity of considering the entire extended octahedral complex (see Fig. 5) in order to obtain the correct normalization constants for the cluster. This is in contrast to treating the problem as six separate bonds and multiplying the final calculated hyperfine fields by six.

Following Sawatzky's extended cluster calculation and adapting his results to $180^{\circ}$ metal-ligand-Cd exchange paths, we obtain for the supertransferred hyperfine field at the cd nucleus

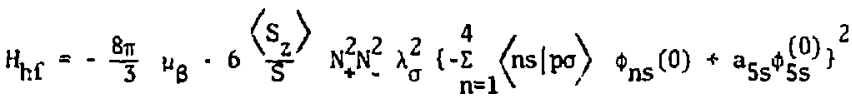

where $\lambda_{\sigma} \equiv \gamma_{\sigma}+\left\langle p o \mid d_{2}\right\rangle$ and

$$
\begin{aligned}
& \left(N_{+}\right)^{-2} \cong\left(N_{-}\right)^{-2}+\lambda_{\sigma}^{2} \\
& \left(N_{-}\right)^{-2}=1-6 \sum_{n=1}^{4}\langle n s \mid p \sigma\rangle^{2}+6 a_{5 s}^{2}+12 a_{5 s}\langle 5 s \mid p \sigma\rangle-\left\langle p \sigma \mid \alpha_{2}\right\rangle^{2} .
\end{aligned}
$$

In this formulation we have omitted the contribution of the ligand $s$ orbitals to the $\sigma$ bonding and have neglected ligand three-center overlaps. The parameter $a_{5 s}$ allows for a charge transfer into the unoccupied $\mathrm{Cd}^{2+} 5 \mathrm{~s}$ orbital yielding a total $5 \mathrm{~s}$ occupation number of 


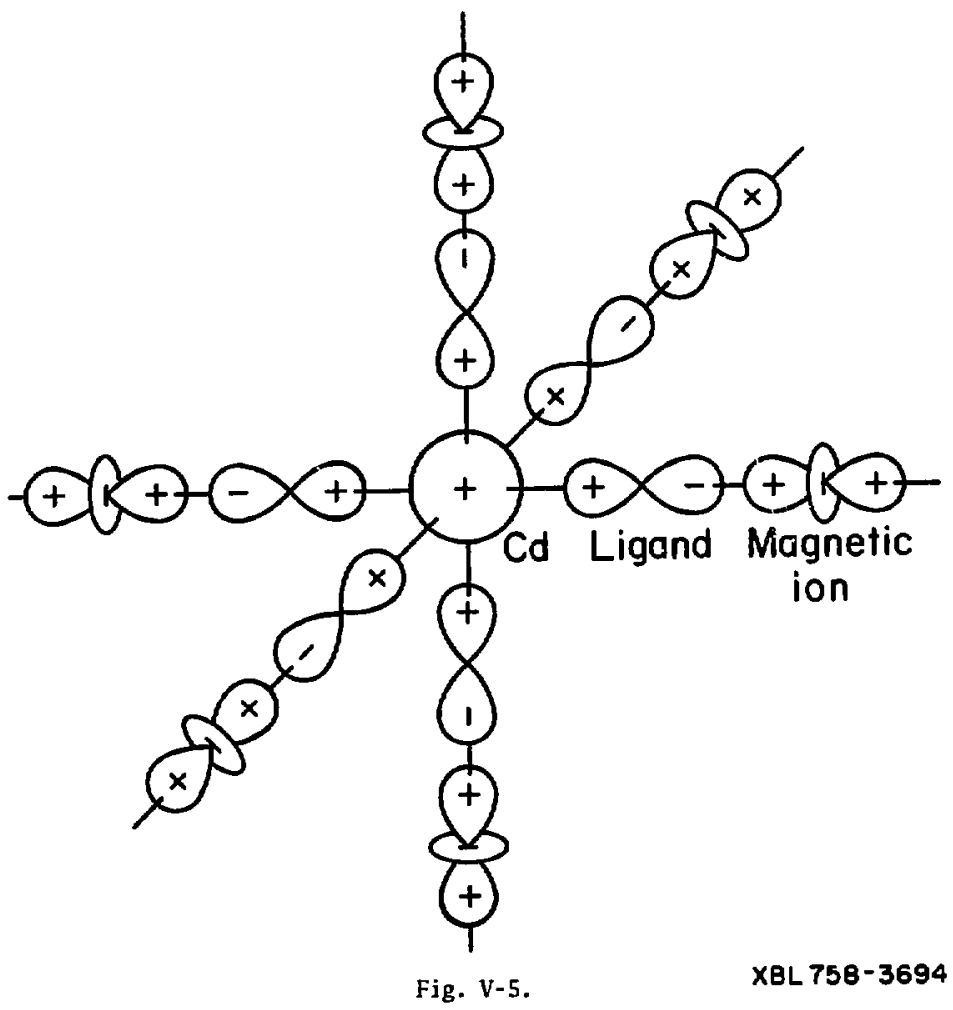


event was routed differently, and the PI and $E_{T}$ signals for both telescopes plus the TAC signal were sent to the computer. In other cases only th: singles $P I$ and $E_{T}$ were accepted.

A block diagram of the ${ }^{15_{B}}$ experiment electronics is shown in Fig. 9. Both the $\triangle E 2$ and recoil detectors were equipped with dual output fastslow preanplifiers. The slow output was used as the energy signal and the fast output to derive a timing signal for time of flight informacion. Amplified fast signals had a rise time of about 1 to $4 \mathrm{nsec}$, depending on the capacitance of the detector used. To minimize time walk, the timing signal was generated by a constant fraction discrininator (CFD), whose output is independent of pulse amplitude. The $\triangle E 2$ timing signal drove the start of a TAC, and the recoil timing signal, suitably delayed, provided the stop. The TAC output signal is thus the difference in time of flight ( $\triangle T D F)$ from target to detector between the exit particle and its recoil. As was done in the ${ }^{10} \mathrm{Li}$ experiment, the parameters (PI, $E_{\mathrm{T}}, \triangle \mathrm{E} 2$ and $\triangle E I$ in this case) for three-counter telescope events were always sent to the computer. In addition, the E-recoil and $\triangle T O F$ signals were taken when the event was a ${ }^{8} \mathrm{~B}$ with a recoil coincidence, and the event was then routed differently. For typicar counting rates of $10^{4}$ events/sec in the $\triangle E 2$ counter, $1^{8} \mathrm{~B}$ event/5 sec was identified, about 18 of these ${ }^{8} \mathrm{~B}$ events with a recoil coincidence.

H. Data Acquisition and Analysis

Events were sent from the ADC to an on-1ine PDP-5 computer and written on magnetic tape in unsorted form. The $\mathrm{E}_{\mathrm{T}}$ and PI parameters for 
Table II . Cadmium-Ligand Overlap Integrals
A. Fluorides

$$
\begin{array}{ll}
\mathrm{KNiF}_{3} & \mathrm{Ni}-\mathrm{F}=2.01 \mathrm{~A} \\
\mathrm{KCdF}_{3} & \mathrm{Cd}-\mathrm{F}=2.15 \mathrm{~A}
\end{array}
$$$$
\langle\mathrm{Cd} n \mathrm{~ns}| \mathrm{F}^{-} 2 \mathrm{po} \text { ) }
$$

$$
\mathrm{Cd}-\mathrm{F}=2.02 \mathrm{~A}
$$

(is $\mid 2 \mathrm{po}$ )

0.000752

(2s|2fo)

0.004965

(3s $2 \mathrm{p} \sigma$

0.019474

(4s 12po;

(ss $12 \mathrm{po})$

0.080115

0.191036

B. Oxides

$$
\begin{aligned}
& \text { NiO } \quad \mathrm{Ni}-\mathrm{O}=2.09 \mathrm{\AA} \\
& \mathrm{CdO} \quad \mathrm{Cd}-\mathrm{O}=2.34 \mathrm{~A} \\
& \langle\mathrm{Cd} n s| \mathrm{O}^{2-} 2 \mathrm{p} \phi
\end{aligned}
$$
$C d-0=2.09 R$
is $12 \mathrm{po}$
0.000796
$\mathrm{Cd}-0=2.34 \AA$
2s $\mid 2 p \sigma^{\circ}$
0.005262
0.000463
$3 \mathrm{~s} \mid 2 \mathrm{po}$
0.020661
0.003183
$4 s \mid 2 \mathrm{po}$.
0.084613
$0.01257 \mathrm{~s}$
$5 s \mid 2 p o$
0.209600
0.053453
0.194483 
$-44$.

Table II. (continued)

C. Chlorides

(Is 3po)

(2s 3pc)

$3 \mathrm{~s} 3 \mathrm{po}$ ?

4s 3pol

'5s 3po)
$\mathrm{Rb}_{2} \mathrm{CdCl}_{4}$
$\mathrm{Cd}-\mathrm{Cl}=2.60 \AA$

$$
\begin{gathered}
\left\langle\mathrm{Cd} n s \mid \mathrm{Cl}^{-}{ }^{-} \mathrm{po}\right\rangle \\
\mathrm{Cd}-\mathrm{Cl}=2.60 \AA \\
0.000667 \\
0.004420 \\
0.017280 \\
0.070140 \\
0.173920
\end{gathered}
$$


Table III. Metal-Ligand Overlaps
$\left\langle\mathrm{Mn}^{2+} \mathrm{d}_{2} 2 \mid \mathrm{F}^{-2} \mathrm{po}_{\mathrm{O}}\right\rangle=-0.0688$
$\left(\mathrm{MhrF}_{3}\right)$
$\left\langle\mathrm{Mn}^{2+} \mathrm{d}_{2} 210^{2-} 2 \mathrm{po}\right\rangle=-0.0712$
(Mn)
$\left\langle\mathrm{Mn}^{2+} \mathrm{d}_{\mathrm{z}}{ }^{2} \mid \mathrm{Cl}^{-}{ }^{-} \mathrm{pO}\right\rangle=-0.06117$
$\left(\mathrm{Rb}_{2} \mathrm{M} \mathrm{C1}_{4}\right)$
$\left\langle\mathrm{Co}^{2+} \mathrm{d}_{2} 2 \mid \mathrm{F}^{-2} \mathrm{po}\right\rangle=-0.0638$
$\left(\mathrm{KCOF}_{3}\right)$
$\left\langle\mathrm{Co}^{2+} \mathrm{d}_{\mathrm{z}^{2}} \mid \mathrm{0}^{2-} 2 \mathrm{Fr}\right\rangle=-0.0657$
$(\mathrm{C} \infty)$
$\left\langle\mathrm{Ni}^{2+} \mathrm{d}_{\mathrm{z}^{2}} \mid \mathrm{F}^{-} 2 \mathrm{po}\right\rangle=-0.0602$
$\left\langle\mathrm{Ni}^{2+} \mathrm{d}_{2} 2 \mid \mathrm{O}^{2-}{ }_{2 \mathrm{pO}}\right\rangle=-0.0621$
$\left(\mathrm{KNiF}_{3}\right)$
(NiO) 
Table IV.

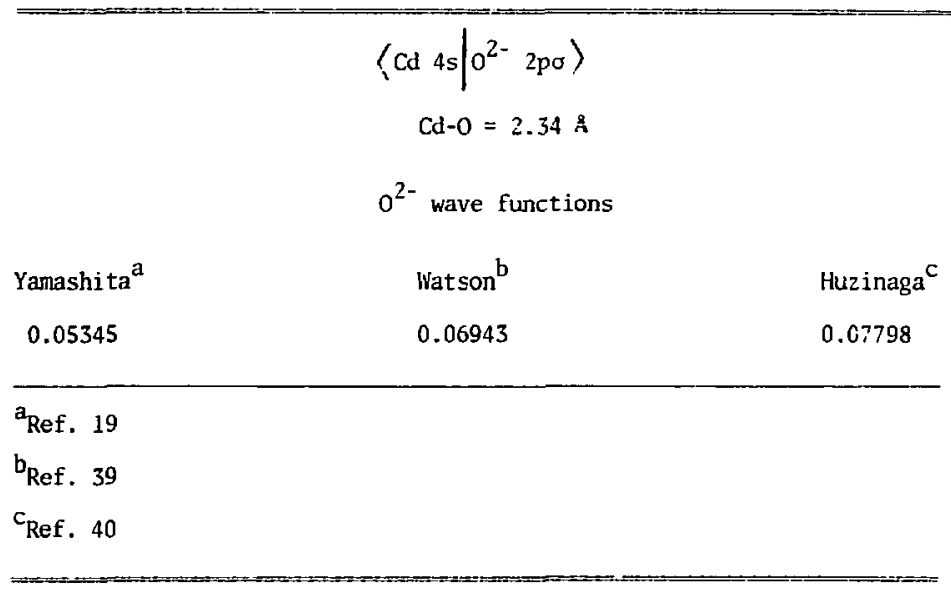


A limitation inherent in working with impurity doped samples is that the exact Cd-1igand bond distance is not known in the compounds. We have considered the two extremes possible, i.e. Cd-ligand distances representative of Cd compounds which are crystallographically isomorphous with the magnetic host materials, and the case where the Cd-1igand distance is taken equal to the metal-ligand distance of the magnetic host. A calculation of the hyperfine field for $\mathrm{KNiF}_{3}$ for which $\mathrm{f}_{\alpha}$ is known from $\mathbb{M R}^{21}$ is shown in Table V. Overlaps were computed for the bond distances characteristic of $\mathrm{Cd}-\mathrm{F}$ in $\mathrm{KCdF}_{3}$ and for the $\mathrm{Cd}-\mathrm{F}$ distance equal to the Ni-F separation. An intermediate Cd-ligand distance is probably the most realistic assimption. Even with the "short" bond distance the field calculated using all the Cd ns shells is lower than the experimental value by $20 \%$, and for the "long" bond length the calculated field comprises only $60 \%$ of the measured field. One also notes that the calculated .ield does not depend strongly ${ }^{14}$ on the Cd 5 s occupation due to the dependence of the nomalization constants on $a_{5 s}$. This means that the spin density parameters are relatively insensitive to direct meta $1 \mathrm{jd}$-Cd $5 \mathrm{~s}$ transfer, which we have neglected. The oxide data previously reported 4 support this contention, since from Table IA we see that the oxide fields are all con ${ }_{1}$ arable, even though Lau and Newnan $^{41}$ have calculated that in pure tho direct $3 \mathrm{~d}-4 \mathrm{~s}$ transfer will dominante the transferred hyperfine interactions.

In view of the limitations inherent in using Eq. 9 to predict hyperfine fields and thus spin-density parameters, we will normalize our data to $\mathrm{KNiF}_{3}\left(r_{0}=3.8^{2}{ }^{21}\right)$ and make estimates for other compounds relative to this reference point. 
Table V. Calculated $\mathrm{H}_{\mathrm{hf}}$ for $\mathrm{KNiF}_{3} \mathrm{f}_{\sigma}=3.8 \%$

\begin{tabular}{|c|c|c|c|c|}
\hline & \multicolumn{3}{|c|}{ Cd 5 s Occupation } & \multirow{2}{*}{$\begin{array}{c}\text { Experimenta } \\
\text { Field }\end{array}$} \\
\hline & $0 \%$ & $20 \%$ & $40 \%$ & \\
\hline $\mathrm{Cd}-\mathrm{F}=2.01 \AA(\mathrm{Ni}-\mathrm{F})$ & $62 \mathrm{KOe}$ & $84 \mathrm{KOe}$ & $79 \mathrm{KOe}$ & $105 \mathrm{KOe}$ \\
\hline $\mathrm{Cd}-\mathrm{F}=2.15 \AA\left(\mathrm{KCdF}_{3}\right)$ & $40 \mathrm{KOe}$ & $66 \mathrm{KOe}$ & $66 \mathrm{KOe}$ & $105 \mathrm{KOe}$ \\
\hline
\end{tabular}


Sawatzky's MO formulation provides a basis for the way in which the hyperfine field is expected to scale with $f_{\sigma}$. Figure 6 shows the results of a calculation (using parameters appropriate to $\mathrm{NiO}$ ) in which the Cd parameters were held fixed and $f_{\sigma}$ allowed to vary. One can see that the functional relationship connecting $f_{\sigma}$ to the hyperfine field is not as simple as a linear approxintation, but we will show that the assumption of a linear scaling will provide a reasonable lower bound to the estimated $f_{\sigma}$ values. $\mathrm{MR}^{21}$ and $E N D O R^{24}$ results are available for $\mathrm{Ni}^{2+}$ in fluorides and oxides, yielding $\mathrm{f}_{\sigma}=3.8 \%$ and $8.5 \%$ respectively. By adopting the figure of $3.8 \%$ and scaling the hyperfine fields for $\mathrm{NiO}$ and $\mathrm{KNiF}_{3}$ found in Table IA we would estimate $f_{\sigma}=7 \%$ in NiO. In Fig. 6 the calculated fields for $f_{\sigma}=4 \%$ and $8 \%$ were 55.3 and $99.7 \mathrm{KOe}$, and their ratio is 1.8 rather than the $2.0(8 \% / 4 \%)$ expected from the $f_{\sigma}$ values. Thus we see that the use of a linear approximation yields estimates of order $10 \%$ low because of curvature in the $\mathrm{H}_{\mathrm{hf}}$ vs $f_{\sigma}$ curve, but that the estimate of $7 \%$ is within about $20 \%$ of the ENDOR result and will be systematically low when generated by a ratio of fields. More quantitative studies ${ }^{4}$ have taken this curvature correction into account in order to obtain more precise values for the $f_{\sigma}$ parameters in the oxide saries. In this paper however, we are more concerned with elucidating the systematic crends in the data and will thus conteit ourselves with estimates which represent lower bounds.

The comparison of fluoride, oxide, chloride and sulfide measurements necessitates dropping the constraint that the Cd-1igand paraneters be held fixed as they were for the field calculations of Table $V$. To estimate the effect of changing the Cd-ligand properties, we have 


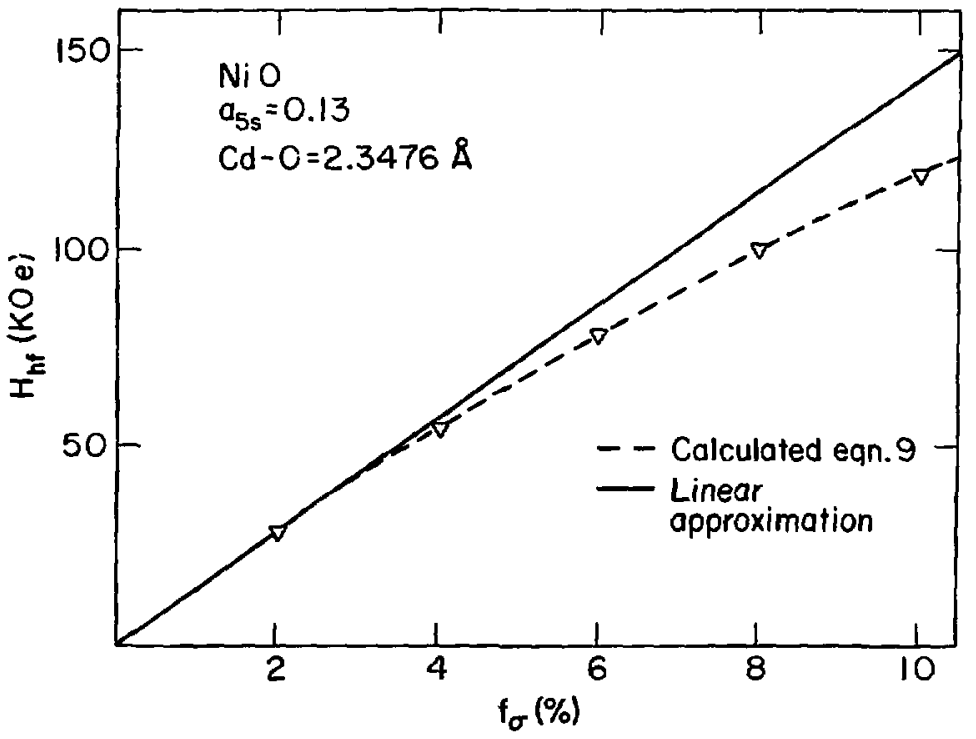

Fig. V-6.

XBL 758-3698 
calculated the ratio $\mathrm{H}_{h f}(\mathrm{Mn}-\mathrm{L}) / \mathrm{H}_{h f}(\mathrm{Mn}-\mathrm{F})$ witlı $f_{\sigma}$ held constant. The overlap integrals were those characteristic for Cd-L bond distances in the compounds $\mathrm{KCdF}_{3}, \mathrm{CdO}$, and $\mathrm{Rb}_{2} \mathrm{CdCl}_{4}$. In order to generate relative Cd 5 s occupations for the various 1igands, we plotted $1 / R_{C d-L}$ versus the empirical covalency parameter $\left(C=1-0.16\left|x_{m}-x_{L}\right|-0.035\left|x_{m}-x_{L}\right|^{2}\right)$ found by Hannay and Smyth, ${ }^{22}$ and found the approximately linear relation shown in Fig. 7. (The metal and ligand electronegativities were taken from the paper of Gordy and Thomas.) $)^{23}$ For $\mathrm{KNiF}_{3}$, we have $f_{\sigma}=(\gamma+\mathrm{S})^{2}$ $=3.8 \%$, and a calculation of the overlap contribution yields for $\gamma_{\mathrm{Ni}-\mathrm{F}} \sim 0.135$. A Cd-F $\gamma$ value was generated by scaling the covalency farameters for Cd-F/Ni-F. This yields $\gamma_{\mathrm{Cd}-\mathrm{F}} \sim 0.106$. The $\mathrm{a}_{5 \mathrm{~s}}$ parameter was then adjusted to yield a Cd-F 5s occupancy of 10.6\%. The other ligand Ss occupancies were scaled by the linear relationship of Fig. 7. The result of the calculation for $f_{\sigma}$ fixed at $8 \%$ is

$$
\frac{\mathrm{H}_{h f^{(\mathrm{Mn}-\mathrm{L})}}}{\mathrm{H}_{\mathrm{hf}}\left(\mathrm{hrn}_{\mathrm{n}-\mathrm{F})}\right.}=1.0 \pm 0.1 \mathrm{~L}=\mathrm{F}^{-}, \mathrm{O}^{2-} \text {, and } \mathrm{Cl}^{-} \text {. }
$$

The total spread in the relative fields with $f_{\sigma}$ held fixed is only of order $10 \%$ and indicates that the magnitude of the experimental fields are not grossly sensitive to a consistent set of $\mathrm{Cd}$-ligand parameters. By a consistent set of parameters we mean Cd-ligand bond distances which are either all "long" or all "short" with their associa:ed overlaps. Thus major changes in measured hyperfine fields are to be primarily associated in changes in $f_{o}$ and a simple ratio of measured fields should provide a reasonable lower bound to the spin density parameters. 


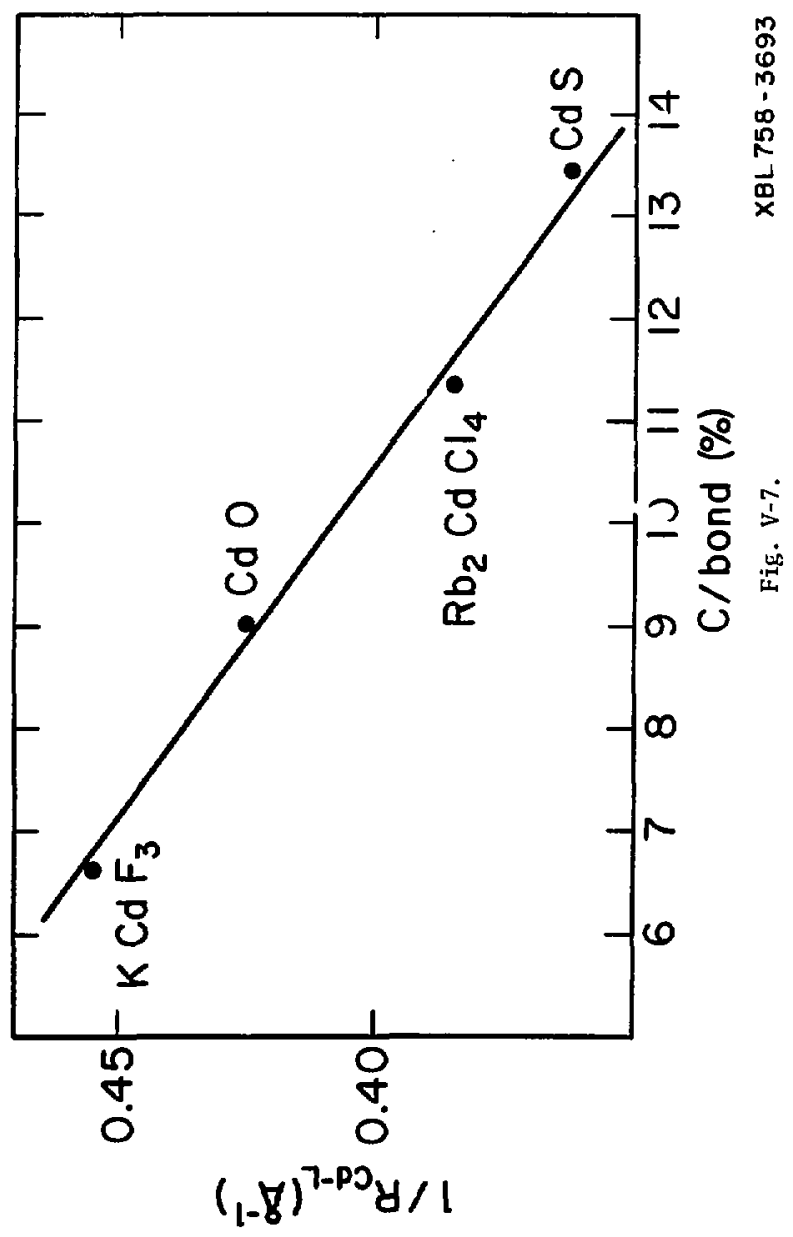


D. SYSTEMATICS OF THE Cd HYPERFINE FIELDS AND SPIN DENSITY PARAMETERS Before starting an analysis of the data in Tables IA and IB, it is necessary to digress and explain how the fields listed in the column $\mathrm{H}_{\mathrm{hf}}$ were arrived at. The reason that the hyperfine fields differ by more than a normal dipolar contribution from the experimental values is due to zero point spin deviations in Heisenberg-1ike antiferromagnets. 25 The necessity for this correction arises because of the term $\frac{\left\langle s_{z}\right\rangle}{S}$ (Eq. 9). The deviation of $\left\langle S_{2}\right\rangle$ from its full value in these high-spin complexes depends on the dimensionality of the magnetic lattice. For that reason we have separated the host materials into two- and threedimensional categories. Note that the co compounds $\mathrm{K}_{2} \mathrm{CoF}_{4}$ and $\mathrm{Rb}_{2} \mathrm{CoF}_{4}$ are Ising-like, ${ }^{26}$ and should therefore not dispisy any spin deviations. We assume that the ful 1 theoretical ${ }^{25}$ spin devirtion is present for both the magnetically two- and three-dinensional Heisenberg-like compounds. In this case

$$
\left.\frac{\left\langle S_{z}\right\rangle}{S}\right)_{2,3}=1-\frac{\Delta_{2,3}}{S} \text {, where } \Delta_{2}=0.197 \text { and } \Delta_{3}=0.078 \text {. }
$$

Thus the spin deviations for $\mathrm{Ni}^{2+}\left(\mathrm{S}^{=1}\right)$ are much larger than for $\mathrm{Mn}^{2+}(\mathrm{S}=5 / 2)$ and vary with the magnetic dimensionality. The hyperfine field is then related to the experimentally measured value by

$$
H_{h f}=H_{\exp ^{\prime}} / 1-\Delta / S \pm H_{d i p}
$$

where an additional (and usually small) dipolar field correction has also been made whenever the relative sign of the dipolar and supertransferred ficlds is known. 
We wish to examine the systematic trends of the hyperfine fields for $\mathrm{Cd}$ doped into various host antiferromagnets. We will begin by considering the effect of moving across the transition metal grow for a fixed ligand. For the fluorides the data allow the following comparisons.

$$
\begin{aligned}
\mathrm{MnF}_{3}(124.4) & \mathrm{KCoF}_{3}(78.2) \\
\mathrm{RbMnF}_{3}(117.4) \rightarrow \mathrm{KFeF}_{3}(104.2) \rightarrow & \left.\mathrm{RbCoF}_{3}(71.5) \rightarrow \mathrm{KNiF}_{3}(113.7)+\mathrm{KCuF}_{3} i<4\right) \\
\operatorname{TuMnF}_{3}(122.5) & \mathrm{TLCoF}_{3}(71.6)
\end{aligned}
$$

and

$$
\underset{\mathrm{Rb}_{2} \mathrm{MnF}_{4}(75.4)}{\mathrm{K}_{2} \mathrm{MnF}}{ }_{4}^{(79.4)} \underset{\mathrm{Rb}_{2} \mathrm{CoF}_{4}(50.0)}{\mathrm{K}_{2} \mathrm{CoF}_{4}(52.7)} \rightarrow \mathrm{Rb}_{2} \mathrm{NiF}_{4}(64.7)
$$

Several coments are in order concerning some of the field values. of particular interest are the consistently low $\mathrm{Co}^{2+}$ values, which we attribute to the fact that the orbital angular momentum is not quenched in these compounds. This is also expected to be present to some degree in the $\mathrm{Fe}^{2+}$ compound, but the effective reduction of the spin expectation value is clearly not as large. In addition, $\mathrm{MWrF}_{3}{ }^{27,28}$ and $\mathrm{KFeF}_{3}{ }^{29}$ both have lattice distortions and canted spin structures at liquid helium temperatures. The field for $\mathrm{MnF}_{3}$ is a rougis estimate based on assuming that

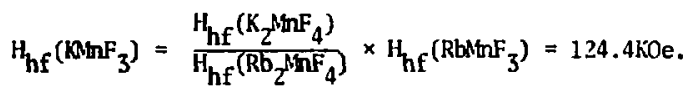

No at termpt was made to estimate a field for "uncanted" $\mathrm{KFeF}_{3}$. The hyperfine fields for these compounds should therefore be considered as only approximate. 
The $\mathrm{KCuF}_{3}$ is a special case which reflects the strong Jahn-Teller effect and the A type magnetic structure of the compound. The Jahn-Teller effect leads to an orthorhombic disiortion of the $\left(\mathrm{CuF}_{6}\right)^{4-}$ octahedron with two short and two long Cu-F bonds in the (001) plane and a mediun Cu-F separation for the two anions along the c-axis ${ }^{30}$ (see Fig. 8). The $\mathrm{Cu}^{2+}$ ground state $\left(\mathrm{x}^{2}-y^{2}\right)$ and $\mathrm{F}^{-} 2 \mathrm{po}$ wavefunctions are arranged such that in the (001) plane unpaired spin is approximately transferred only from the two neighboring magnetic ions along the c-axis and from the Cu's with short Cu-F bonds. Spins in adjacent (001) planes are coupled antiferromagnetically however, so that the transferred spin densities are antiparallel and expected to cancel at the Ca rucleus. This is consistent with our observation that $\mathrm{H}_{\mathrm{hf}} \leq 4 \mathrm{KOe}$.

The general trend in the fluorides with perovskite or perovskitelike stru:tures in moving across the 3d metal group is that the fields are very nearly equal in mignitude, with the Co values systematically low. The same trends sre present for the oxide series with NaCl structure,

$$
\mathrm{NhO}(208) \rightarrow \mathrm{CoO}(187)+\mathrm{NiO}(212)
$$

the virtual constancy of the fields for a fixed ligand and crystal structure is expected on a chemical basis. The electron affinities for the $3 \mathrm{~d}$ transition metals are comparable $\left(\mathrm{Mn}^{2+}\right.$ is slight $1 \mathrm{y}$ : $\left.\mathrm{OW}\right)$ and the bond distances are quite similar. Thus one expects that the spin density parameter $f_{\sigma}$, which has both covalint and overlap components, will not alter drastically across the transition metal group under the constraint of fixed ligand and crystal structure. Ir. order to estimate spin density parameters for our data, we will make the assumption outlined in 


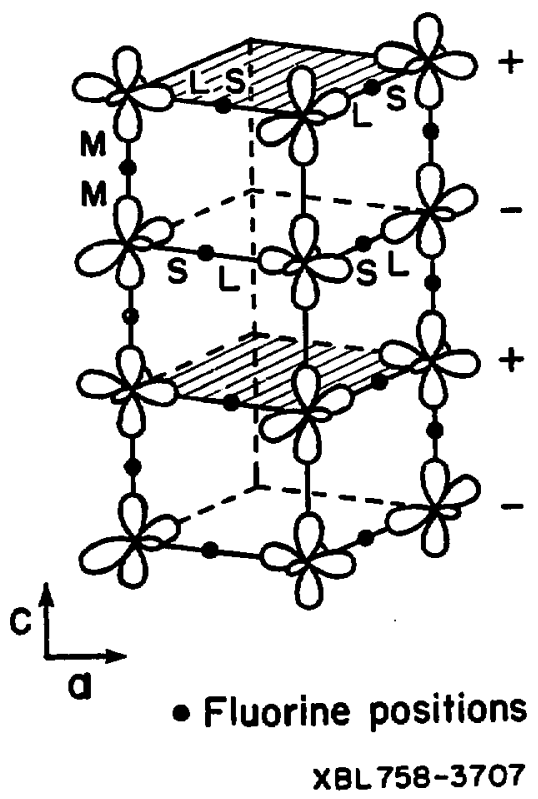

Fid. V-8. 
Section III that $f_{\sigma}$ scales linearly witn $H_{h f}$, and will use the value for $\mathrm{MMiF}_{3}\left(f_{\sigma}=3.8^{\circ}\right)$ as a nomalization point. The compounds of form $\mathrm{A}_{2} \mathrm{MX}_{4}$ have only 4 magnetic spins contributing to the transferred $\mathrm{Cd}$ field, so that field value for $\mathrm{KNiF}_{3}(113.7 \mathrm{kOe})$ was reduced by $4 / 6$ before making a spin-density estimate. The $f_{\sigma}$ results for our PAC data are shown in Table 6, along with results of other techniques.

A few additional points concerning the PAC estimated fluoride and oxide spin density parameters are worth noting. First of all, $\mathrm{Nm}{ }^{2+}$ does not appear to be anomalous in either fluoride or oxide series. Particularly in the fluorides, a value of approximately $4:$ is consistent with the basic trend that the expected order of magnitude is 3-5\%. Even though our estimate for $\mathrm{NiO}$ is lower by $20 \%$ than the ENDOR result, the near constancy of the measured fields indicates that the oxides, like the fluorides, have essentially similar $f_{\sigma}$ values. We thus feel that a lower bound of $7 \%$ for $\mathrm{N}$ ho is quite reasonable and contend that nothing anomalous happens in passing along the $3 d$ group metals in isomorphous salts. Based on our normalization to MR fluoride data, the reasonable agreement with the ENDOR medsurement for $\mathrm{Ni}^{2+}(\mathrm{MgO})$ argues for the basic equivalence of data measured in diluted and concentrated magnetic systems. The Co data consistently show the effects of unquenched orbital angular momentum in all the various techniques.

We now turn to the trends expected when the $3 \mathrm{~d}$ metal is held fixed and the ligands varied. Only Mn compounds exist in magnetic structures which can be compared for all the ligands $\mathrm{F}^{-}, \mathrm{O}^{2-}, \mathrm{Cl}^{-}$, and $\mathrm{S}^{2-}$. In order (1) incorporate all rour ligands it is ncessary to use data from the

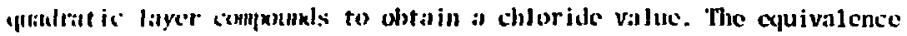


Tahle o, $r$ vatues derived fma Mu:

\begin{tabular}{|c|c|c|c|}
\hline $\mathrm{AN}^{24}$ & $\Gamma \mathrm{c}^{2+}$ & $-\cos ^{2 *}$ & $\mathrm{Ni}^{2+}$ \\
\hline $\operatorname{BC}\left(\mathrm{r}_{0}\right)$ & PM $\left(f_{a}\right)$ & PiKC $\left(f_{0}\right)$ & $\ln \left(\mathrm{f}_{\mathrm{f}}\right)$ \\
\hline 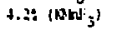 & s.s: $\left(\mathrm{AFer}_{3}\right)$ & $2.6:\left(\mathrm{NCOF}_{3}\right)$ & B. B: $\left(\mathrm{N}_{3} \mathrm{~F}_{3}\right)$ \\
\hline 3.94 (Rhinf 3 ) & & $2.45\left(\mathrm{RbCOF}_{3}\right)$ & $3.59\left(\mathrm{~K}_{2} \mathrm{NifF}\right)$ \\
\hline $4.11\left(T W_{h} f_{3}\right)$ & v & $2.48\left(\mathrm{TICOF}_{3}\right)$ & $3.24\left(\mathrm{Fb}^{2} \mathrm{NiF_{4 }}\right)$ \\
\hline $4.02\left(K_{2}+F_{4}\right)$ & & $2.06\left(X_{2} \operatorname{CoF}_{4}\right)$ & \\
\hline 3. BI $\left(\mathrm{Rb}_{2} \mathrm{hnF}_{4}\right)$ & & $\left.2.51\left(\mathrm{Rb}_{2} \operatorname{CoF}\right)_{4}\right)$ & \\
\hline MPVIESR $\left\{f_{0}-f_{n}\right\}$ & & $\operatorname{ESR} \frac{\left(f_{3}-f_{n}\right)}{2}$ & WW/ESR $\left(f_{\mathrm{o}}\right)$ \\
\hline $0.21\left(\mathrm{WhF}_{3}\right)^{2}$ & & & $3.84\left(\mathrm{KYiF}_{3}\right)^{\mathrm{e}}$ \\
\hline $0.31\left(\mathrm{Ab}_{\mathrm{n}} \mathrm{fF}_{3}\right)^{b}$ & & $2.45\left(\mathrm{D}_{4} \mathrm{~F}_{3}\right)^{\mathrm{d}}$ & $3.2:\left(12+8 F_{3}\right)^{E}$ \\
\hline $0.3:\left(\mathrm{NHFF}_{3}\right)^{\mathrm{C}}$ & & assunc $f_{0}-f_{11}$ & \\
\hline & & estimate $f_{0}-2.48$ & $D\left(f_{a}+f_{s}\right)$ \\
\hline & & & $258\left(\mathrm{NILF}_{3}\right)^{\mathrm{g}}$ \\
\hline & & & $t_{5}=0.51$ \\
\hline & & & estinate $f_{0}-2.31$ \\
\hline
\end{tabular}

\section{Onides}

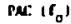

7.03 (Mn)

$\operatorname{orc} \mathrm{c}_{\mathrm{a}} \cdot 3 \mathrm{f}_{\mathrm{a}} \cdot\left(\mathrm{f}_{\mathrm{s}}\right)^{\mathrm{h}}$

$3.63(\mathrm{HD})$

$f_{\sigma}=f_{\pi}, f_{s}=0.6$

est Lase $f_{\alpha}-1.51$

aton $\left(f_{0}-f_{1}\right)^{2}$

$0.85(100)$

$$
\operatorname{PAr}\left(E_{\mathrm{a}}\right)
$$

S.4: $\left.10, \mathrm{HnCl}_{4}\right)$
PYC $\left(E_{g}\right)$

$0.28(C 0)$

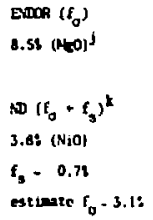

Chlorides

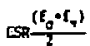$$
\sin \left(\cos _{0}\right)^{-4}
$$

\section{sulfide}

$$
\text { PAC ( } \left.0_{0}\right)
$$

B.6: $(2+$ this $)$

$$
\begin{aligned}
& \text { Ant } f_{7}+2 f^{n}+f^{3}, \\
& \text { ?.n: }(1,405)^{m}
\end{aligned}
$$

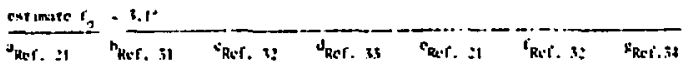

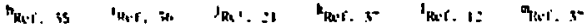


of $f_{\sigma}$ values between the perovskites and quadratic-1.ayer compounds $\mathrm{KnFF}_{3} / \mathrm{K}_{2} \mathrm{MnF}_{4}, \mathrm{RbMnF}_{3} / \mathrm{Rb}_{2} \mathrm{MnF}_{4}, \mathrm{KCoF}_{3} / \mathrm{K}_{2} \mathrm{CoF}_{4}, \mathrm{RbCoF}_{3} / \mathrm{Rb}_{2} \mathrm{CoF}_{4}$ and $\mathrm{KNiF}_{3} / \mathrm{K}_{2} \mathrm{NiF}_{4}$ when adjustments for spin deviations and number of magnetic neighbors are made illustrates that the quadratic-layer compounds yield $f_{\sigma} \quad$ estimates consistent with a perovskite-like magnetic sublattice. The hyperfine fields in MnO and ins also derive from a perovskite-like magnetic lattice ${ }^{4}$ so we will consider the series $\mathrm{Rb}_{2} \mathrm{MnF}_{4}$, $\mathrm{MnO}, \mathrm{Rb}_{2} \mathrm{MnCl}_{4}$ and ins.

One expects a weak dependence on the Cd-1igand parameters and a systematic increase in observed hyperfine field on traversing tie series $\mathrm{F}^{*}, \mathrm{O}^{2-}, \mathrm{Cl}^{-}$and $\mathrm{S}^{2-}$ in the direction of increasing covalency. Table VII shows that the measured fields for the chalcogenides $\mathrm{MnO}$ and $\mathrm{AnS}$ and their derived spin density parameters are not consistent with this 1 rend. We suggest that the answer is reflected in the graph of Fig. 9, in which we have separated the halides and the chalcogenides and plotted the derived $f_{\sigma}$ values versus the covalency parameter of Hannay and Smyth. ${ }^{22}$ For all four cempounds the magnetic sublattice contributing to the observed fields are equivalent, but there is a decided difference between the perovskite-1ike $\mathrm{K}_{2} \mathrm{NiF}_{1}$ structure and the $\mathrm{NaCl}$ structure of MnO and Mns. In the first cisse the fluori le and chloride ligands are coordinated to only two magnetic neighbors (pure host) while in the $\mathrm{NaCl}$ structure the ligands are attached to six magnetic neighhors. The spin structure in MnO and MnS is sticn that most of these moments cir'icel at ${ }^{1} \mathrm{Cd}$ impurity by symuetry, ${ }^{4}$ but the charge pulling effect on tho lifind is still mesent. No sugpent that this is the reason why the 
Table VII

Magnetic Host $\quad \begin{gathered}\mathrm{H}_{\mathrm{hf}} \\ \text { (KOe) }\end{gathered}$

$\mathrm{Rb}_{2} \mathrm{NenF}_{4}$

$3.8 \mathrm{o}$

$\mathrm{Rb}_{2} \mathrm{MnCl}_{4}$

160

$5.4 \%$

Mno

208

$7.0 \frac{8}{6}$

$\alpha-\operatorname{sins}$

258

$8.6 \%$ 


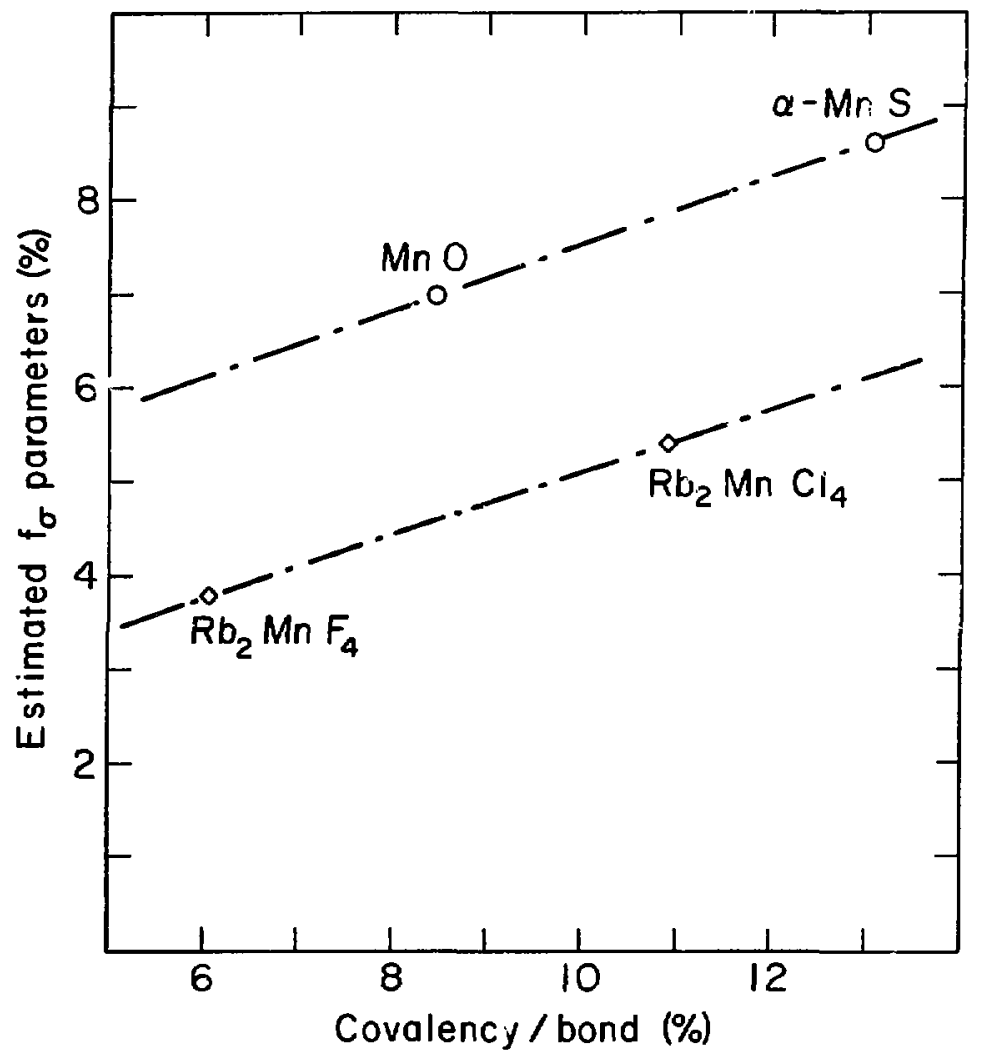

Fig. $v-9$. 
chalcogenides have fields of order 200-250 kOe and the "adjusted" quadratic-layer halides fields of 120-160 KOe. Even though only two points exist on each "curve", we feel that the plot of Fig. 9 is fairly indicative of the anticipated increase in $f_{\sigma}$ due to increasing anion covalency.

E. CONCLUSION

We have applied a simple model to the estimation of spin density parameters derived from PAC data and find the expected systematic trends both along a group and down a column. The data indicate that spin density parameters derived from concentrated and dilute samples are approximately equal, and that increasing ligand covalency is reflected in increasing values of the spin density parameters. We also conclude that Non does not act anomalously in either in fluoride or oxide series and we estimate $f_{\sigma}$ values for $\mathrm{Mn}^{2+}$ in the perovskite-like fluorides of $4 \%$ and values of order $7-8 \%$ for the oxides. 
RLFERENCES

1. J. B. Goodenough and J. M. Longo Landolt-Börnstein III/4a, Magnetic Oxides and Related Compounds

$$
\begin{aligned}
& \text { Springer-Verlag, Berlin-Heidelberg- } \\
& \text { New York } 1970 \text { p: 126-275. }
\end{aligned}
$$

2. H. H. Rinneberg and D. A. Shirley $\left(\mathrm{RbMhF}_{3}\right.$ paper) Phys. Rev. Lett. 30 (22), 1147 (1973).

3. H. Haas and D. A. Shirley, LBL-1284 (1972). Submitted to Journal of Chemical Physics.

4. H. H. Rinneberg and D. A. Shirley (NiO paper) LBL-2900 (1974). Submitted to Phys. Rev. B.

5. B. Erik Karlsson, Arkiv, Fysik 22, 1 (1962).

6. Handbook of Preparative Inorganic Chemistry V2, Edited by Georg Brauer, p. 1465, Academic Press, New York, London 1965.

7. Perturbed Angular Correlations, Edited by E. Karlsson, E. Mathias, and K. Siegbahn, North Holland Pub. Co.-Amsterdan (1964).

8. H. H. Rinneberg and D. A. Shirley - difluoride paper, LBL-2398 (1974). Submitted to Phys. Rev. B.

9. J. H. Van Vleck, Phys. Rev. 41, 208 (1932).

10. J. S. Griffith "The Theory of Transition Metal Ions" Cambridge University Press, London (1961).

11. C. J. Bollhausen "Introduction to Ligand Field Theory" McGraw-Hill, New York (1962).

12. J. Owen and J. H. N. Thornley, Rep. Prog. Phys. 29, $675\left(19\left(i_{1}\right)\right.$.

13. .. A. Tossid, I. .I. Vaughiall and K. II. Johnson Nature 244, 42 (1973). 
14. F. van der Woude and G. A. Sawatzky, Phys. Rev. B. 4 (9) 3159 (1971). 15. J. M. D. Cocy and G. A. Sawatzky, J. Phys. C: Solid St. Phys. 4, 2386 (1971).

16. C. Boekmma, F. van der Woude, and G. A. Sawatzky, Int, J. Magnetism 3, 341 (1972).

17. J. B. Mann, Los Alamos Sci. Lab. Report LA-36́91 (1968).

18. E. Clementi, IBM J. Res. Developm. 9, 2 (1965).

19. J. Yamashita and S. Asano, J. Phys. Soc. Japan 28, 1143 (1970).

20. Y. Toyotomi and R. Onaka, J. Phys. Soc. Japan 34, 623 (1973).

21. R. G. Shulman and K. Knox, Phys. Rev. Lett. 4, 603 (1960).

22. N. B. Hannay and C. P. Smyth, J. An. Chem. Soc. 68, 171 (1946).

23. W. Gordy and W. J. O. Thomas, J. Chem. Physics 24, 439 (1956).

24. P. Freund, J. Phys. C: Sol id State Phys. 7, L33 (1974).

25. P. W. Anderson, Phys. Rev. 86, 694 (1952).

26. D. J. Breed, K. Gilijanse, and A. R. Miedema, Physica 45, 205 (1969).

27. A. J. Heeger, O. Beckman,, and A. M. Portis, Phys. Rev. 123, 1652 (1961).

28. K. Saiki, K. Horai, and H. Yoshioka, J. Phys. Soc. Japan 35, 1016 (1973).

29. G. R. Davidson, M. Eibschlltz, and H. J. Guggenhein, Phys. Rev. B $\underline{8}$, 1864 (1973).

30. S. Kadota. I. Yamada, S. Yoneyama, and K. Hlirakawa, J. Phys. Soc. Japan 23, 751 (1967).

31. M. B. Walker and R. W. II. Stevenson, Proc. Phys. Soc. 87, 35 (1966).

32. I. I'. I'. Ilall, W. Ilayers, R. W. II. Stevenson, and J. Wilhans, J. (hem. l'hys, 38, 1977 (1905), 39, 35 (196.3). 
33. J. H. M. Thornley, C. G. Windsor, and J. Owen, Proc. Roy. Soc. A 284,252 (1965).

34. M. T. Hutchings and H. J. Guggenheim, J. Phys. C: Solid State Phys. 3. 1303 (1970).

35. A. J. Jacobson, B. C. Tofield, and B. E. F. Fender, J. Phys. C: Solid State Phys, 6, 1615 (1973).

36. P. Freund, J. Owen, and B. Hann, J. Phys. C: Solid State Phys. $\underline{6}$, L139 (1973).

37. B. E. F. Fender, A. J. Jacobson, and F. A. Wedgwood, J. Chem. Phys. 48, 990 (1968).

38. CRC Handbook of Chemistry and Physics 53ril Edition, B202, 1972-1973. Chemical Rubber Company, Cleveland, 1972, R. C. Weast, Ed.

39. R. E. Watson, Phys. Rev. 111, 1108 (1958).

40. S. Huzinaga and A. Hart-Davis, Phys. Rev.Annal 8, 1734 (1973).

41. B. F. Lau and D. J. Newman, J. Phys, C: Solid State Phys. 6, 3245 (1973). 


\section{FIGURE CAPTIONS}

Fig. 1. Tine modulated perturbation factor for ${ }^{111} \mathrm{~m} \mathrm{Cd}$ in $\mathrm{KCuF}_{3}$ illustrating the effect of a non-zero asymmetry paraneter on the periodicity of the oscillations. The time calibration is 1.619 ns/channel .

Fig. 2. Time spectrum for ${ }^{111 \mathrm{~m}} \mathrm{Cd}$ in $\mathrm{K}_{2} \mathrm{NiF}_{4}$ at $4.2 \mathrm{~K}$ which displays the effects of a combined magnetic dipole-electric quadrupole interaction. The time calibration is $1.619 \mathrm{~ns} /$ channel.

Fig. 3. Power spectral density transform of the combined interaction time spectrun of ${ }^{111} \mathrm{~m} \mathrm{Cd}$ in $\mathrm{K}_{2} \mathrm{NiF}_{4}$ at $4.2^{\circ} \mathrm{K}$.

Fig. 4. Molecular orbital diagram for the $3 d$ transition metal salts.

Fig. 5. Schematic diagram of the extended octahedral complex relative to $\mathrm{Cd}$-doped host antiferromagnets with perovskite-like magnetic structures.

Fig. 6. P1ot of calculated hyperfine fields based on the extended cluster model versus spin density. The Cd parameters bave been held fixed in this calculation.

Fig. 7. Plot of $1 / R_{\mathrm{Cd}-\mathrm{Ligand}}$ versus the Cd-Ligand covalency/bond as defined by Hannay and Smyth.

Fig. 8. Crystallographic and spin structure of $\mathrm{KCuF}_{3}$ showing the halffilled Cu orbitals after Ref. 30. Another crystallographic modification exists.

Fig. 9. Plot of our estimated spin density parameters versus the MnLigand covalency. Two separate anticipated trends are shown for the chalcogenides ( $\mathrm{NaCl}$ structure) and the halides (perovskitelike structure). 
VI. MEASUREMENTS OF SOLID STATE PHENOMENA*

A. Measurement of Spin Deviations, Pressure Induced Hyperfine Field Shifts; and Sublattice Magnetizations Using Perturbed Angular Correlations

\section{Introduction}

TDPAC studies of metals and allcys have proliferated in recent years following the resurgence of theoretical interest in the slectric field gradients of pure metals. Applications in insulators remain minimal however, and have not tended to emphasize the fundamental properties of solids which are potentially available through measurements of magnetic and electric hyperfine interactions.

The purpose of this paper is to demonstrate that in a manner analogous to resonance methods, PAC techniques can be applied to probe a versatile range of solid state phenomena. Apropos to this endeavor, we have measured the deviation of the spin expectation value in magnetically two-dimensional Heisenberg-like antiferromagnets induced by quantum mechanical zero-point energy as wel: as the sublattice magnetization next to a diamagnetic impurity. Finally we report the first PAC measurenents in an insulator of the pressure induced shift of the supertransferred hyperfine field in Cddoped $\alpha$-MnS. Section 2 contains the experimental details, while Sections 3 and 4 treat the results and discussion of these experinents.

2. Experimental

a) Sample Preparation

The preparation of $\mathrm{Cd}$-doped $\mathrm{MnF}_{2}^{1}, \mathrm{RbNhF}_{3}^{2}, \mathrm{KNiF}_{3}^{2}$, and $\mathrm{K}_{2} \mathrm{NiF}_{4}^{3}$,

"This section of work was done in conjunction with H. H. Rinneberg and 1). A. Shirley. 
$\mathrm{Rb}_{2} \mathrm{Mnn} \mathrm{F}_{4}^{3}$, and $\alpha-\mathrm{Ans}^{3}$ has previously been reparted. The basic technique involves the themal diffusion of active ${ }^{11 \mathrm{~m}} \mathrm{CdF}_{2}$ into materials prepared according to the solid state reactions

$$
\begin{aligned}
& \mathrm{AX}+\mathrm{MX}_{2} \rightarrow \stackrel{\Delta}{\rightarrow} \mathrm{AMX}_{3}, \\
& \mathrm{ZAX}+\mathrm{MX}_{2} \rightarrow \mathrm{A}_{2} \mathrm{MX}_{4} .
\end{aligned}
$$

Since $\mathrm{KF}$ and $\mathrm{RbF}$ are both highly iygroscopic, it was necessary to work under dry conditions both in the preparation and doping of compounds reacted in the solid state.

Active $\alpha$-NhS was prepared by coprecipitation, dried, and then fired to $1000^{\circ} \mathrm{C}$ under a strean of dry $\mathrm{H}_{2} \mathrm{~S}$. The concentration of $\mathrm{Cd}$ dopant generally did not exceed 0.1 at .5 in aly of the compounds.

b) Equipment and Data Analysis

The measurements of PAC spectra taken on an 8 counter $\gamma^{-n}$ : spectrometer and the subsequent data analysis are described in an earlier paper. ${ }^{3}$

Spectra taken at liquid heliun or liquid nitrogen temperatures were obtained by imersing an aluminum sample holder directly into the refrigerant. For intemediate temperatures a regulator of the type described by Welber et al. ${ }^{4}$ was used. The temperature was measured using a $\mathrm{Au}(\mathrm{Fe}) / \mathrm{Fe}$ thermocuple which had been calibrated against a doped germanium thermometer. A stability of $\pm 0.2 \%$ in regulation was achieved over several hours for temperatures between 15 and $100^{\circ} \mathrm{K}$.

The pressure cell employed in this work is similar to that described by S. D. Bader ${ }^{5}$ except that the cell body was made out of A286 steel and four windows were partiallv machined through the body for easy $\gamma$-ray exit. 


\section{Results}

Time spectra recorded for Cd-doped $\alpha-\mathrm{AmS}$ are shown in Fig. 3. Characteristic spectra for perovskites ${ }^{2}\left(\mathrm{NNiF}_{3}, \mathrm{RbMnF}_{3}\right)$, quadratic-layered fluorides ${ }^{3}\left(\mathrm{~K}_{2} \mathrm{NiF}_{4}, \mathrm{~Pb}_{2} \mathrm{MnF}_{4}\right)$, and difluorides ${ }^{1}\left(\mathrm{MnF}_{2}\right)$ have previously been published. The modulation pattertis have been corrected for background and exponential nuclear decay, and all samples displayed structure consistent with single phase random polycrystals. Damping in $\alpha$-NhS under pressure was accounted for in the least squares fitting routine by intraducing a Gaussian distribution of fields.

Table I sumarizes the drta relevant to the spin expectation value experiments while Tables II $A, B$ and III list the measured parameters for the temperature dependent sublattice magnetization and pressure induced field shift studies.

\section{Discussion}

\section{a) Zero point spin deviations}

The problem of how the inciusion of quantum mechanical zero point energy affects the antiferromanetic ground state was treated as early as 1952 by P. W. Anderson. ${ }^{6}$ At that time he derived values for the expected spin deviation within the franework of spin wave theory and neglecting magnetic anisotropy. Of fundanental importance was the result that the expected magnitude of the spin deviation depended very strongly on the maguetic dimensionality of the lattice.

Considerable additional theoretical work ${ }^{7,8,9}$ has been done on this problem, but experimental confinmation was lacking until the late 1960's when it was recognized that $\mathrm{K}_{2} \mathrm{NiF}_{4}$ and $\mathrm{K}_{2} \mathrm{MnF}_{4}$ (and their $\mathrm{Rb}$ analogs) 
Table I.

\begin{tabular}{lccc}
\hline Host & $\begin{array}{c}\text { Meas. } \\
\left({ }^{\circ} \mathrm{K}\right.\end{array}$ & $\begin{array}{c}H\left(4.2^{\circ} \mathrm{K}\right) \\
(\mathrm{KDe})\end{array}$ & $\begin{array}{c}\mathrm{V}_{\mathrm{Q}}=\frac{3 \mathrm{e}^{2} \mathrm{gQ}}{20 \mathrm{~h}} \\
(\mathrm{NHz})\end{array}$ \\
\hline $\mathrm{NNiF}_{3}$ & 253 & $104.8 \pm 1.5$ & 0.0 \\
$\mathrm{RbMhF}_{3}$ & 82.9 & $113.8 \pm 1.5$ & 0.0 \\
$\mathrm{~K}_{2} \mathrm{NiF}_{4}$ & 97 & $54.9 \pm 1.5$ & $3.7 \pm 0.2$ \\
$\mathrm{Rb}_{2} \mathrm{NnF}_{4}$ & 38.5 & $67.9 \pm 1.5$ & $4.7 \pm 0.2$ \\
\hline
\end{tabular}


Table II.A.

\begin{tabular}{|c|c|c|}
\hline & $\begin{array}{c}\mathrm{MnF}_{2} \\
\mathrm{~T}_{\mathrm{n}}=66.9^{\circ} \mathrm{K} \\
v_{\mathrm{Q}}=16.5 \mathrm{MHz} \\
n=0.06\end{array}$ & \\
\hline$(x \pm 0.2)$ & & $\begin{array}{c}\mathrm{H}_{\exp } \\
\left(\mathrm{KO} \mathrm{e}^{-} \pm 1.5\right)\end{array}$ \\
\hline 4.2 & & 34.0 \\
\hline 26.5 & & 33.3 \\
\hline 21.0 & & 32.3 \\
\hline 27.4 & & 31.5 \\
\hline 32.1 & & 29.6 \\
\hline 37.0 & & 28.3 \\
\hline 40.3 & & 27.1 \\
\hline 45.2 & & 25.2 \\
\hline 50.3 & & 23.0 \\
\hline 56.9 & & 18.8 \\
\hline 61.5 & & 14.6 \\
\hline
\end{tabular}


Table II.B.

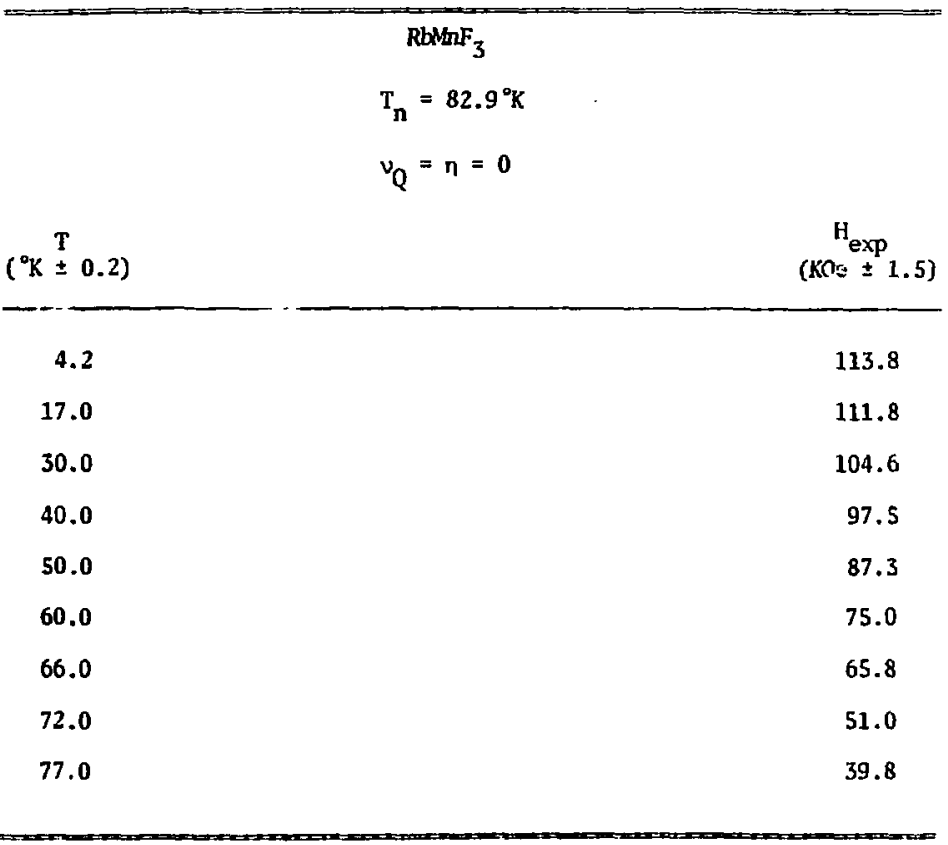


Table III.

\begin{tabular}{|c|c|c|}
\hline Host & $\begin{array}{c}\alpha-\operatorname{MnS} \quad T=156^{\circ} \mathrm{K} \\
\nu_{Q}=\eta=0 \\
\text { Pressure }\end{array}$ & $\mathrm{H}_{\exp }$ \\
\hline$a-M n S$ & 1 bar & $244.4 \pm 2.0$ \\
\hline$a-N \ln S$ & 20 kbar \pm 4 kbar & $253.6 \pm 2.0$ \\
\hline
\end{tabular}


represented to a very good approximation two-dimensional quadratic-1ayer antiferromagnets. Figure 1 shows why this is so. $\mathrm{K}_{2} \mathrm{NiF}_{4}$ is made up of mignetic planes which are the same as those of $\mathrm{KNiF}_{3}$ except that along the $\mathrm{c}$ axis a layer of $\mathrm{KF}$ separates the next magnetic plane, whose spins cancel by symmetry at the central ion site. In performing nuclear Cddoped experiments in these compounds and in $\mathrm{KNiF}_{3}$, the field at the $\mathrm{Cd}$ is generated by four magnetic neighbors of the same sublattice in the twodimensional materials and by six in the three dimensional $\mathrm{KNiF}_{3}$. In the central magnetic plane of $\mathrm{K}_{2} \mathrm{NiF}_{4}$ the bond length $(\mathrm{Ni}-\mathrm{F})$ is $2.003 \mathrm{~A}^{10}$ very close to the Ni-F bond in $\mathrm{NNiF}_{3}, 2.007 \AA .{ }^{10}$ Furthermore, the anisotropic part of the hyperfine structure corstant (A) which measures the unpaired spin density in the $\mathrm{F}^{-}\left(2 \mathrm{p}_{\sigma}\right)$ orbitals has been determined from ${ }^{19} F$ NR and fourd to agree within the experimentel 1 imits $\left(A_{a}\left(K_{N i F}\right)=8.8 \times 10^{-4} \mathrm{~cm}^{-1}\right.$, Ref. $11 ; A_{\sigma}\left(K_{2} \mathrm{NiF}_{4}\right)=9.15 \pm 0.5 \times 10^{-4} \mathrm{~cm}^{-1}$, Ref. 12).

We shall therefore assume that the supertransferred Cd hyperfine field per nearest magne iic neighbor, $H_{0}$, is equal in the two compounds. Using our data for $\mathrm{KNiF}_{3} / \mathrm{K}_{2} \mathrm{NiF}_{4}$ and $\mathrm{RbMF}_{3} / \mathrm{Rb}_{2} \mathrm{MnF}_{4}$ we will now estimate the spin deviation in the two dimensional structures.

Taking $n=2,3$ to define the magnetic dimensionality, we describe the supertransferred hyperfine field at the $c d$ nucleus by

$$
\left.\mathrm{H}_{\mathrm{n}}^{\text {meas }}=z_{\mathrm{n}} \cdot \frac{\left\langle\mathrm{s}_{z}\right\rangle}{\mathrm{s}^{-}}\right)_{\mathrm{n}} \cdot H_{0}
$$

where $Z_{n}$ is the number of magnetic ions contributing to the field and 


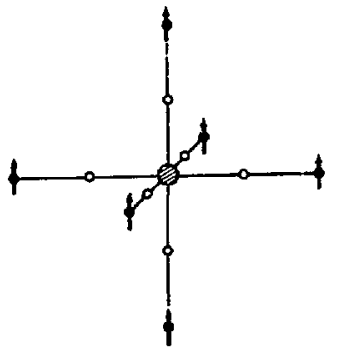

$\mathrm{KNi} \mathrm{F} / \mathrm{Cd}$

- Fluorine

- Ni

- Cd

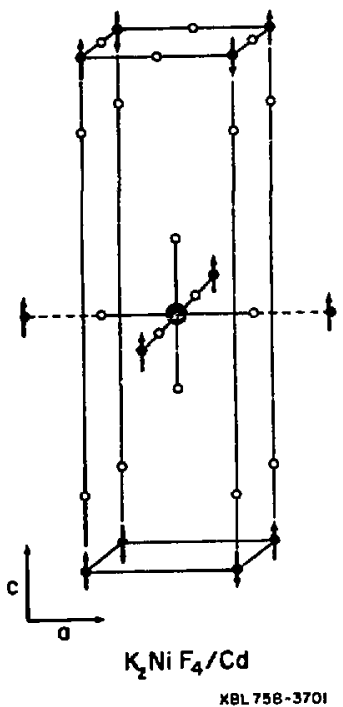

Fig. VI-1. 


$$
\left(\frac{s_{z}}{s}\right)_{n}=1-\frac{\Delta_{n}}{s} .
$$

For $z_{2}=4$ and $z_{3}=6$ it follows that

$$
\Delta_{2}=S\left\{1-\frac{H_{2}^{\text {meas }}}{H_{3}^{\text {meas }}} \cdot \frac{6}{4}\left\{1-\frac{\Delta_{3}}{S}\right)\right\}
$$

In this expression the measured hyperfine fields for the two-dimensional ntiferromagnets $\mathrm{K}_{2} \mathrm{NiF}_{4}$ and $\mathrm{Rb}_{2} \mathrm{MhF}_{4}$ have to be corrected for dipolar fields ${ }^{13}$ of 1.3 and 2.5 kOe respectively.

Al though the zero point spin deviation in a three-dimensional Heisenberg antiferromagnet has not yet been unambiguously resolved, we will assume the value predicted by spin wave theory ${ }^{6}$ (without anisotropy correction) which gives $\Delta_{3}=\mathbf{0 . 0 7 8}$.

Using the experimental field values of Table I (corrected where necessary for dipolar fields) and $\mathrm{S}=1,5 / 2$ for $\mathrm{Ni}^{2+}$ and $\mathrm{Nn}^{2+}$ respectively, we obtain the following estimates from our PAC data

$$
\begin{aligned}
& \Delta_{2}\left(K_{2} \mathrm{NiF}_{4}\right) \sim 0.26 \\
& \Delta_{2}\left(\mathrm{Rb}_{2} \mathrm{MnF}_{4}\right) \sim 0.25
\end{aligned}
$$

Spin-wave formalism yields a theoretical value ${ }^{6}$ for $\Delta_{2}$ of 0.197 (uncorrected for anisotropy). Lines ${ }^{8}$ has calculated the effect of finite anisotropy which can be combined with the anisotropy fields measured by susceptibilities and AFM to give $\Delta_{2}\left(\mathrm{~K}_{2} \mathrm{NiF}_{4}\right)=0.18$ and $\Delta_{2}\left(\mathrm{Rb}_{2} \mathrm{MnF}_{4}\right)$ $=0.167$. These valucs arc in excellent agreement with recent experinental liNiOKR ${ }^{14}$ and $\mathrm{NMR}^{13}$ studies which give $0.20\left(\mathrm{~K}_{2} \mathrm{NiF}_{4}\right)$ and $0.17\left(\mathrm{Rb}_{2} \mathrm{MnF}_{4}\right)$. 
Our estimates of $0.25-0.26$ are high but not unreasonable in view of the fact that Eq. 1 is a rather drastic approximation for describing the supertransferred hyperfine field at the Cd nucleus.

b) Temperature Dependent Sublattice Magnetization

The temperature dependence of the $\mathrm{Cd}$ fields in $\mathrm{RbMFF}_{3}$ and $\mathrm{MnF}_{2}$ is shown in Fig. 2. For comparison we include the MFA Brillouin function of $S=5 / 2$ and the temperature dependence of the sublattice magnetization in $\mathrm{MnF}_{2}$ as measured by ${ }^{19} \mathrm{~F} \mathrm{MR}{ }^{15} \mathrm{RhahF}_{3}$ his the simple perovskite structure of Fig. 1 with six nearest magnetic neighbors which belong to the same sublattice. In $\mathrm{MnF}_{2}$ the field at the impurity has to be corrected for a dipolar contribution ${ }^{15}$ ( 5.8 koe at $4.2 \%$ ) to obtain the supertransferred hyperfine field. For $\mathrm{MnF}_{2}$, the unpaired spin density in the $\mathrm{Cd} s$ shells is caused by two nearest magnetic neighbors of one sublattice and eight next nearest $\mathrm{Mn}^{2+}$ belonging to the other sublattice, with the latter probably dominating. Although the relative sign of the dipolar and hyperfine fields is not known, the dipolar field is at most a $20 \%$ correction and is expected to have the same temperatiure dependence as the hyperfine field. For pure $\mathrm{MnF}_{2}$ the exchange integral of next-nearest-neighbors is much larger than the nearest-nej.ghbor exchange constant, and the replacement of a $\mathrm{Mh}$ ion by the diamagnetic impurity is expected to demagnetize the second nearest neighbors so that the internal field would decrease faster with increasing temperature than the host magnetization. Although the error of the experimentally detemined field in $\mathrm{NnF}_{2}$ is large due to the presence of the quadrupole interaction, the ratio $H(T) / H(0)$ is seen in Fig. 2 to lie systematically below the corresponding ratio for the lost magnetization. 


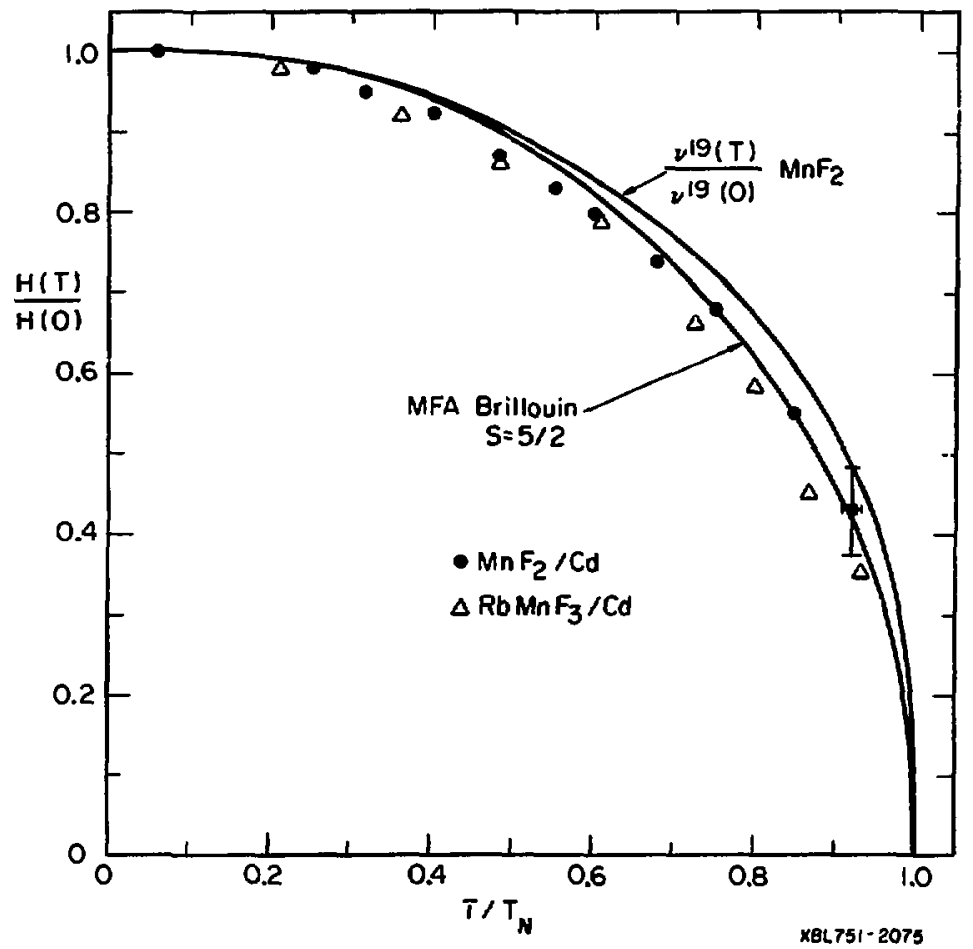

lis. VI-A.2. 
In $\mathrm{RbNhF}_{3}$ the impurity demagnetizes the first nearest magnetic sublattice and the use of a reduced field/reduced temperature plot shows that the effect is comparable to that found in $\mathrm{MnF}_{2}$.

Lovesey and Marshall ${ }^{16}$ have performed numerical calculations of the moment reduction at the first neighbors of a diamagnetic impurity in the molecular field approximation. The calculations were done for face-centered and body-centered lattices and their results for $S=5 / 2$ with either lattice ducrease more rapidly with increasing temperature than our experimental data.

c) Pressure Induced Shift of the Supertransferred Field of $\mathrm{Cd}$ in $\alpha-\mathrm{MhS}_{\mathrm{S}}$ In a magnetic insulator the origin of the field at the doped diamagnetic $C d$ site arises through overlap and covalency effects with transfer of unpaired spin density from the magnetic ions through the intervening ligands and into the s shells of $\mathrm{Cd}$. In an insulator these overlap and covalency effects are short range, and thus the pressure dependence of $H_{h f}(C d)$ relates critically to the bond distances and thus the relative size of the two effects.

In order to estinate the expected shift in $a$-MnS we will use a highly abbreviated model which scales $H_{h f}(C d)$ proportional to $f_{g}\langle y \mid C d n s\rangle^{2}$, where $f_{o}$ is the umpaired spin density transferred from the magnetic ion to the ligand and (L/Ca ns) represents the overlap between the ligand orbitals and the cadrium ns shelis. The ligind orbitals of primary interest would be the $\mathrm{s}^{2-} 3_{\mathrm{p}_{\sigma}}$ wavefunctions, which are unfortunately not available even in free-ion form. Thus we shall make an estimate which uses only the pressure dependence of $f_{a}$, yielding 
$\Delta H_{h f}(C d) / H_{h f}(C d) \cong \Delta f_{\sigma} / f_{\sigma}$. We also assume the kinetic model ${ }^{15}$ for superexchange which connects $\mathrm{J}_{2}$, the interlattice exchange constant, to $f_{0}$ by the relation:

$$
\mathrm{J}_{2} \propto 2 \mathrm{f}_{\pi}^{2}+\mathrm{f}_{\sigma}^{2}+\mathrm{f}_{\mathrm{s}}^{2} .
$$

The subscripts $\pi, \sigma$, and $s$ relate to the nature of the metal-ligand bond. Available data for $\mathrm{MFF}_{2}{ }^{17}$ and $\mathrm{MnO}^{18}$ indicate that $\mathrm{f}_{\pi}$ and $\mathrm{f}_{\sigma}$ are approxinately equal, and that $f_{\pi}^{2}, f_{\sigma}^{2} \gg f_{s}^{2}$. We will assume the same for Mns and replace $J_{2}$ with $3 f^{2}$. Our estimate for $\Delta H_{h f}(\mathrm{Cd}) / H_{h f}(C d)$ is now approximated by $(1 / 2) \Delta \mathrm{J}_{2} / \mathrm{J}_{2}$. One can now use the molecular field approximation, in which $\mathrm{J}_{2}$ is proportional to $\mathrm{T}_{\mathrm{N}}$ and the known ${ }^{19}$ pressure dependence of $\mathrm{T}_{\mathrm{N}}^{-1}\left(\partial \mathrm{T}_{\mathrm{N}} / \partial \mathrm{p}\right)=8 \times 10^{-6}$ bar $^{-1}$ to estimate a relative shift in the hyperfine field of $8 \%$ for $\Delta p=20$ kbar. Thi: represents an upper limit because the corpressibility of MnS will decrease between the Néel point where $\mathrm{T}_{\mathrm{N}}^{-1}\left(\partial \mathrm{T}_{\mathrm{N}} / \partial \mathrm{p}\right)$ is measured and $4.2^{\circ} \mathrm{K}$, where the data were taken. The observed increase in field in Fig. 3 from 244.4 kOe at 1 bar to $253.6 \mathrm{kOe}$ at $20 \pm 4 \mathrm{kbar}$ corresponds to a relative shift of $\Delta H_{h f}(C d) / H_{h f}(C d)$ of $+3.8 \pm 0.8 \%$. Most of the error in the field shift is associated with the pressure calibration, which was accompl ished by measuring the frequency shift of ${ }^{111}$ In in Cd metal and comparing the shift of Raghaven et al. 20

We also tried to perform these pressure experiments on $\mathrm{RhMhF}_{3}$, $\mathrm{KWiF}_{3}$, and $\mathrm{NiO}$ for which we have calculated the $\mathrm{Cd}$-ligand overlap integrals. We found however, that the damping of the spectra for these compounds was both severe and irreversible, and we were unable to extract meaningful ficld shifts. 


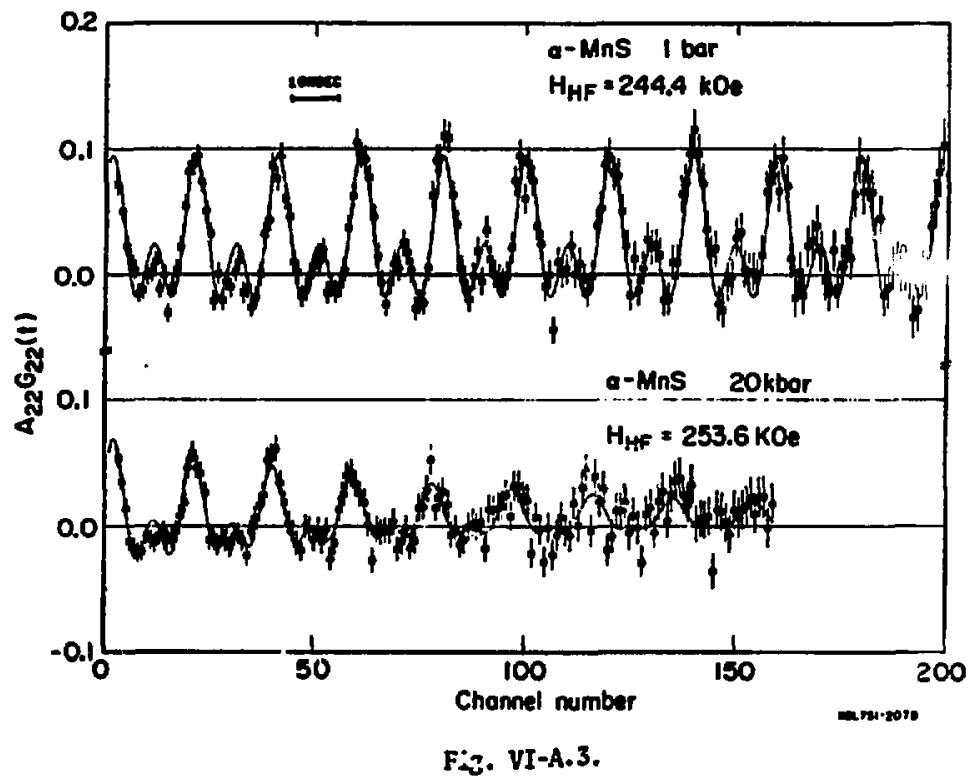




\section{Sumary}

The measurements presented in this paper illustrate the application of PAC methods to the study of solid state phenomena in insulators. We have presented an estimate for the reduction in the spin expectation value due to quantum mechanical zero-point motion which is in fair agreement with MR, susceptibility, and ENDOR results. Additionally we have shown that temperature dependent sublattice magnetizations and pressure induced supertransferred hyperfine field shifts can be measured using the technique of perturbed angular correlations. A wide variety of solid state properties still remain to be explored in the near future. 


\section{ACANOWLEDGEATS}

We thank Duane Newhart for valuable assistance in both the design and construction of the pressure cell. We also wish to acknowledge several helpful discussions with Drs. S. D. Bader, G. Kaindl, and R. Schock. 


\section{REFERENCE:}

1. H. H. Rinneberg and D. A. Shirley Phys. Rev. B 11, 248 (1975).

2. H. H. Rinneberg and D. A. Stirley, Phys. Rev. Lett. 30, 1147 (1973).

3. H. H. Rinneberg, G.P.Schwartz and D. A. Shirley "Companion Paper submitted to Hyperfine Interactions.

4. B. Helber and E.E. Tynan, Rev. Sci. Instrum. 38, 137 (1967).

5. S. D. Bader, Thesis, University of California, Berkeley, January 1974, LBL-2297.

6. P. W. Anderson thys, Rev. 86, 694 (1952).

7. H. I Davis, Phys. Rev. 120, 789 (1960).

8. M. E. Lines, J. Phys. Chem. Solids 31, 101 (1970).

9. J. H. Y. Colpa, E. G. Sieverts, R. H. van der Linde, Physica $\underline{\text { I1, }}$ 573 (1971).

10. Landolt Btrnstein III/7a, Fd. K.-fl. Hellhege, Springer Verlag BerlinHeidelberg-New York, 1973).

11. R. G. Shulman and S. Sugano, Phys. Rev. 130, 506 (1963).

12. E. P. Maarscha11, A. C. Botterman, S. Vega, and A. R. Miedema, Physica 41, 473 (1969).

13. H. W.deWijn, L. R. Walker, and R. E. Halstcdt, Phys. Rev. Bg, 285 (1973).

14. A. H. M. Schrama, Physica 68, 279 (1973).

15. V. Jaccarino in Magnetism V3A, p. 331, Ed. G. R. Rado and H. Suhl, Academic Press, New York, London 1965.

16. S. K. Lovescy and W. Mursha11, Proc. Phys. Soc. 89, 613 (1966).

17. I. Owen and .J. I. M. I7xornley, Repts. Progr. Ihys. 29, 675 (1960). 
18. P. Freund, J. Owen, and B. F. Hahn, J. Hhys. C6, $L 139$ (1973).

19. R. Georges, Acad. Sci. (Paris) 268B 16 (1969).

20. P. Rughavan, R. S. Raghavan, and W. B. Holzapfel, Phys. Rev. Lett. 28, 903 (1972). 


\section{FIGURE CAPTIONS}

Fig. 1. Crystallographic and Magnetic Structure of $\mathrm{NNiF}_{3}$ and $\mathrm{K}_{2} \mathrm{NiF}_{4}$.

Fig. 2. Reduced Temperature/Hyperfine Field Plot for Cd-doped $\mathrm{MnF}_{2}$ and $\mathrm{RbinF}_{3}$.

Fig. 3. Time Spectrum of Cd-doped Ans at $I$ bar and $20 \mathrm{kbar}$. The solid line is a least squares fit to a pure magnetic interaction in polycrystalline material. The time calibration is $0.888 \mathrm{~ns} / \mathrm{channel}$. 
B. Paramagnetic Shifts and Spin-Flop in Supertransferred Hyperfine Structure of ${ }^{\mathrm{Li}} \mathrm{Cd}$ in $\mathrm{RbNhF}_{3}$

Recently [1] we reported the PAC of ${ }^{111} \mathrm{~m} \mathrm{Cd}$ doped as a substitutional impurity into the antiferromagnetic perovskites $\mathrm{RhNhF}_{3}, \mathrm{KCOF}_{3}$ and $\mathrm{KNiF}_{3}$. The hyperfine field at the $c d$ nucleus is caused by unpaired spin density in outer $(45,5 s)$ Cd s-orbitals, transferred from the six nearest magnetic neighbors along linear $\mathrm{Nm}^{2+}-\mathrm{F}^{-}-\mathrm{Cd}^{2+}$ bonds. Since the contact field produced by an s electron is opposite to its spin, the hyperfine field at the Cd nucleus should be parallel to the magnetic moment of the six nearest transition metal ions. In the antiferromagnetic state only the magnitude of the supertransferred hyperfine interaction is obtained, but the sign can be measured in the paramagnetic state by applying an external magnetic ficld.

In this Letter we report the time differential PAC of ${ }^{111} \mathrm{~m} \mathrm{Cd}$ doped into paramagnetic, polycrystall ine $\mathrm{Rb}_{\mathrm{in}} \mathrm{F}_{3}$. The spectra (Fig. 1) were observed at $300^{\circ} \mathrm{K}$ and $87^{\circ} \mathrm{K}$ (close to the Neel point $T_{n}=83^{\circ} \mathrm{K}$ ), with an extermal field $H_{\text {ext }}=31.3 \mathrm{kOe}$ applied perpendicular to the detector axis. In Fig. 1 the perturbation factor $\{2\}, A_{22} G_{22}(\tau)=A_{22}(0.25+0.75$ $\cos \left(4 \pi y_{2} t\right)$ is shown for ${ }^{111 \mathrm{~m}} \mathrm{Cd}$ in $\mathrm{Rb} h \mathrm{~F}_{3}$ and for a diamagnetic standard $\left(\mathrm{CdCl}_{2}\right.$ solution) in the same external field. The higher frequency of ${ }^{1111^{2} \mathrm{Cd}}$ in $\mathrm{RhwhF}_{3}$ corresponds to a higher effective field $\mathrm{H}_{\text {eff }}=\mathrm{H}_{\text {ext }}+\Delta \mathrm{H}$. This paramagnetic shift arises from a supertransferred contact field at the $C d$ nucleus created by polarization of the $\mathrm{N}^{2+}$ electron spin $S=5 / 2$. Since the electronic spin fluctuations are fast compared to the characterist ic $t$ ime of the IMC experiment, only the effect of the time average 


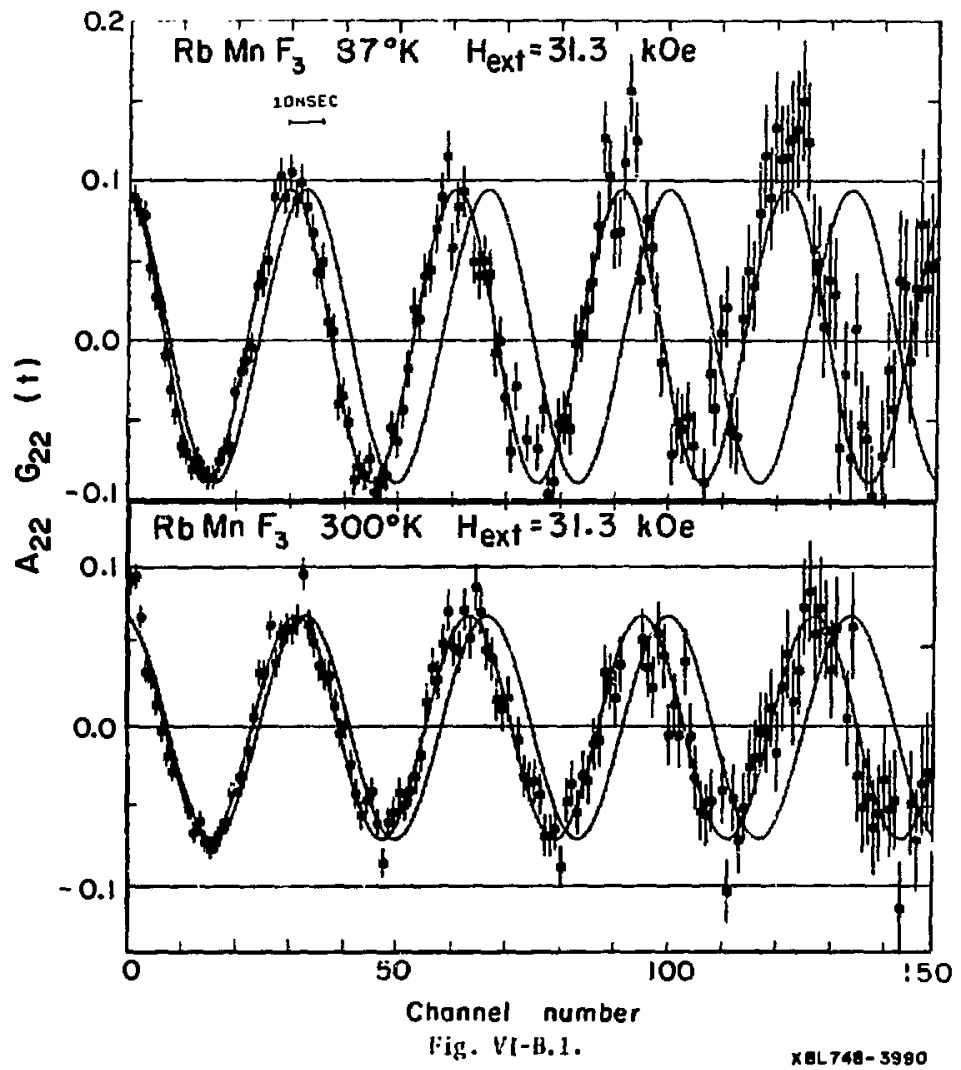


$\left\langle\mathrm{s}_{2}\right\rangle$ is seen. Therefore, $\Delta H=H_{h f^{\prime}}\left(4^{\circ} \mathrm{K}\right)\left\langle\mathrm{S}_{z}\right\rangle / 2.5$, where $\mathrm{H}_{\mathrm{hf}}\left(4^{\circ} \mathrm{K}\right)$ $=116 \mathrm{k}$ ke is taken as the low-tempezature limit of the hyperfine field at the $\mathrm{Cd}$ nucleus in the antiferromagnetic state and $\left\langle s_{z}\right\rangle=5 / 2$ has been used as the local value of $\left\langle\mathrm{s}_{z}\right\rangle$ for $\mathrm{T}=4 \%$. (A small zero-point spin deviation has been neglected in this estimate). The spin expectation value $\left\langle s_{2}\right\rangle=\left(-x_{m}{ }^{H}\right.$ ext $\left.) / g B N_{L}\right)$ in the paramagnetic state can be estimated using the molar susceptibility $x_{m}=\mathrm{C} /(T+\theta)$. In this way we estimate for

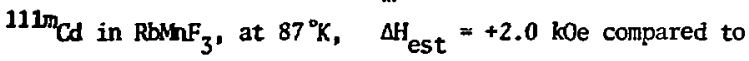
$\Delta H_{\text {obs }}=+3.0 \pm 0.5 \mathrm{k}$ e. The lower susceptibility at room temperature corresponds to a smaller paramagnetic shift (Fig. 1). In this way the following relative shifts $\Delta \mathrm{H} / \mathrm{H}$ ext were observed; $\mathrm{Rb} h \mathrm{~F}_{3}:+9.6 \pm 1.6^{\circ}\left(87^{\circ} \mathrm{K}\right)$, $+5.1 \pm 1.68\left(300^{\circ} \mathrm{K}\right) ; \mathrm{KFeF}_{3}:+5.4 \pm 2.26\left(120^{\circ} \mathrm{K}\right) ; \mathrm{KCOF}_{3}:+4.5 \pm 1.6 \%\left(120^{\circ} \mathrm{K}\right)$ and $\mathrm{KNiF}_{3}:+1.3 \pm 1.66(265 \mathrm{~K})$. Because of the lower susceptibilities, smaller paranagnetic shifts were observed in $\mathrm{KFeF}_{3}, \mathrm{KCOF}_{3}$ and $\mathrm{KNiF}_{3}$ compared to $\mathrm{RbMnF}_{3} / \mathrm{Cd}$. For $\mathrm{KNiF}_{5}$ the large values of $\theta$ and $\mathrm{T}_{\mathrm{N}}$ led to a paramagnetic shift that lay within our experinental error. The experiments reported here are analogous to paranagnetic shift measurements that have been made on F nuclei in these lattices, using MR. Because the $\mathrm{Cd}$ field arises from supertransferred hyperfine structure, these shifts provide a stringent test of the proposed mechanism $[1]$ for spin transfer through $\mathrm{Nn}^{2+}-\mathrm{F}^{-}-\mathrm{Cd}^{2+}$ bonds.

$\mathrm{RbNh}_{3}$ is a well known antiferromagnet with an exceptionally low critical field $H_{c}=2.45 \mathrm{kOe}$ for the spin-flop transition. Above this value the spin axis lies perpendicular to the external field. In polycrystalline $\mathrm{RbmF}_{3} / \mathrm{Cd}\left(4^{\circ} \mathrm{K}\right)$ the hyperfirie fields at the $\mathrm{Cd}$ are randomly oriented for $\mathrm{H}_{\text {ext }}=0$, whereas they are confined in a plane perpendicular 
to the external fieid for $\mathrm{H}_{\text {ext }}>\mathrm{H}_{\mathrm{c}}$. Since PAC is sensitive to the orientation of the hyperfine fields relative to the detector axis, this method lends itself to the observation of the spin-flop transition, as shown (Fig. 2). The time spectra were taken with the detectors at $180^{\circ}$ parallel to $H_{\text {ext }}$. In this case the perturbation factor can be written as $A_{22} G_{22}(t)=A_{22}\left(1-b_{1}-b_{2}+b_{1} \cos \left(2 \pi v_{L} t\right)+b_{2} \cos \left(4 \pi v_{L} t\right)\right)$. The Fourier coefficients $b_{1}, b_{2}$ are equal for the random distribution $b_{1}=b_{2}=0.4$ $\left(\mathrm{H}_{\text {ext }}=0\right)$ and $b_{1}=0, b_{2}=0.75$ if the hyperfine fields are in a plane perpendicular to $\mathrm{H}_{\mathrm{ext}}$. The spin-flop transition was observed as a change in the ratio $b_{1} / b_{2}$ of the Fourier coefficients (Fig. 2). Since a polycrystalline sample was used, the transition is fairly smeared out; for a precise determination of $\mathrm{H}_{\mathrm{c}}$ a single crystal should be used.

This observation gives further microscopic support of both the proposed spin-transfer mechanism and the accepted model for spin-flop transitions. 


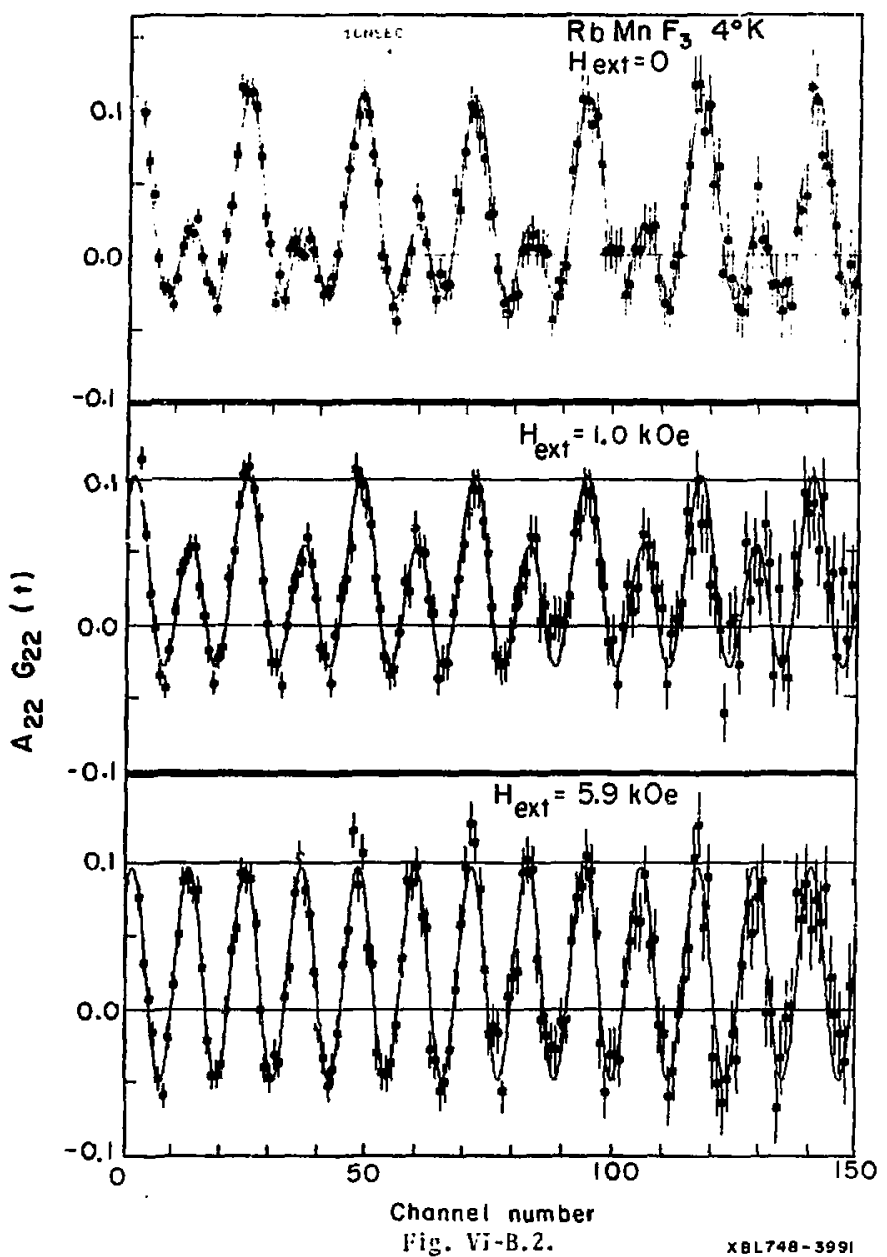


REFERENCES

1. H. H. Rinneberg and D. A. Suirley, Phys. Rev. Letters 30 (1973) 1147.

2. H. Frauenfelder and R. M. Steffen, In Alpha-, Beta-, and Gama-Ray Spectroscopy, ed, by K. Siegbahn (North-Holland, 1965), Vol. 2. 


\section{FIGURE CAPTIONS}

Fig. 1. $\mathrm{PAC}$ of ${ }^{111 \mathrm{~m}} \mathrm{Cd}$ in paranagnetic $\mathrm{RbNhF}_{3}$, compared to a diamagnet ic standard $\left(\mathrm{CdCl}_{2}\right.$ solution) in the same external field $\mathrm{H}_{\text {ext }}=31.3 \mathrm{kOe}$. Fig. 2. Spin-flop transition in $\mathrm{RbMnF}_{3}$ detected by PAC of ${ }^{111 \mathrm{~m}} \mathrm{Cd}$. 
VII. RARE-EARTH VALENCE STATE STUDIES OF THE SERIES RI $n_{3}$ AND RSn $_{3}$ DERIVED FROM QUADRUPOLE COUPLING CONSTANTS

\section{A. Introduction}

Discussions of the electronic structure of metals usually begin by partitioning the lattice into positively-charged ion cores plus itinerant valence $e^{3}$ ectrons. For many metals this separation is a computational shortcut rather than an experimentally observable feature, but in the rare-earth metals and their compounds the "valence state" has a more exact meaning. The $4 \mathbf{f}$ electrons are sufficiently localized to be assigned unambiguously to the ion cores, which then have charges of +3 or sometimes +2 . Considerable theoretical and experimental attention has recently been focussed on the question of non-integral rare-earth valence and valence state changes induced by pressure and temperature. Most of the cases studied to date have been rare-earth chalcogenides which have insulating or semi-metallic properties, but potentially interesting metallic cases have also been known for some time.

A study of the susceptibility ${ }^{1}$ and lattice constants ${ }^{1,2,3,4}$ of the rare-earth series $\mathrm{RIn}_{3}$ indicated that $\mathrm{Yb}$ was in a lower valence state

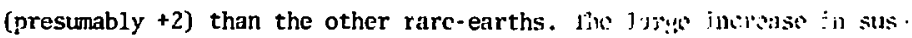
ceptibility ${ }^{1}$ noted on cooling to liquid helium temperatures was taken as an indication that the valence of $\mathrm{Yb}$ had changed from +2 to +3 . In the $\mathrm{RSn}_{3}$ series $\mathrm{EuSn}_{3}$ and $\mathrm{YbSn}_{3}$ both appear with divalent ${ }^{4}$ rare-carths,

*This section of work done in conjunction with D. A. Shirley. 
while $\mathrm{CeSn}_{3}$ is trivalent at room temperature and originally appeared to become quadrivalent $t^{4,21}$ at heliun temperatures. The original ecnclusions concerning the temperature dependence of the rare-earth valencies have been disputed by later Mössbauer ${ }^{5-8}$ studies, tut a consistent picture for the combined In and Sn series has not been published to date.

The $\mathrm{RIn}_{3}$ and $\mathrm{RSn}_{3}$ intermetallics crystallize in the FCC $\mathrm{AuCu}_{3}$ structure, which places the ln or Sn at a site with tetragonal symmetry. The non-cubic nature of this site leads to a non-vanishing electric field gradient $q$ which is highly sensitive to changes in the surrounding rareearth valence state. According to a simple point-charge model, $q$ should scale as $2 / / r^{3}$, where $2 '$ is an effective charge. The functional form of this dependence leads to a great sensitivity advantage of field gradients as opposed to lattice constants for determining valence states. A change from +3 to +2 in rare-earth. valence might increase the lattice constant by $1-3 \%$, but would reduce $q$ in this simple ficture by one-third. We shall show in Section IV that the sensitivity is even greater because q scales as $Z_{R E}-Z_{I n, S n}$ for the $\mathrm{AuCu}_{3}$ structure.

In this paper we report quadrupole coupling constants $\mathrm{e}^{2} \mathrm{qQ} / \mathrm{h}$ determined by time-differential perturbed angular correlation of the $247-\mathrm{keV}$ state of ${ }^{111} \mathrm{Cd}$ following the electron-capture decay of ${ }^{111} \mathrm{In}$. Experimental procedures are described in Section B, and results are given in Section 6. Our results are interpreted in Section D to show that only $\mathrm{YbIn}_{3}$ contains the rare-earth in the divalent state in the $\mathrm{RIn}_{3}$ series, while both $\mathrm{YbSn}_{3}$ and $\mathrm{EuSn}_{3}$ contain divalent rare earths at room temperature. Data taken at $4.2^{\circ} \mathrm{K}$ and in some cases also under $18.5 \mathrm{kbar}$ pressure 
indicate that the divalent state appears to be stable in $\mathrm{YbIn}_{3}$ and $\mathrm{YbSn}_{3}$ and that $\operatorname{CeSn}_{3}$ is non-magnetic at liquid helium temperatures with the Ce in the trivalent state.

B. Experimental

The 2,81-day ${ }^{111}$ In activity, which decays after electron capture via the $173-247 \mathrm{keV}$ cascade of ${ }^{111} \mathrm{Cd}$, was produced by the ${ }^{109} \mathrm{Ag}(\alpha, 2 \mathrm{n})$ reac$t$ ion induced by $\alpha$-particle bombardment of a $0.005 \mathrm{~cm}$ natural $\mathrm{Ag}$ foil at the Lawrence Berkeley Laboratory 88-Inch Cyclotron. The use of natural $\mathrm{Ag}\left(51 \% \mathrm{Ag}{ }^{107}, 49^{\circ} \mathrm{Ag}^{109}\right)$ Ilecessitated waiting about 36 hours for the $\mathrm{In}^{109}(4.3 \mathrm{hr})$ to decay away, after which the $\mathrm{In}$ and $\mathrm{Ag}$ were separated using magnesiun hydroxide precipitation at a $y$ l of $8-9$. Generally $.1-.2$ $\mathrm{mg}$ of In carrier was added, and the activity plated out of a formate-buffered sulfate solution (pH 2) onto an In or Sn foil.

The active foil and stoichionetric amounts of rare-earth metals were then arc-melted under argon on a water cooled cu hearth. Because the components for $\mathrm{YbIn}_{3}, \mathrm{YbSn}_{3}$, and $\mathrm{EuSn}_{3}$ reacted vigorously during melting, some caution was required to produce these compounds without splattering and loss of material. An additional check we also made these compounds by encapsulating the pure metals in a tantalum cruicible and sealing under argon in a quartz tube prior to oven heating. Samples made by these two methods yielded identical coupling constants within experimentai error.

Efforts to prepare $\mathrm{EuIn}_{3}$ failed. This is consistent with refer-

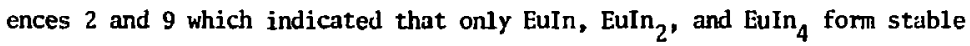


intermetallics. The attempt to make $\mathrm{EuIn}_{3}$ resulted in a spectrum which was identical to that of EuIn 4 when the latter compound was made stoichiometrically. The heavy $\mathrm{RSn}_{3}$ compounds from $\mathrm{Tb}$ to $\mathrm{I}: \mathrm{u}$ (with the exception of $\mathrm{YbSn}_{3}$ ) cannot be made in the FCC phase in an arc-furnace or oven at conventional pressures. Our samples of $\mathrm{GdSn}_{3}$ also showed evidence for not being single phase, but there was no difficulty in extracti..g the quadrupole coupling constant to $\pm 10^{\circ}$.

The time spectra were recorded on four $3.8 \times 5.1 \mathrm{~cm} \mathrm{NaI}$ (T1) scintillation crystals mounted on 56 DVP photomultipliers using conventional fastslow logic. The detectors were arranged in a square array, labeled cyclically A,B,C,D. Two $180^{\circ}$ ( $A C$ and $B D$ ) and two $90^{\circ}$ ( $3 C$ and $A D$ ) coincidence spectra were routed to a Scip, 1000 channel pulse-height analyzer and later dumped onto magnstic tape for subsequent analysis. The time resolution measured with a ${ }^{22} \mathrm{Na}$ source with the energy windows set at 173 and $247 \mathrm{keV}$ was 2.0 ns FWM. The time-to-amplitude converter (Ortec 437) was calibrated using an Eldorado 610 digital delay generator.

then four detectors are used in this way, with A and B the "start" detectors (rccording the 173-keV photons) and with C and D the "stop" detectors (247-keV photons), the time-modulation function $\Phi_{22} A_{22} G_{22}(t)$ can readily be extracted ${ }^{10}$ from the four coincidence counting rates

$$
H_{A C}(\theta, t)=N_{0} e^{-t / \tau} N \varepsilon_{A C}\left[l+Q_{22}(A C) A_{22} G_{22}(t) P_{2}\left(\cos \theta_{A C}\right) l,\right.
$$

etc., by forming the ratio

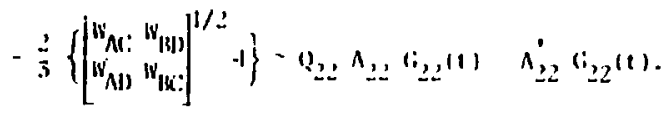


This eliminates the total counting rate $N_{0}$, the nuclear lifetime $\tau_{N}$, and to a good approximation the coincidence efficiencier. $\varepsilon_{\mathrm{AC}}$, etc. Only an average solid-angle correction factor $\bar{Q}_{22} \cong 0.5$ remains. For convenience it is absorbed into the "effective" angular correlation ccefîizient $A_{22}$.

\section{Results}

The In and Sn sites in these compounds have tetragonal symmetry. They can therefore be treated as cases of randomly-oriented, axiallysymmetric electric field gradients. The perturbation factor for this case, with $I=5 / 2$, has the anaiytic form ${ }^{11}$

(1) $G_{22}(t)=1 / 5+(13 / 35) \cos \omega_{0} t+(2 / 7) \cos 2 \omega_{0} t+(1 / 7) \cos 3 \omega_{0} t$. Here $\omega_{0}$ is a basiz frequency given by

(2) $\omega_{0}=3 \mathrm{e}^{2} q Q / 20 \mathrm{~h}$.

Figures 1 and 2 show the time modulation of typical $A_{22}^{\prime} G_{22}(t)$ func-ions due to the frecession of the quadrupole moment of the $24 /-\mathrm{keV}$ level of ${ }^{111}$ Cd in the electric field gradient present at the In and Sn sites. The solid curve is a least-squares fit to Eq. 1. The fit yields the basic radial medulation frequency $\omega_{0}$ where $q$ is the electric field gradient for $\mathrm{Cd}$ at the In site and $Q$ is the quadrupole moment of the $247-\mathrm{keV}{ }^{111} \mathrm{Cd}$ level In Table I we have listed the lattice constants as well as the quadrupole coupling constant $\mathrm{e}^{2} \mathrm{qQ} / \mathrm{h}(\mathrm{M} / \mathrm{z})$, derived using Eq. (2). 


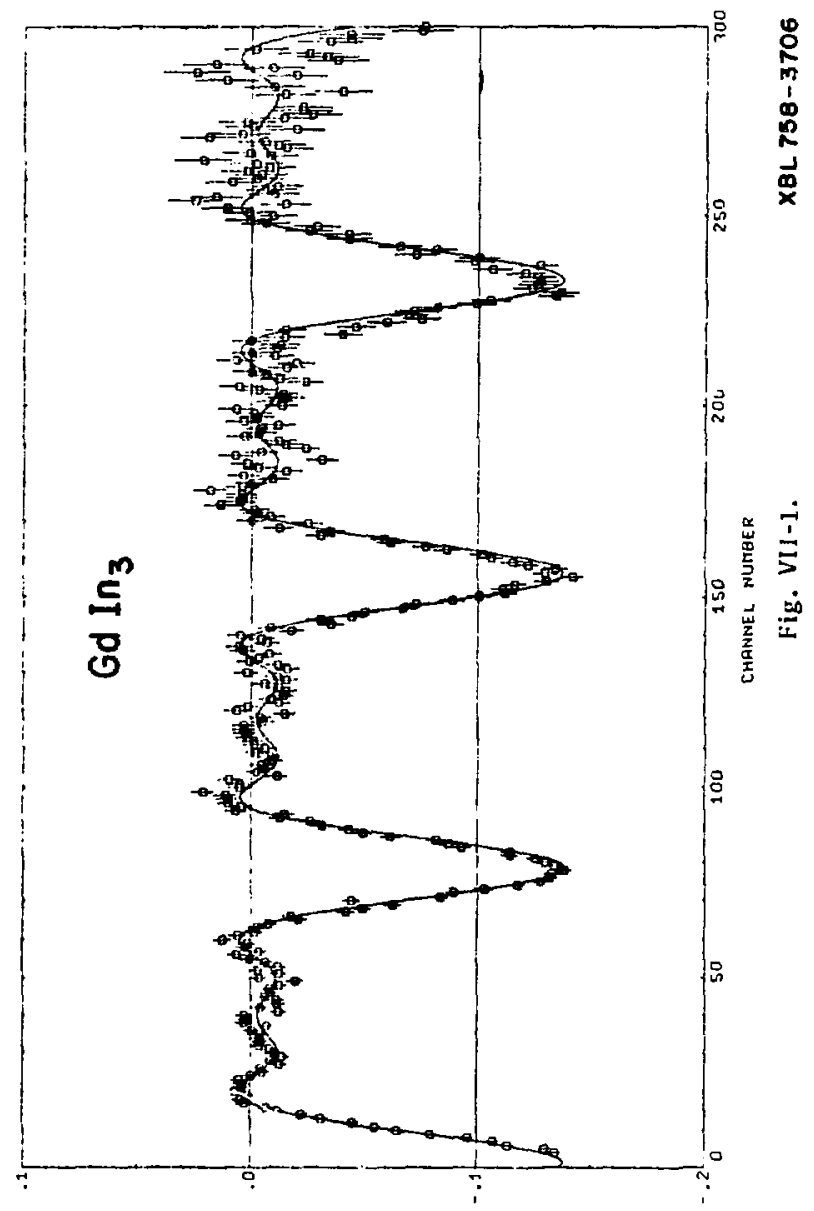

$(t)^{2 z_{9}}{ }^{2 z_{V}}$ 
$-100-$

\section{$\mathrm{Ce} \mathrm{Sn}_{3}$}
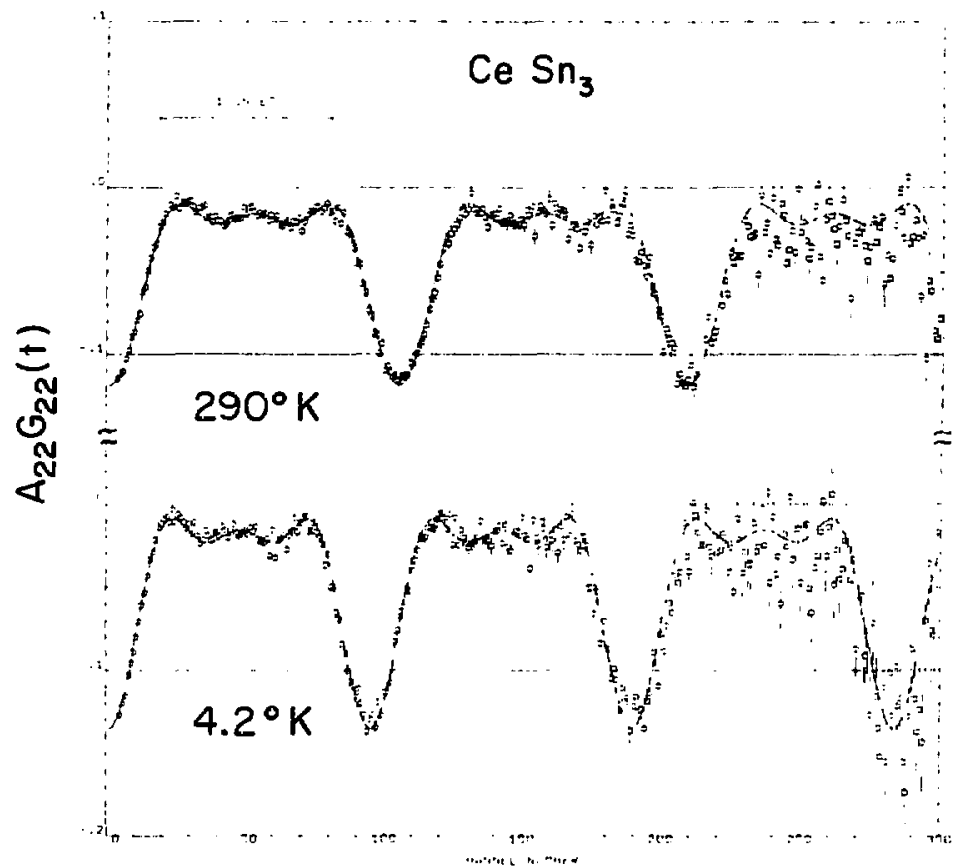

X8L 758-3705

Fig. V1J.2. 
Tabie I.A

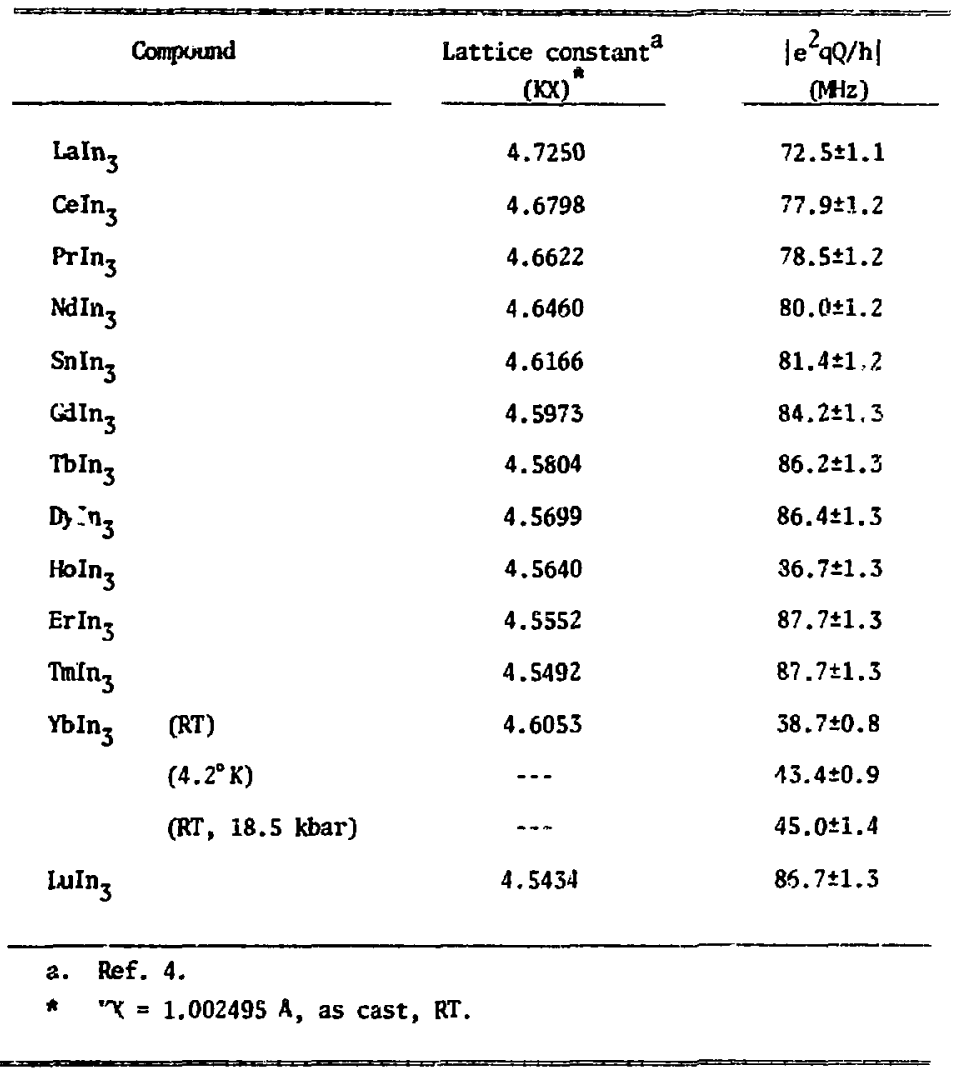


Table I.B

Cormpound

$\begin{array}{ll}\mathrm{Sn}_{3} & (\mathrm{RT}) \\ & \left(4.2^{\circ} \mathrm{K}\right)\end{array}$

-

$\mathrm{Sn}_{3} \quad$ (RT)

(RT, 18.5 kbar)

(4. $\left.2^{\circ} \mathrm{K}\right)$

$\left(4.2^{\circ} \mathrm{K}, 18.5 \mathrm{kbar}\right)$

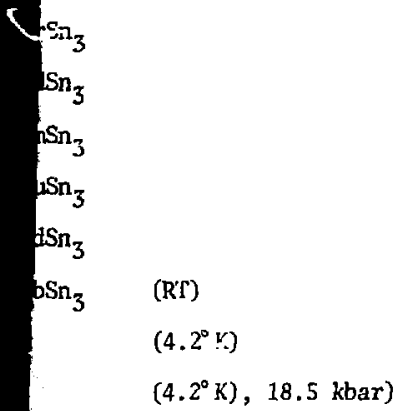

Lattice constant ${ }^{a}$

$(\mathrm{KX})^{*}$

4.7598

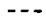

4.7119

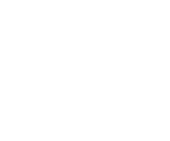

4.7064

4.6965

4.6772

4.7349

4.6681

4.6720

$21.9 \pm 1.1$

$25.6 \pm 1.3$

$29.7 \pm 1.8$

Ref. 4.

$\mathrm{KX}=1.002495 \AA$, as cast, $\mathrm{RT}$ 


\section{Interpretation}

Recent theoretical discussions ${ }^{12-16}$ have highlighted the difficulties of calculating the valence electron contribution to the electric field gradients (EFG) of metals and alloys when the exact charge distribution around the atoms is not known. The essential difficulty arises because the charge distribution of the itinerant valence electrons is actually largely centered around the ion cores, where it tends to shield the innic core contribution to $q$, and can result in either the core or valence electrons dominating and determining the sign of $q$. Even the separation of the EFG into separate independent core and electron contributions has been brought into questiun by a recent study ${ }^{17}$ of the systematics of experimental data on metal and alloy gradients which indicates an apparent scaling of the electron cortribution to the total ionic core term eq 1 at $\left(1-\gamma_{\infty}\right)$.

We will not attempt a quantitative interpretation of the magnitude of $\mathrm{e}^{2} \mathrm{qQ} / \mathrm{h}$, which would require a rather elaborate calculation of dubious value in light of the present understanding of the contributions to electric field gradients in metals and alloys. Instead we will adopt the form

$$
\text { eq }=e_{1 \text { at }}\left(1-\gamma_{\infty}\right)\left(1+F_{e}\right)
$$

suggested by Ref. 18 and empirically justified on a systematic basis by Raghavan et. al. ${ }^{17}$ In Eq. 1 eq $_{1 \text { at }}$ is the point charge contribution of the "ionic" cores, $\gamma_{\infty}$ the Sternheiner factor, and $\mathrm{F}_{\mathrm{e}}$ a term which reflects the valence electron contribution. 
Using the method of plane-wise sumation ${ }^{19}$ for a lattice of infinite extent, de Wette and Schacier ${ }^{20}$ performed the iattice sum calcuiation for tr $\equiv$ FCC structure at the In or Sn site. The result of their calculation yields

$$
\mathrm{eq}_{1 \mathrm{at}}(\mathrm{In}, \mathrm{Sn})=-\mathrm{e}(8.67)\left(\mathrm{Z}_{\mathrm{RE}} \mathrm{z}_{\mathrm{In}, \mathrm{Sn}}\right) / \mathrm{a}_{\mathrm{o}}^{3},
$$

where $z_{R E}$ and $z_{I n, S n}$ are the assumed metal valencies and $a_{n}$ is the lattice constant. The factor $\mathbf{8 . 6 7}$ for the infinite lattice can be compared to the number 7.0, which we calculated assuming only interactions out to the third nesrest neighbor. Most of the contribution to the EFG is thus short-range in rature. The quadrupole coupling constants of Table I are related to Eq. 3 by

$$
\left|\mathrm{e}^{2} \mathrm{qQ} / \mathrm{h}\right|=\left|\mathrm{e}^{2} \mathrm{Q}(8.67)\left(\mathrm{R}_{\mathrm{RE}} \mathrm{Z}^{-\mathrm{Z}} \mathrm{In}, \mathrm{Sn}\right)\left(1-\gamma_{\infty}\right)\left(1+\mathrm{F}_{\mathrm{e}}\right) / \mathrm{ha}_{\mathrm{O}}^{3}\right|
$$

The study of the rare earth valence and its possible fluctuations are contained in the form of Eq. 5. If the rare earths in the series $\mathrm{RIn}_{3}$ or $\mathrm{RSn}_{3}$ were all in the +3 valence state and the electronic screening was constant, then a plot of $\mathrm{e}^{2} \mathrm{qQ} / \mathrm{h}$ vs $z^{1} / \mathrm{a}_{0}^{3}$ would be roughly constant. Any slow variation in screening throughout the series should be reflected in $q$, while a different valence state should appear as an abrupt change. To factor out the $a_{0}^{-3}$ effect due to the gradual variation in lattice constants, we have plotted $\left(a_{0}^{3}\right)\left(e^{2} q Q / h\right)$ vs atomic number. Figures 3 and 4 show the data for the $\mathrm{RIn}_{3}$ and $\mathrm{RSn}_{3}$ compounds with $\mathrm{AuCu}_{3}$ structure as well as the lattice constants of $\operatorname{Ref}$. 4. The near constancy of $\left(a_{0}^{3}\right)\left(e^{2} q Q / h\right)$ throughout the series argues strongly for the rare carth ions being in the same valence state-presumably +3- and for the screening effect of the valence 


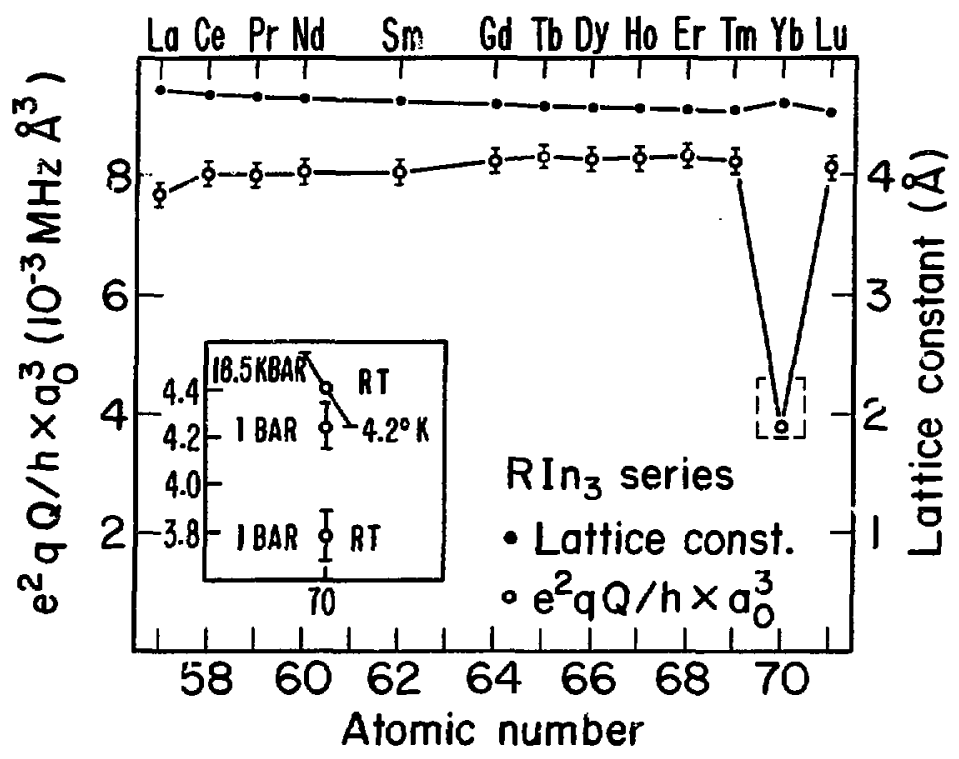

Fig. VII-3. 


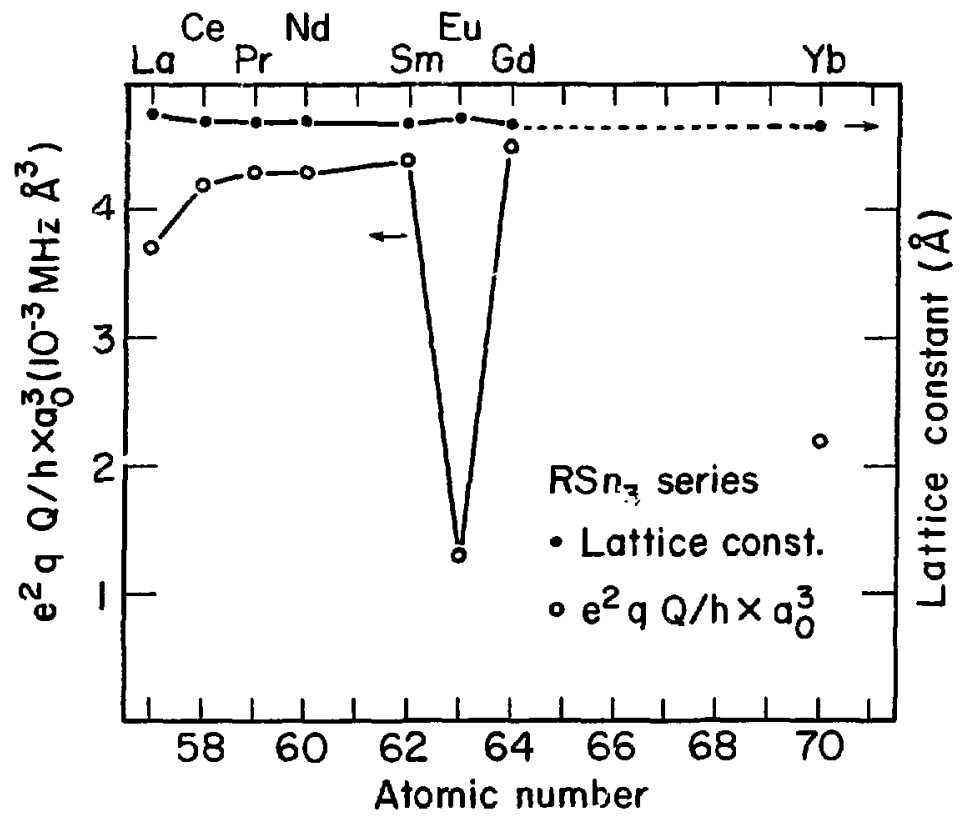

Fig. VII-4. 
electrons being essentially the same across the rare earth row in these compounds. Dramatic drops are seen to occur at $\mathrm{YbIn}_{3}, \mathrm{EuSn}_{3}$, and $\mathrm{YbSn}_{3}$. The lattice constant variations for these three compounds from the +3 line are of the order $1-2 \%$, and clearly cannot account for the magnitude of the reducticos. The percentage deviation in the quadrupole coupling constants is $~ 30-50$ times larger than that noted in the lattice constants, confirming the great sensitivity of this parameter to the rare-earth valence state.

Room temperature susceptibilities, ${ }^{1,21}$ lattice constants, ${ }^{1-4}$ and Mossbauer spectra ${ }^{5-8}$ are all consistent with the assignm ant of the divalent state to the rare earth in these three compounds at room temperature. Gschneidner $^{22}$ has also chermodynamically evaluaied heat of formation data for a number of $\mathrm{Yb}$ and Eu compounds and has estimated that for $\mathrm{Yb}$ or $\mathrm{Eu}$ to be trivalent in a partic'slar compound requires the heats of formation for the trivalent lanthanides to be more negative than roughly -9 and $-23 \mathrm{kcal} /$ gm- at of rare earth respectively. One thus generally expects :.b and especially Eu to be divalent in all but the most ionic or covalent compounds. We would like to show that the assignment of $\mathrm{di}$ - and trivalent states is also consistent with the absolute magnitudes of the quadrupole coupling constants. Equation 5 requires the lattice constants, and we will adopt the value $a_{0}=4.65 \mathrm{~A}$ for the purpose of estimating the coupling constants. The error il using an average lattice constant is less than the uncertainty in the quadrupole monent $Q$ of the $I=5 / 2$ intermediate state, whose literature values span a factor of three. We will use the value $Q=0.44 b^{18}$ and the Stemheimer factor for $\mathrm{Cd}^{2+}$ calculated by Feiock and Johnson ${ }^{23} \gamma_{\infty}=-29.27$. 
The choice of the charges at the In and Sn sites is dictated by several considerations. In the $\mathrm{RIn}_{3}$ series the assignment of +3 for $\mathrm{In}$ and +3 for the rare earths (except for $\mathrm{Yb}$ ) would cause the electric field gradient to vanisk identically. This is not an artifact of performing a finite lattice sum but is a rigorous symmetry argument for an FCC lattice in which all sites are equivalent1y charged. From Mössbauer measurements in EuSn 3 , Loewenhaupt and flïfner ${ }^{8}$ assign $S n$ as divalent rather than tetravalent. This pulling of change toward In or $\mathrm{Sn}$ is consistent with the electronegativities tabulated by Teatum ${ }^{21}$ et al., who assign values around 1.2 to the rare earths and 1.4-1.6 for In and Sn. Finally one requires that the order of magnitude of the coupling constants should be predicted reasonably regardless of whether the rare earths are di- or trivalent. If one assigns +3 to the rare earths, then a $r 1$ valence at $I n$ and +2 at $S n$ is consistent with these considerations. We will also take $\left|\left(1+F_{e}\right)\right| \approx 1$ which is consistent with the observations of Ref. 17 that $F_{e}$ has values of order -2 to -3 . It would be perhaps more realistic to take $\left|Q\left(1+F_{e}\right)\right| \approx 0.5$ since the uncertainty in $Q$ is so large. The assignument of +1 to In and +2 to $\mathrm{Sn}$ deserves an additional conment. These valence assignments would imply neutral $\mathrm{Cd}\left(4 \mathrm{~d}^{10} 5 \mathrm{~s}^{2}\right)$ rather than $\mathrm{Cd}^{2+}$ $\left(4 \mathrm{~d}^{10}\right)$ with the associated change in Sternheimer factor. One can estimate this effect by looking at the configurations for $i$ in $+7\left(4 d^{10}\right)$ and +5 $\left(4 \mathrm{~d}^{10} \mathrm{~s}^{2}\right)$ valence states, for which the Stemheimer factors ${ }^{23}$ are $Y_{\infty}\left(I^{+7}\right)=-16.84$ and $Y_{\infty}\left(I^{+5}\right)=-18.24$. This effe $=t$ is of order $10^{\circ}$, yielding $\gamma_{\infty}\left(\mathrm{Cd}^{0}\right) \sim-32$, and can be incorporated into $\left|\mathrm{Q}\left(1-\gamma_{\infty}\right)(1+\mathrm{Fe})\right|$. For $Q=0.44$ barns, $1-Y_{\infty} \simeq-33$ and $\left|1+F_{e}\right| \sim 1$, we abtain $\left|Q\left(1-\gamma_{\infty}\right)\left(1+F_{2}\right)\right|$ - 14.5, with a large tacertininty. Using this estimate and $a_{0}=4.65 \AA$, we obtiin 


$$
\left|\mathrm{e}^{2} \mathrm{qQ} / \mathrm{h}\right|=44\left(\mathrm{z}_{\mathrm{RE}}-z_{\mathrm{In}, \mathrm{Sn}}\right) \mathrm{MHz} .
$$

Considering first the $\mathrm{RIn}_{3}$ compounds with $Z_{\mathrm{RE}}=+3$ and $\mathrm{Z}_{\mathrm{In}}=+1$, we expect a coupling constant of order $88 \mathrm{M} / 2$. Most of the experimental values are between 80 and $90 \mathrm{M} / \mathrm{z}$. The agreement is certainly fortuitous in view of the uncertainties in $Q, F_{e}$, and the valence assignment of +1 for In. However, a rather consistent picture follows from these assignments. For ibIn 3 with a divalent rare earth, one expects a $50 \%$ reduction to $44 \mathrm{MHz}$. The measured value is $38.7 \mathrm{MHz}$. In addition, one expects that if $\mathrm{Sn}$ is divalent the Sn series with trivalent rare earths should be around $44 \mathrm{MHz}$, and the experimental values generally fall between $40-45 \mathrm{MHz}$. For ${ }^{2} \mathrm{Sn}^{x+2}$ however, the divalent rare ear'hs $\mathrm{EuSn}_{3}$ and $\mathrm{YbS}_{3}$ should have coupling constants equal to zero by symetry. The EuSn$n_{3}$ value has dropped to 12.7 $M \mathrm{z}$, while $\mathrm{YbSn}_{3}$ is higher at $21.9 \mathrm{Mz}$. The failure of these values to vanish identically points out the deficiency of expecting more than qualitative agreement with our estimates of the coupling constants.

As an additional check we measured $\mathrm{BaSn}_{3}$, in which one expects tie stability of the Xe core to insure Ba being divalent, and found a coupling constant of approximately $19 \mathrm{MHz}$. The purpose of the estimate was not to justify a point charge model or our assignment of the charges to the rare earths, In, and $\mathrm{S} n$, but rather to emphasize that a rather simple picture of the field gradient can yield useful insight into the qualitative trends in these alloys.

We now examine the question of the temperature and pressure dependence of valence fluctuations in these alloys. Susceptibility ${ }^{1,21}$ data, thermal expansion measurements, ${ }^{4}$ and lattice constants ${ }^{4}$ had indicated 
that $\mathrm{YbI}_{3}$ and $\mathrm{CeSn}_{3}$ appeared to become trivalent and partially tetravalent respectively on cooling to $4.2^{\circ} \mathrm{K}$ and that $\mathrm{YbSn}_{3}$ might exist in a mixed valence state at room temperature. We have measured all of these compounds at $4.2^{\circ} \mathrm{K}$ and find shifts of order $10-15 \%$, which are not nearly large enough to be representative of complete change in valence state. We feel that most of the shift can be accountrs? for by the thermal contraction of the Jattice. Figure 2 also shows that $\mathrm{CeSn}_{3}$ is .zon-magnetic. in accord with earlier Mossbauer ${ }^{5}$ data. It is interesting to note that $\mathrm{YbSn}_{3}$ probably undergoes a lattice distortion on cooling to liquid helium temperatures, because ${ }^{\circ}$ ? PAC pattern is clearly distorted from that expected for axial symmetry. We have checked this to see if it was an artifact of the sample preparation and have found it to be a reproducible feature of the low temperature spectra. Room temperature spectra show no such distortion.

$\mathrm{YbIn}_{3}(\mathrm{RT}), \mathrm{BSSn}_{3}(4.2 \mathrm{~K})$, and $\operatorname{CeSn}_{3}(\mathrm{RT}$ and $4.2 \mathrm{~K}$ ) were also $\mathrm{rm}$ in a clamping type pressure cell to $18.5 \mathrm{kbar}$ in order to see if we cotld induce a valency fluctuation. Shifts on the order of those associated with cooling to helium temperatures were observed, but in no case did we observe an increase which would put the points on the "trivalent line" of Figs. 3 and 4. We conclude that the compounds $\mathrm{CsSn}_{3}, \mathrm{YbSn}_{3}$, and $\mathrm{YbIn}_{3}$ show no evidence for a bulk valence instability down to liquid helium temperatures and pressures to 18.5 kbar. Previous A6ssbauer data on $\operatorname{Cosn}_{3} 5$ and $\mathrm{VbSn}_{3}{ }^{7}$ have come to similar conclusions.

In sumary we have systematically studied the $\mathrm{RIn}_{3}$ and $\mathrm{RSn}_{3}$ compounds with $\mathrm{AuCu}_{3}$ struteture using TDPAC and have demonstrated the high 
sensitivity of this technique to the rare earth valence state. We have also conducted experiments to probe the possible temperature and pressure dependence of the rare earth valence in $\mathrm{CeSn}_{3}, \mathrm{YbSn}_{3}$, and $\mathrm{YbIn}_{3}$ and fird ro evidence for bulk valence fluctuations in these compounds. 


\section{ACXNOWLEDGMENS}

One of us (GPS) wishes to thank Ruth Mary Larimer and Harry Harrington for assistance with the 88-Inch Cyclotron target and Wini Heppler and Gerda Bolz for help in working up the activity and sample preparation. 


\section{REFEREICES}

1. K. H. J. Buschow, H. W. De Wijn, and A. M. Van Diepen J. Chen. Phys. 50(1) 137 (1969).

2. Yu. B. Kuz'ma and V. Ya., Markiv Kristallografiya 9 (2) 279 (1964).

3. J. L. Moriarity, J. E. Humphreys, R. O. Gordon, and N. C. Baenziger Acta Crystallog. 21(5), 840 (1966).

4. I. R. Harris and G. V. Raynor, J. Less Common Metals 9, 7 (1965).

5. G. K. Shenoy, B. D. Dumlap, and G. M. Kalvius, J. App1. Phys. 41, 1317 (1970).

6. F. Borsa, R. G. Barnes, and R. A. Reese, Phys. Stat. So1. 19, 359 1967).

7. A. Percheron-Guegan, J.-C. Achard, O. Gorochou, F. Gonzalez-Jimenez, and P. Imbert, J. Less Common Metals 37, 1 (1974).

8. M. Loewenhaupt and S. Hufner, Physics Letters 30A, 309 (1969).

9. W. Koester and J. Meixner, 2. Metal1k. 56, 695 (1965).

10. B. Erik Karlsson, Arkiv. Fysik 22, 1 (1962).

11. R. M. Steffen and H. Fravenfelder, Perturbed Angular Correlations, ed. E. Karlsson, E. Matthias, and K. Siegbahn (North Holland, 1964).

12. R. E. Watson, A. C. Cossard, and Y. Yafet, Phys. Rev. 140, A375 (1965).

13. N. C. Mohapatra, C. M. Singal, and T. P. Mas, Phys. Rev. Lett. 31, 530 (1973).

14. N. C. Mohapatra, C. M. Singal, T, P. Das, and P. Jena, Phys. Rev, Lett. 29, 456 (1972). 
15. K. W. Lodge and C. A. Scholl, J. Phys. F: Metal Fhys. 4, 2073 (1974).

16. K. C. Das and D. K. Ray, Phys, Rev. 187, 777 (1969).

17. R. S. Raghavan, E. N. Kaufmann, and P. Raghavan, Phys. Rev. Lett. 34,1280 (1975).

18. E. Botenstedt, U. Ortabasi, and W. H. E1lis, Phys. Rev. B $\underline{6}$, 2909 (197<).

19. F. W. De Wette, Phys. Rev. 123, 103 (1961).

20. F. W. De Wette and G. E. Schacher, Phys. Rev. 137, A92 (1965).

21. T. Tsuchida and W. E. Wallace, J. Chem. Phys. 43, 3811 (i965),

22. K. A. Gschneidner, Jr., J. Less-Conmon Metals 17, 1 (1969).

23. F. D. Feiock and W. R. Johnson, Phys. Rev. 187, 39 (1969).

24. E. Teatum, K. Gschneidner, Jr., and J. Waber, LA-2345 (1960). 


\section{FIGURE CAPTIONS}

Figure 1. Experimental and least squares fit (solid line) time moculation spectrum for ${ }^{111_{I n}}$ in $\mathrm{GdIn}_{3}$. The time calibration is $1.03 \mathrm{~ns} / \mathrm{channel}$.

Figure 2. Experimental and least squares fit (solid line) time modulation spectra of 111 In in CeSnz at 290 and $4.2^{\circ} \mathrm{K}$. The time calibration is $1.62 \mathrm{~ns} / \mathrm{channe1.}$

Figure 3. Lattice constants and quadrupole coupling data for $\mathrm{RIn}_{3}$ compounds plotted against atomic number.

Figure 4. Lattice constants and quadrupole coupling dat. for $\mathrm{RSn}_{3}$ compounds plotted against atoric number. 